British Journal of Mathematics \& Computer Science

13(6): 1-54, 2016, Article no.BJMCS.19235

ISSN : 2231-0851

SCIENCEDOMAIN international

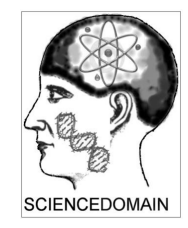

\title{
Distributional SAdS BH Spacetime-Induced Vacuum
} Dominance

\author{
Jaykov Foukzon ${ }^{1 *}$, A. Potapov ${ }^{2}$ and E. Men'kova ${ }^{3}$ \\ ${ }^{1}$ Center for Mathematical Sciences, Israel Institute of Technology, Haifa, 3200003, Israel. \\ ${ }^{2}$ Kotel'nikov Institute of Radioengineering and Electronics, Russian Academy of Sciences, \\ Moscow, 125009, Russia. \\ ${ }^{3}$ All-Russian Research Institute for Optical and Physical Measurements, Moscow, 119361, Russia.
}

Article Information DOI: 10.9734 BJMCS/2016/19235 Editor(s):

(1) Yang-Hui He, Professor of Mathematics, City University London, UK and Chang-Jiang Chair Professor in Physics and Qian-Ren Scholar, Nan Kai University, China; Tutor and Quondam-Socius in Mathematics, Merton College, University of Oxford, UK. (2) David F. Mota, Institute of Theoretical Astrophysics, University of Oslo, Norway. (3) Morteza Seddighin, Indiana University East Richmond, USA. Reviewers:

(1) Jos Antonio de Freitas Pacheco, University of Nice-Sophia Antipolis, France. (2) Francisco Bulnes, Department in Mathematics and Engineering, Tecnolgico de Estudios Superiores de Chalco, Mexico. Complete Peer review History: http://sciencedomain.org/review-history/12977

Original Research Article

Received: $30^{\text {th }}$ May 2015

Accepted: $10^{\text {th }}$ July 2015

Published: $15^{\text {th }}$ January 2016

\begin{abstract}
This paper dealing with extension of the Einstein field equations using apparatus of contemporary generalization of the classical Lorentzian geometry named in literature Colombeau distributional geometry, see for example [1], [2], [3], [4], [5], [6], [7] and [32]. The regularizations of singularities presented in some solutions of the Einstein equations is an important part of this approach. Any singularities present in some solutions of the Einstein equations recognized only in the sense of Colombeau generalized functions [1], [2] and not classically. In this paper essentially new class Colombeau solutions to Einstein field equations is obtained. We leave the neighborhood of the singularity at the origin and turn to the singularity at the horizon. Using nonlinear distributional geometry and Colombeau generalized functions it seems possible to show that the horizon singularity is not only a coordinate singularity without leaving Schwarzschild coordinates. However the Tolman formula for the total energy $E_{T}$ of a static and asymptotically flat spacetime, gives $E_{T}=m$, as it should be. The vacuum energy density of free scalar quantum field $\Phi$ with a
\end{abstract}

${ }^{*}$ Corresponding author: E-mail: jaykovfoukzon@list.ru; 
distributional background spacetime also is considered. It has been widely believed that, except in very extreme situations, the influence of gravity on quantum fields should amount to just small, sub-dominant contributions. Here we argue that this belief is false by showing that there exist well-behaved spacetime evolutions where the vacuum energy density of free quantum fields is forced, by the very same background distributional spacetime such distributional BHs, to become dominant over any classical energy density component. This semiclassical gravity effect finds its roots in the singular behavior of quantum fields on curved spacetimes. In particular we obtain that the vacuum fluctuations $\left\langle\Phi^{2}\right\rangle$ have a singular behavior on BHs horizon $r_{+}:\left\langle\Phi^{2}(r)\right\rangle^{\sim}\left|r-r_{+}\right|^{-2}$.

Keywords: Colombeau nonlinear generalized functions; distributional riemannian geometry; distributional schwarzshild geometry; schwarzchild singularity; schwarzschild horizon; smooth regularization; nonsmooth regularization; quantum fields on curved spacetime; vacuum fluctuations; vacuum dominance.

2010 Mathematics Subject Classification: 53C25, 83C05, 57N16.

\section{Introduction}

\subsection{The breakdown of canonical formalism of riemann geometry for the singular solutions of the einstein field equations}

Einstein field equations were originally derived by Einstein in 1915 in respect with canonical formalism of Riemann geometry, i.e. by using the classical sufficiently smooth metric tensor, smooth Riemann curvature tensor, smooth Ricci tensor, smooth scalar curvature, etc. However singular solutions of the Einstein field equations with singular metric tensor and singular Riemann curvature tensor have soon been found. These singular solutions are formally accepted beyond rigorous canonical formalism of Riemannian geometry.

Remark 1.1 Note that if some components of the Riemann curvature tensor $R_{k l m}^{i}(\hat{x})$ become infinite at point $\hat{x}^{0}$ one obtains the breakdown of canonical formalism of Riemann geometry in a sufficiently small neighborhood $\Omega$ of the point $\hat{x}^{0} \in \Omega$, i.e. in such neighborhood $\Omega$ Riemann curvature tensor $R_{k l m}^{i}(\hat{x})$ will be changed by formula (1.7) see remark 1.2 .

Remark 1.2 Let $\Gamma$ be infinitesimal closed contour and let $\Sigma_{\Gamma}$ be the corresponding surface spanning by $\Gamma$,see Pic. 1 . We assume now that: (i) Christoffel symbol $\Gamma_{k l}^{i}(\hat{x})$ becomes infinite at singular point $\hat{x}^{0}$ by formulae

$$
\left\{\begin{array}{c}
\Gamma_{k l}^{i}(\hat{x}) \asymp \Xi_{k l}(\hat{x})\left(x_{i}-x_{i}^{0}\right)^{-\delta}, \delta \geq 1 \\
\Xi_{k l}(\hat{x}) \in C^{\infty}\left(\Sigma_{\Gamma}\right)
\end{array}\right.
$$

and (ii) $\hat{x}^{0} \in \Sigma_{\Gamma}$. Let us derive now to similarly canonical calculation [8], [9] the general formula for the regularized change $\widehat{\text { DeltaA}_{k}}$ in a vector $A_{i}(\hat{x})$ after parallel displacement around infinitesimal closed contour $\Gamma$.This regularized change $\widetilde{\Delta A_{k}}$ can clearly be written in the form

$$
\widetilde{\Delta A_{k}}=\int_{\Gamma} \Phi\left(\hat{x}-\hat{x}^{0}\right) \delta A_{k}
$$

where $\Phi\left(\hat{x}-\hat{x}^{0}\right)={ }_{i=0}^{4}\left(x_{i}-x_{i}^{0}\right)^{2 \delta}, \delta \geq 1$ and where the integral is taken over the given contour $\Gamma$. Substituting in place of $\delta A_{k}$ the canonical expression $\delta A_{k}=\Gamma_{k l}^{i}(\hat{x}) A_{k} d x^{l}$ (see [9], Eq. (85.5)) we obtain

$$
\begin{aligned}
& \widetilde{\Delta A_{k}}=\int_{\Gamma} \Phi\left(\hat{x}-\hat{x}^{0}\right) \delta A_{k}=\int_{\Gamma} \Phi\left(\hat{x}-\hat{x}^{0}\right) \Gamma_{k l}^{i}(\hat{x}) A_{k} d x^{l}, \\
& \text { where } \frac{\partial A_{i}}{\partial x^{l}}=\Gamma_{k l}^{i}(\hat{x}) A_{k} .
\end{aligned}
$$




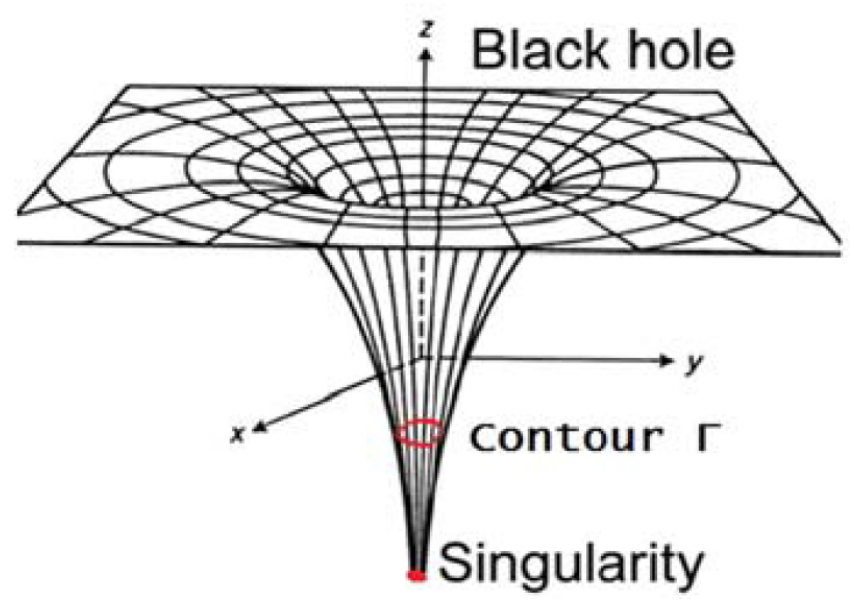

Pic. 1. Infinitesimal closed contour $\Gamma$ and corresponding singular surface $\Sigma_{\Gamma} \ni$ $\hat{x}^{0}$ spanning by $\Gamma$.

Due to the degeneracy of the metric (1.12) at point $\mathrm{r}=0$, the Levi-Civita' connection $\Gamma_{k j}^{+l}(\{\})=$

$$
=\frac{1}{2}\left[g^{l m}(\{\})\right]\left[\left(g_{m k, j}(\{\})+g_{m j, k}(\{\})-g_{k j, m}(\{\})\right]\right.
$$

is not available on $\mathrm{R}_{+}^{3} \cup\{0\}$ in canonical sense but only in an distributional sense.

Now applying Stokes' theorem (see [9], Eq. (6.19)) to the integral (1.3) and considering that the area enclosed by the contour has the infinitesimal value $\Delta f^{l m}$, we get

$$
\begin{aligned}
&= \frac{1}{2} \int_{\Sigma} \Gamma\left[\frac{\partial\left(\Gamma_{k m}^{i}(\hat{x}) A_{i} \Phi\left(\hat{x}-\hat{x}^{0}\right)\right)}{\partial x^{l}}-\frac{\partial\left(\Gamma_{k l}^{i}(\hat{x}) A_{i} \Phi\left(\hat{x}-\hat{x}^{0}\right)\right)}{\partial x^{m}}\right] d f^{l m} \approx \\
& \approx {\left[\frac{\partial\left(\Gamma_{k m}^{i}(\hat{x}) A_{i} \Phi\left(\hat{x}-\hat{x}^{0}\right)\right)}{\partial x^{l}}-\frac{\left.\partial\left(\Gamma_{k l}^{i}(\hat{x}) A_{i} \Phi\left(\hat{x}-\hat{x}^{0}\right)\right)\right]}{\partial x^{m}}\right] \frac{\Delta f^{l m}}{2}=} \\
& {\left[\Phi\left(\hat{x}-\hat{x}^{0}\right) \frac{\partial\left(\Gamma_{k m}^{i}\left(\hat{x}-\hat{x}^{0}\right) A_{i}\right)}{\partial x^{l}}+\left(\Gamma_{k m}^{i}(\hat{x}) A_{i}\right) \frac{\partial \Phi\left(\hat{x}-\hat{x}^{0}\right)}{\partial x^{l}}-\right.} \\
&\left.-\Phi\left(\hat{x}-\hat{x}^{0}\right) \frac{\partial\left(\Gamma_{k l}^{i}(\hat{x}) A_{i}\right)}{\partial x^{m}}-\left(\Gamma_{k l}^{i}(\hat{x}) A_{i}\right) \frac{\left.\partial \Phi\left(\hat{x}-\hat{x}^{0}\right)\right]}{\partial x^{m}}\right] \frac{\Delta f^{l m}}{2}= \\
& {\left[\Phi\left(\hat{x}-\hat{x}^{0}\right) \frac{\partial\left(\Gamma_{k m}^{i}(\hat{x}) A_{i}\right)}{\partial x^{l}}-\Phi\left(\hat{x}-\hat{x}^{0}\right) \frac{\partial\left(\Gamma_{k l}^{i}(\hat{x}) A_{i}\right)}{\partial x^{m}}-\right.} \\
&\left.A_{i}(\hat{x}) \Phi\left(\hat{x}-\hat{x}^{0}\right) \frac{2 \delta \Gamma_{k m}^{i}(\hat{x})}{x_{l}-x_{l}^{0}}-A_{i}(\hat{x}) \Phi\left(\hat{x}-\hat{x}^{0}\right) \frac{2 \delta \Gamma_{k l}^{i}(\hat{x})}{x_{m}-x_{m}^{0}}\right] \frac{\Delta f^{l m}}{2} .
\end{aligned}
$$

Substituting the values of the derivatives (1.4) into Eq. (1.5) we get finally:

$$
\widetilde{\Delta A_{k}}=\widetilde{R}_{k l m}^{i} \frac{A_{i}(\hat{x}) \Phi\left(\hat{x}-\hat{x}^{0}\right) \Delta f^{l m}}{2},
$$

where $\widetilde{R}_{k l m}^{i}$, is a tensor of the fourth rank

$$
\widetilde{R}_{k l m}^{i}=R_{k l m}^{i}+2 \delta\left[\frac{\Gamma_{k m}^{i}(\hat{x})}{x_{l}-x_{l}^{0}}-\frac{\Gamma_{k l}^{i}(\hat{x})}{x_{m}-x_{m}^{0}}\right] \text {. }
$$




\section{Black Hole Regions}

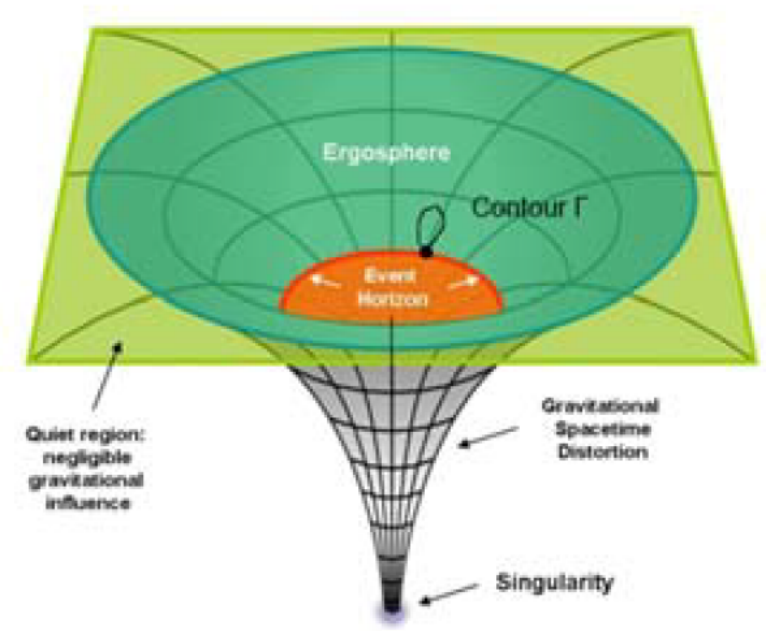

Pic. 2. Infinitesimal closed contour $\Gamma$ with a singularity at point $\hat{x}^{0}$ on Horizon and corresponding singular surface $\Sigma_{\Gamma} \ni \hat{x}^{0}$ spanning by $\Gamma$.

Due to the deheneracy of the metric (1.12) at $\mathrm{r}=2 \mathrm{~m}$, the Levi-Civita' connection $\Gamma_{k j}^{+l}(\{\})=$ $=\frac{1}{2}\left[g^{l m}(\{\})\right]\left[\left(g_{m k, j}(\{\})+g_{m j, k}(\{\})-g_{k j, m}(\{\})\right]\right.$

is not available on horizon in canonical sense but only in an distributional sense.

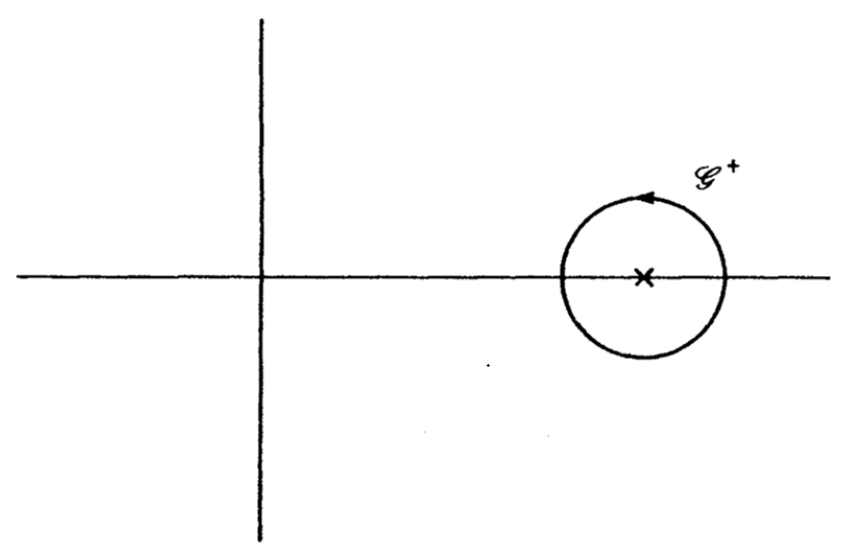

Pic. 3. The contour in the complex $\mathrm{k}^{0}$ plane $\mathbb{C}$ to be used in the evaluation of the integral giving $\mathscr{L}^{+}$. The cross indicates the pole at $\mathrm{k}^{0}=\left(|\mathbf{k}|^{2}+m^{2}\right)^{1 / 2}$. 
Here $R_{k l m}^{i}$ is the classical Riemann curvature tensor. That $\widetilde{R}_{k l m}^{i}$ is a tensor is clear from the fact that in (1.6) the left side is a vector - the difference $\widetilde{\Delta A_{k}}$ between the values of vectors at one and the same point. Note that an similar results were obtained by many authors [3], [4], [5], [6], [7], [10], [11], [12], [13], [14], [15], [16], [17] by using Colombeau nonlinear generalized functions [1]-[2], .

Definition 1.1. The tensor $\widetilde{R}_{k l m}^{i}$ is called the generalized curvature tensor or the generalized Riemann tensor.

Definition 1.2. The generalized Ricci curvature tensor $\widetilde{R}_{k m}$ is defined as

$$
\widetilde{R}_{k m}=\widetilde{R}_{k i m}^{i}
$$

Definition 1.3. The generalized Ricci scalar $\widetilde{R}$ is defined as

$$
\widetilde{R}=g^{k m} \widetilde{R}_{k m}
$$

Definition 1.4. The generalized Einstein tensor $\widetilde{G}_{k m}$ is defined as

$$
\widetilde{G}_{k m}=\widetilde{R}_{k m}-\frac{1}{2} g_{k m} \widetilde{R}
$$

Remark 1.3 (I) Note that the Schwarzschild spacetime is well defined only for $r>2 m$. The boundary of the mainfold $\{r>2 m\}$ in $\mathbb{R}^{3} \times \mathbb{R}$ is the submainfold $\{r=2 m\}$ of $\mathbb{R}^{3} \times \mathbb{R}$, diffeomorfic to a product $S^{2} \times \mathbb{R}$.

This submainfold is colled the event horizon, or simply the horizon [18], [19].

(II) The Schwarzschild metric (1.12) in canonical coordinates $\left(x^{0}, r, \theta\right.$, $\left.\phi\right)$, with $m>0$, ceases to be a smooth Lorentzian metric for $r=2 m$, because for such a value of $r$ the coefficient $g_{00}$ becomes zero while $g_{11}$ becomes infinite. For $0<r<2 m$ the metric (1.12) is again a smooth Lorentzian metric but tis a space coordinate while $r$ is a time coordinate. Hence the metric cannot be said to be either spherically symmetric or static for $r<2 m$. [18] (III) From consideration above obviously follows that on Schwarzschild spacetime

$$
\begin{gathered}
\text { Sh }=\left(S^{2} \times\{r>2 m\} \cup\{0<r<2 m\}\right) \times \mathbb{R} \text { the Levi-Civita connection } \\
\left\{\begin{array}{c}
\Gamma_{k j}^{+l}(\{\})==\frac{1}{2}\left[g^{l m}(\{\})\right]\left[\left(g_{m k, j}(\{\})+g_{m j, k}(\{\})-g_{k j, m}(\{\})\right]\right.
\end{array}\right.
\end{gathered}
$$

is not available in classical sence and that is well-known many years from mathematical literature, see for example [20] section 6 and Remark 1.1 - Remark 1.2 above.

(IV) Note that [19]: (i) The determinant $\operatorname{det}\left(g_{l m}(\{\})\right)=-r^{4} \sin ^{2} \theta$ of the metric (1.12) is reqular on horizon, i. e., smooth and non-vanishing for $r=2 m$. In addition:

(ii) The curvature scalar $\mathbf{R}(\{\})=g^{\mu \nu} \mathbf{R}_{\mu \nu}(\{\})$ is zero for $r=2 m$.

(iii) The none of higher-order scalars such as $\mathbf{R}^{\mu \nu}(\{\}) \mathbf{R}_{\mu \nu}(\{\})$, etc. blows up. For example the quadratic scalar $\mathbf{R}^{\rho \sigma \mu \nu}(\{\}) \mathbf{R}_{\rho \sigma \mu \nu}(\{\})=48 \mathrm{~m}^{2} / r^{6}$ is reqular on horizon, i. e., smooth and nonvanishing for $r=2 m$.

(V) Note that: (i) In physical literature (see for example [9], [18] and [21]. It was wrongly assumed that properties (i)-(iii) are enough to convince us that $r=2 m$ represent an non honest physical singularity but only coordinate singularity.

(VI) Such assumption based only on formal extensions $\widehat{\mathbf{R}}(\{\}), \widehat{\mathbf{R}}^{\mu \nu}(\{\}) \widehat{\mathbf{R}}_{\mu \nu}(\{\}), \ldots$, $\widehat{\mathbf{R}}^{\rho \sigma \mu \nu}(\{\}) \widehat{\mathbf{R}}_{\rho \sigma \mu \nu}(\{\})$ of the curvature scalar $\mathbf{R}(\{\})$ and higher-order scalars such as 


$$
\begin{gathered}
\mathbf{R}^{\mu \nu}(\{\}) \mathbf{R}_{\mu \nu}(\{\}), \ldots, \mathbf{R}^{\rho \sigma \mu \nu}(\{\}) \mathbf{R}_{\rho \sigma \mu \nu}(\{\}) \text { on horizon } r=2 m \text { and on origin } r=0 \text { by formulae } \\
\left.\widehat{\mathbf{R}}(r)\right|_{r=2 m}=\lim _{r \rightarrow 2 m} \mathbf{R}(r)=0,\left.\widehat{\mathbf{R}}(r)\right|_{r=0}=\lim _{r \rightarrow 0} \mathbf{R}(r)=0 \\
\left.\widehat{\mathbf{R}}^{\rho \sigma \mu \nu}(r) \widehat{\mathbf{R}}_{\rho \sigma \mu \nu}(r)\right|_{r=2 m}=\lim _{r \rightarrow 2 m}\left(\mathbf{R}^{\rho \sigma \mu \nu}(r) \mathbf{R}_{\rho \sigma \mu \nu}(r)\right)=\lim _{r \rightarrow 2 m} \frac{48 m^{2}}{r^{6}}=\left.\frac{48 m^{2}}{r^{6}}\right|_{r=2 m}, \\
\left.\widehat{\mathbf{R}}^{\rho \sigma \mu \nu}(r) \widehat{\mathbf{R}}_{\rho \sigma \mu \nu}(r)\right|_{r=0}=\lim _{r \rightarrow 0}\left(\mathbf{R}^{\rho \sigma \mu \nu}(r) \mathbf{R}_{\rho \sigma \mu \nu}(r)\right)=\lim _{r \rightarrow 0} \frac{48 m^{2}}{r^{6}}=\infty .
\end{gathered}
$$

However in the limit $\mathrm{r} \rightarrow 2 m$ the Levi-Civita connection $\Gamma_{k j}^{+l}(\{\})$ becomes infinite:

$$
\begin{aligned}
& \left.\Gamma_{00}^{1}(r)\right|_{r=2 m}=\lim _{r \rightarrow 2 m} \frac{m(r-2 m)}{r^{3}}=0,\left.\Gamma_{11}^{1}(r)\right|_{r=2 m}=\lim _{r \rightarrow 2 m} \frac{-m}{r(r-2 m)}=\infty, \\
& \left.\Gamma_{01}^{0}(r)\right|_{r=2 m}=\lim _{r \rightarrow 2 m} \frac{m}{r(r-2 m)}=\infty, \\
& \left.\Gamma_{12}^{2}(r)\right|_{r=2 m}=\lim _{r \rightarrow 2 m} \frac{1}{r}=2^{-1} m^{-1},\left.\Gamma_{22}^{1}\right|_{r=2 m}=-\lim _{r \rightarrow 2 m}(r-2 m)=0, \\
& \left.\Gamma_{13}^{3}\right|_{r=2 m}=\lim _{r \rightarrow 2 m} \frac{1}{r}=2^{-1} m^{-1},\left.\Gamma_{33}^{1}\right|_{r=2 m}=-\lim _{r \rightarrow 2 m}(r-2 m) \sin ^{2} \theta=0, \\
& \left.\Gamma_{00}^{1}(r)\right|_{r=0}=\lim _{r \rightarrow 0} \frac{m(r-2 m)}{r^{3}}=\left.\infty \Gamma_{11}^{1}(r)\right|_{r=0}=\lim _{r \rightarrow 0} \frac{-m}{r(r-2 m)}=\infty, \\
& \Gamma_{33}^{2}=-\sin \theta \cos \theta, \Gamma_{23}^{3}=\frac{\cos \theta}{\sin \theta} .
\end{aligned}
$$

Thus obviously by consideration above (see Remark 1.1 - Remark 1.2) this extension given by Eq. (1.15) has no any sense in respect of the canonical Riemannian geometry.

(VII) From consideration above (see Remark 1.1 - Remark 1.2) obviously follows that the scalars such as $\widehat{\mathbf{R}}(\{\}), \widehat{\mathbf{R}}^{\mu \nu}(\{\}) \widetilde{\mathbf{R}}_{\mu \nu}(\{\}), \ldots, \widehat{\mathbf{R}}^{\rho \sigma \mu \nu}(\{\}) \widehat{\mathbf{R}}_{\rho \sigma \mu \nu}(\{\})$ have no any sense in respect of the canonical Levi-Civita connection (1.11) and therefore cannot be said to be either honest physical singularity or only coordinate singularity in respect of the canonical Riemannian geometry.

Remark 1.4 Note that in physical literature the spacetime singularity usually is defined as location where the quantities that are used to measure the gravitational field become infinite in a way that does not depend on the coordinate system. These quantities are the classical scalar invariant curvatures of singular spacetime, which includes a measure of the density of matter.

Remark 1.5 In general relativity, many investigations have been derived with regard to singular exact vacuum solutions of the Einstein equation and the singilarity structure of space-time. Such solutions have been formally derived under condition $\mathbf{T}_{\mu}^{\nu}(x)=0$, where $\mathbf{T}_{\mu}^{\nu}(x)$ represent the energymomentum densities of the gravity source. This for example is the case for the well-known Schwarzschild solution, which is given by, in the Schwarzschild coordinates $\left(x^{0}, r, \theta, \phi\right)$,

$$
d s^{2}=-h(r)\left(d x^{0}\right)^{2}+h^{-1}(r)(d r)^{2}+r^{2}\left[(d \theta)^{2}+\sin ^{2} \theta(d \phi)^{2}\right], h(r)=1-\frac{r_{s}}{r},
$$

where $r_{s}$ is the Schwarzschild radius $r_{s}=2 G M / c^{2}$ with $G, M$ and $c$ being the Newton gravitational constant, mass of the source, and the light velocity in vacuum Minkowsky space-time, respectively. The metric (1.12) describes the gravitational field produced by a point-like particle located at $r=0$.

textbf Remark 1.6 Note that when we say, on the basis of the canonical expression of the curvature square

$$
\mathbf{R}^{\rho \sigma \mu \nu}(r) \mathbf{R}_{\rho \sigma \mu \nu}(r)=\frac{12 r_{s}^{2}}{r^{6}}
$$

fomally obtained from the metric (1.12), that $r=0$ is a singularity of the Schwarzschild space-time, 
the source is considered to be point-like and this metric is regarded as meaningful everywhere in space-time.

Remark 1.7 From the metric (1.12), the calculation of the canonical Einstein tensor proceeds in a straightforward manner gives for $r \neq 0 G_{t}^{t}(r)=G_{r}^{r}(r)=-\frac{h^{\prime}(r)}{\hat{r}}-\frac{1+h(r)}{\hat{r}^{2}} \equiv 0, G_{\theta}^{\theta}(r)=$ $G_{\varphi}^{\varphi}(r)=-\frac{h^{\prime \prime}(r)}{2}-\frac{h(r)}{\hat{r}^{2}} \equiv 0,(1.14)$ where $h(r)=-1+r_{s} / r$. Using Eq. (1.14) one formally obtains a boundary conditions

$$
\left\{\begin{aligned}
G_{t}^{t}(0) \triangleq \lim _{r \rightarrow 0} G_{t}^{t}(r)=0, G_{r}^{r}(0) \triangleq \lim _{r \rightarrow 0} G_{r}^{r}(r)=0 \\
G_{\theta}^{\theta}(0) \triangleq \lim _{r \rightarrow 0} G_{\theta}^{\theta}(r)=0, G_{\varphi}^{\varphi}(0) \triangleq \lim _{r \rightarrow 0} G_{\varphi}^{\varphi}(r)=0 .
\end{aligned}\right.
$$

However as pointed out above the canonical expression of the Einstein tensor in a sufficiently small neighborhood $\Omega$ of the point $r=0$ and must be replaced by the generalized Einstein tensor $\widetilde{G}_{k m}(1.10)$. By simple calculation easy to see that

$$
\left\{\begin{array}{l}
\widetilde{G}_{t}^{t}(0) \triangleq \lim _{r \rightarrow 0} \widetilde{G}_{t}^{t}(r)=-\infty, \widetilde{G}_{r}^{r}(0) \triangleq \lim _{r \rightarrow 0} \widetilde{G}_{r}^{r}(r)=-\infty, \\
\widetilde{G}_{\theta}^{\theta}(0) \triangleq \lim _{r \rightarrow 0} \widetilde{G}_{\theta}^{\theta}(r)=-\infty, \widetilde{G}_{\varphi}^{\varphi}(0) \triangleq \lim _{r \rightarrow 0} \widetilde{G}_{\varphi}^{\varphi}(r)=-\infty .
\end{array}\right.
$$

and therefore the boundary conditions (1.15) are completely wrong. But on the other hand as pointed out by many authors [3], [4], [5], [6], [7], [10], [11], [12], [13], [14], [15], [16], [17] that the canonical representation of the Einstein tensor, valid only in a weak (distributional) sense:

$$
G_{b}^{a}(\widehat{x})=-8 \pi m \delta_{0}^{a} \delta_{b}^{0} \delta^{3}(\widehat{x})
$$

and therefore we obtain again $G_{b}^{a}(0)=-\infty \times\left(\delta_{0}^{a} \delta_{b}^{0}\right)$.Thus canonical definition of the Einstein tensor is breakdown in rigorous mathematical sense for the Schwarzschild solution at origin $r=0$.

\subsection{The distributional schwarzschild geometry}

General relativity as a physical theory is governed by particular physical equations; the focus of interest is the breakdown of physics which need not coincide with the breakdown of geometry. It has been suggested to describe singularity at the origin as internal point of the Schwarzschild spacetime, where the Einstein field equations are satisfied in a weak (distributional) sense [3], [4], [5], [6], [7], [10], [11], [12], [13], [14], [15], [16], [17], [20], [22], [23], [24], [25].

\subsubsection{The smooth regularization of the singularity at the origin}

The two singular functions we will work with throughout this paper (namely the singular components of the Schwarzschild metric) are $\frac{1}{r}$ and $\frac{1}{r-r_{s}}, r_{s} \geq 0$. Since $\frac{1}{r} \in L_{l o c}^{1}\left(\mathbb{R}^{3}\right)$,it obviously gives the regular distribution $\frac{1}{r} \in D^{\prime}\left(\mathbb{R}^{3}\right)$. By convolution with a mollifier $3 c 1(x)$ adapted to the symmetry of the spacetime, (i.e. chosen radially symmetric) we embed it into the Colombeau algebra $\mathcal{G}\left(\mathbb{R}^{3}\right)$ [20]:

$$
\frac{1}{r} \stackrel{\iota}{\rightarrow} \iota\left(\frac{1}{r}\right) \triangleq\left(\frac{1}{r}\right) * \rho_{\varepsilon} \triangleq\left(\frac{1}{r}\right)_{\varepsilon}, \rho_{\varepsilon}=\frac{1}{\varepsilon^{3}} 3 c 1\left(\frac{r}{\varepsilon}\right), \varepsilon \in(0,1] .
$$

Inserting (1.18) into (1.12) we obtain a generalized Colombeau object modeling the singular Schwarzschild spacetime:

$$
\left\{\begin{array}{c}
\left(d s_{\varepsilon}^{2}\right)_{\varepsilon}=\left(h_{\varepsilon}(r)(d t)^{2}\right)_{\varepsilon}-\left(h_{\varepsilon}^{-1}(r)(d r)^{2}\right)_{\varepsilon}+r^{2}\left[(d \theta)^{2}+\sin ^{2} \theta(d \phi)^{2}\right], \\
h_{\varepsilon}(r)=-1+r_{s}\left(\frac{1}{r}\right)_{\varepsilon}, \varepsilon \in(0,1] .
\end{array}\right.
$$




\section{Remark 1.8}

Note that under regularization (1.18) for any $\operatorname{cin}(0,1]$ themetric

$$
d s_{\varepsilon}^{2}=h_{\varepsilon}(r)(d t)^{2}-h_{\varepsilon}^{-1}(r)(d r)^{2}+r^{2}\left[(d \theta)^{2}+\sin ^{2} \theta(d \phi)^{2}\right]
$$

obviously is a classical Riemannian object and there is no exist the breakdown of canonical formalism of Riemannian geometry for these metrics, even at origin $r=0$. It has been suggested by many authors to describe singularity at the origin as an internal point, where the Einstein field equations are satisfied in a distributional sense [3], [4], [5], [6], [7], [10], [11], [12], [13], [14], [15], [16], [17], [20], [22], [23], [24], [25]. From the Colombeau metric (1.19) one obtains in a distributional sense [20]:

$$
\begin{aligned}
& \left(R_{2}^{2}(r, \varepsilon)\right)_{\varepsilon}=\left(R_{3}^{3}\right)_{\varepsilon}=\left(\frac{h_{\varepsilon}^{\prime}(r)}{r}+\frac{1+h_{\varepsilon}(r)}{r^{2}}\right)_{\varepsilon}=8 \pi m \frac{\delta(r)}{r^{2}} \\
& \left(R_{0}^{0}(r, \varepsilon)\right)_{\varepsilon}=\left(R_{1}^{1}\right)_{\varepsilon}=\frac{1}{2}\left(\frac{h_{\varepsilon}^{\prime \prime}(r)}{2}+\frac{h_{\varepsilon}^{\prime}(r)}{r}\right)_{\varepsilon}=-4 \pi m \delta \frac{\delta(r)}{r^{2}} .
\end{aligned}
$$

Hence, the distributional Ricci tensor and the distributional curvature scalar $\left(R_{\varepsilon}(r)\right)_{\varepsilon}$ are of $\delta$-type, i.e. $\left(R_{\varepsilon}(r)\right)_{\varepsilon}=\pi m \frac{\delta(r)}{r^{2}}$.

\section{Remark 1.9}

Note that the formulae (1.20) should be contrasted with what is the expected result $G_{b}^{a}(x)=$ $-8 \pi m \delta_{0}^{a} \delta_{b}^{0} \delta^{3}(x)$ given by Eq.(1.17). However equations (1.20) are obviously given in spherical coordinates and therefore strictly speaking this is not correct, because the basis fields $\left\{\frac{\partial}{\partial r}, \frac{\partial}{\partial \varphi}, \frac{\partial}{\partial \theta}\right\}$ are not globally defined. Representing distributions concentrated at the origin require a basis regular at the origin. Transforming the formulae for $\left(R_{i j}(\varepsilon)\right)_{3 b 5}$ into Cartesian coordinates associated with the spherical ones, i.e., $\{r, \theta, \varphi\} \leftrightarrow\left\{x^{i}\right\}$, we obtain, e.g., for the Einstein tensor the expected result $G_{b}^{a}(x)=-8 \pi m \delta_{0}^{a} \delta_{b}^{0} \delta^{3}(x)$ given by Eq.(1.17) see [20].

\subsubsection{The nonsmooth regularization of the singularity at the origin}

The nonsmooth regularization of the Schwarzschild singularity at the origin $r=0$ is considered by N. R. Pantoja and H. H. Rago in [14 ]paper Pantoja nonsmooth regularization of the Schwarzschild singularity is

$$
\left(h_{\varepsilon}(r)\right)_{\varepsilon}=-1+\left(\frac{r_{s}}{r} \Theta(r-\varepsilon)\right)_{\varepsilon}, \varepsilon \in(0,1], r<r_{s} .
$$

Here $\Theta(u)$ is the Heaviside function and the limit $\varepsilon \rightarrow 0$ is understood in a distributional sense. Equation (1.19) with $h_{\varepsilon}$ as given in (1.21) can be considered as an regularized version of the Schwarzschild line element in cirvature coordinates From equation (1.21), the calculation of the distributional Einstein tensor proceeds in a straightforward manner. By simple calculation it gives [14]:

$$
\left\{\begin{aligned}
&\left(G_{t}^{t}(r, \varepsilon)\right)_{\varepsilon}=\left(G_{r}^{r}(r, \varepsilon)\right)_{\varepsilon}=-\left(\frac{h_{\varepsilon}^{\prime}(r)}{r}\right)_{\varepsilon}-\left(\frac{1+h_{\varepsilon}(r)}{r^{2}}\right)_{\varepsilon}= \\
&=-r_{s}\left(\frac{\delta(r-\varepsilon)}{r^{2}}\right)_{\varepsilon}=-r_{s} \frac{\delta(r)}{r^{2}}
\end{aligned}\right.
$$

and 


$$
\left\{\begin{array}{c}
\left(G_{\theta}^{\theta}(r, \varepsilon)\right)_{\varepsilon}=\left(G_{\varphi}^{\varphi}(r, \varepsilon)\right)_{\varepsilon}=-\left(\frac{h_{\varepsilon}^{\prime \prime}(r)}{2}\right)_{\varepsilon}-\left(\frac{h_{\varepsilon}(r)}{r^{2}}\right)_{\varepsilon}= \\
r_{s}\left(\frac{\delta(r-\varepsilon)}{r^{2}}\right)_{\varepsilon}-r_{s}\left(\frac{\varepsilon}{r^{2}} \frac{d}{d r} \delta(r-\varepsilon)\right)_{\varepsilon}=-r_{s} \frac{\delta(r)}{r^{2}} .
\end{array}\right.
$$

which is exactly the result obtained in [11] using smoothed versions of the Heaviside function $\Theta(r-\varepsilon)$. Transforming now the formulae for $\left(G_{b}^{a}(r, \varepsilon)\right)_{\epsilon}$ into Cartesian coordinates associated with the spherical ones, i.e., $\{r, \theta, \phi\} \leftrightarrow\left\{x^{i}\right\}$, we obtain for the generalized Einstein tensor the expected result given by Eq. (1.17).

$$
G_{b}^{a}(x)=-8 \pi m \delta_{0}^{a} \delta_{b}^{0} \delta^{3}(x),
$$

see Remark 1.9.

\subsubsection{The smooth regularization via horizon}

The smooth regularization via Horizon is considered by J. M. Heinzle and R. Steinbauer in [20] paper. Note that $\frac{1}{r-r_{s}} \notin L_{l o c}^{1}\left(\mathbb{R}^{3}\right)$. An canonical regularization is the principal value $\mathbf{v p}\left(\frac{1}{r-r_{s}}\right) \in$ $D^{\prime}\left(\mathbb{R}^{3}\right)$ which can be embedded into $\mathcal{G}\left(\mathbb{R}^{3}\right)[20]$ :

$$
\frac{1}{r-r_{s}} \stackrel{\mathbf{v p}}{\rightarrow} \mathbf{v p}\left(\frac{1}{r-r_{s}}\right) \stackrel{\iota}{\rightarrow} \iota\left[\rho_{\varepsilon} * \mathbf{v p}\left(\frac{1}{r-r_{s}}\right)\right] \triangleq\left(\frac{1}{r-r_{s}}\right)_{\varepsilon} \in \mathcal{G}\left(\mathbb{R}^{3}\right) \text {. }
$$

Inserting now (1.25) into (1.12) we obtain a generalized Colombeau object modeling the singular Schwarzschild spacetime [20]:

$$
\left\{\begin{array}{c}
\left(d s_{\varepsilon}^{2}\right)_{\varepsilon}=\left(h(r)(d t)^{2}\right)_{\varepsilon}-\left(h_{\varepsilon}^{-1}(r)(d r)^{2}\right)_{\varepsilon}+r^{2}\left[(d \theta)^{2}+\sin ^{2} \theta(d \phi)^{2}\right], \\
h(r)=-1+\frac{r_{s}}{r}, h_{\varepsilon}^{-1}(r)=-1-r_{s}\left(\frac{1}{r-r_{s}}\right)_{\varepsilon}, \varepsilon \in(0,1] .
\end{array}\right.
$$

Remark 1.10 Note that obviously Colombeau object, (1.26) is degenerated at $r=r_{s}$, because $h(r)$ is zero at the horizon. However, this does not come as a surprise. Both $h(r)$ and $h^{-1}(r)$ are positive outside of the black hole and negative in the interior. As a consequence any smooth regularization of $h(r) \quad$ (or $h^{-1}$ ) must pass through zero somewhere and, additionally, this zero must converge to $r=r_{s}$ as the regularization parameter goes to zero.

Remark 1.11 Note that due to the degeneracy of Colombeau object (1.26), even the distributional Levi-Civitá connection obviously is not available by using the smooth regularization via horizon $[20]$.

\subsubsection{The nonsmooth regularization via horizon}

In this paper we leave the neighborhood of the singularity at the origin and turn to the singularity at the horizon. The question we are aiming at is the following: using distributional geometry (thus without leaving Schwarzschild coordinates), is it possible to show that the horizon singularity of the Schwarzschild metric is not merely a coordimate singularity. In order to investigate this issue we calculate the distributional curvature at the horizon in Schwarzschild coordinates.

The main focus of this work is a (nonlinear) superdistributional description of the Schwarzschild spacetime. Although the nature of the Schwarzschild singularity is much "worse" than the quasiregular conical singularity, there are several distributional treatments in the literature [6], [7], [10], $[11],[12],[13],[14],[15],[16],[17],[20],[22],[23],[24],[25],[26],[27],[28],[29],[30]$, mailnly motivated by the following considerations: the physical interpretation of the Schwarzschild metric is clear as long as we consider it merely as an exterior (vacuum) solution of an extended (sufficiently 
large) massive spherically symmetric body. Together with the interior solution it describes the entire spacetime. The concept of point particles - well understood in the context of linear field theories - suggests a mathematical idealization of the underlying physics: one would like to view the Schwarzschild solution as defined on the entire spacetime and regard it as generated by a point mass located at the origin and acting as the gravitational source.

This of course amounts to the question of whether one can reasonably ascribe distributional curvature quantities to the Schwarzschild singularity at the horizon.

The emphasis of the present work lies on mathematical rigor. We derive the "physically expected" result for the distributional energy momentum tensor of the Schwarzschild geometry, i. e., $T_{0}^{0}=$ $8 \pi m \delta^{(3)}(\vec{x})$,in a conceptually satisfactory way. Additionally, we set up a unified language to comment on the respective merits of some of the approaches taken so far. In particular, we discuss questions of differentiable structure as well as smoothness and degeneracy problems of the regularized metrics, and present possible refinements and workarounds. These aims are accomplished using the framework of nonlinear supergeneralized functions (supergeneralized Colombeau algebras $\widetilde{\mathcal{G}}\left(\mathbb{R}^{3}, \Sigma\right)$ ). Examining the Schwarzschild metric (1.12) in a neighborhood of the horizon, we see that, whereas $h(r)$ is smooth, $h^{-1}(r)$ is not even $L_{l o c}^{1}$ (note that the origin is now always excluded from our considerations; the space we are working on is $\left.\mathbb{R}^{3} \backslash\{0\}\right)$. Thus, regularizing the Schwarzschild metric amounts to embedding $h^{-1}$ into $\widetilde{\mathcal{G}}\left(\mathbb{R}^{3}, \Sigma\right.$ ) (as done in (3.2)). Obviously, (3.1) is degenerate at $r=2 m$, because $h(r)$ is zero at the horizon. However, this does not come as a surprise. Both $h(r)$ and $h^{-1}(r)$ are positive outside of the black hole and negative in the interior. As a consequence any (smooth) regularization $h_{\epsilon}^{+}(r)\left(h_{\epsilon}^{-}(r)\right)$ [above (below) horizon] of $h(r)$ must pass through small enough vicinity

$$
\begin{aligned}
& O_{\epsilon}^{+}(2 m)=\left\{\vec{x} \in \mathbb{R}^{3} \mid\|\vec{x}\|>2 m,\|\vec{x}-2 m\| \leqslant \epsilon\right\} \\
& \quad\left(O_{\epsilon}^{-}(2 m)=\left\{\vec{x} \in \mathbb{R}^{3} \mid\|\vec{x}\|<2 m,\|\vec{x}-2 m\| \leqslant \epsilon\right\}\right)
\end{aligned}
$$

of zeroz set $O_{0}(2 m)=\left\{\vec{y} \in \mathbb{R}^{3} \mid\|\vec{y}\|=2 m\right\}$ somewhere and, additionally, this vicinity $O_{\epsilon}^{+}(2 m)$ $\left(O_{\epsilon}^{-}(2 m)\right)$ must converge to $O_{0}(2 m)$ as the regularization parameter $\epsilon$ goes to zero. Due to the degeneracy of (1.12), the Levi-Civita connection is not available. By appropriate nonsmooth regularization (see section 3) we obtain a Colombeau generalized object modeling the singular Schwarzschild metric above and below horizon, i. e.,

$$
\left\{\begin{array}{c}
\left(d s_{\epsilon}^{+2}\right)_{\epsilon}=\left(h_{\epsilon}^{+}(r) d t^{2}\right)_{\epsilon}-\left(\left[h_{\epsilon}^{+}(r)\right]^{-1} d r^{2}\right)_{\epsilon}+r^{2} d \Omega^{2}, \\
\left(d s_{\epsilon}^{-2}\right)_{\epsilon}=\left(h_{\epsilon}^{-}(r) d t^{2}\right)_{\epsilon}-\left(\left[h_{\epsilon}^{-}(r)\right]^{-1} d r^{2}\right)_{\epsilon}+r^{2} d \Omega^{2}, \\
\epsilon \in(0,1] .
\end{array}\right.
$$

Consider corresponding distributional connections

$$
\begin{aligned}
&\left(\Gamma_{k j}^{+l}(\epsilon)\right)_{\epsilon}=\left(\Gamma_{k j}^{+l}\left[h_{\epsilon}^{+}\right]\right)_{\epsilon} \in \widetilde{\mathcal{G}}\left(\mathbb{R}^{3}, \Sigma\right) \text { and }\left(\Gamma_{k j}^{-l}(\epsilon)\right)_{\epsilon}=\left(\Gamma_{k j}^{-l}\left[h_{\epsilon}^{+}\right]\right)_{\epsilon} \in \widetilde{\mathcal{G}}\left(\mathbb{R}^{3}, \Sigma\right): \\
&\left(\Gamma_{k j}^{+l}(\epsilon)\right)_{\epsilon}=\frac{1}{2}\left(\left(g_{\epsilon}^{+l m}\right)\left[\left(g_{\epsilon}^{+}\right)_{m k, j}+\left(g_{\epsilon}^{+}\right)_{m j, k}-\left(g_{\epsilon}^{+}\right)_{k j, m}\right]\right)_{\epsilon}, \\
&\left(\Gamma_{k j}^{-l}(\epsilon)\right)_{\epsilon}=\frac{1}{2}\left(\left(g_{\epsilon}^{-l m}\right)\left[\left(g_{\epsilon}^{-}\right)_{m k, j}+\left(g_{\epsilon}^{-}\right)_{m j, k}-\left(g_{\epsilon}^{-}\right)_{k j, m}\right]\right)_{\epsilon} .
\end{aligned}
$$

Obviously $\left(\Gamma_{k j}^{+l}\left[h_{\epsilon}^{+}\right]\right)_{\epsilon},\left(\Gamma_{k j}^{-l}\left[h_{\epsilon}^{+}\right]\right)_{\epsilon}$ coincides with the corresponding Levi-Civita connection on $\mathbb{R}^{3} \backslash\{(r=0) \cup(r=2 m)\}$, as $\left(h_{\epsilon}^{+}\right)_{\epsilon}=h_{0}^{+},\left(h_{\epsilon}^{-}\right)_{\epsilon}=h_{0}^{-}$, and $\left(g_{\epsilon}^{+l m}\right)_{\epsilon}=g_{0}^{+l m},\left(g_{\epsilon}^{-l m}\right)_{\epsilon}=g_{0}^{-l m}$ there. Clearly, connections $\Gamma_{k j}^{+l}(\epsilon), \Gamma_{k j}^{-l}(\epsilon), \epsilon \in(0,1]$ are in respect of the regularized metric $g_{\epsilon}^{ \pm}, \epsilon \in(0,1]$, i.e., $\left(g_{\epsilon}^{ \pm}\right)_{i j ; k}=0$. Proceeding in this manner, we obtain the nonstandard result 


$$
\left\{\begin{array}{c}
\left(\left[\mathbf{R}_{\epsilon}^{+}\right]_{1}^{1}\right)_{\epsilon}=\left(\left[\mathbf{R}_{\epsilon}^{+}\right]_{0}^{0}\right)_{\epsilon} \approx-m \tilde{\Phi}(2 m), \\
\left(\left[\mathbf{R}_{\epsilon}^{-}\right]_{1}^{1}\right)_{\epsilon}=\left(\left[\mathbf{R}_{\epsilon}^{-}\right]_{0}^{0}\right)_{\epsilon} \simeq m \tilde{\Phi}(2 m) .
\end{array}\right.
$$

Investigating the weak limit of the angular components of the generalized Ricci tensor using the abbreviation

$$
\tilde{\Phi}(r)={ }_{0}^{\pi} \sin \theta d \theta_{0}^{2 \pi} d \phi \Phi(x)
$$

and let $\Phi(x)$ be the function $\Phi(x) \in \mathcal{S}_{2 m}^{+}\left(\mathbb{R}^{3}\right)\left(\Phi(x) \in \mathcal{S}_{2 m}^{-}\left(\mathbb{R}^{3}\right)\right)$, where by $\mathcal{S}_{2 m}^{+}\left(\mathbb{R}^{3}\right)\left(\mathcal{S}_{2 m}^{-}\left(\mathbb{R}^{3}\right)\right.$ we denote the class of all functions $\Phi(x)$ with compact support such that

(i) $\operatorname{supp}(\Phi(x)) \subset\{x \mid\|x\| \geq 2 m\} \quad(\operatorname{supp}(\Phi(x)) \subset\{x \mid\|x\| \leq 2 m\})$ (ii) $\tilde{\Phi}(r) \in C^{\infty}(\mathbb{R})$. Then for any function $\Phi(x) \in \mathcal{S}_{2 m}^{ \pm}\left(\mathbb{R}^{3}\right)$ we get:

$$
\left\{\begin{array}{c}
w-\lim _{\epsilon \rightarrow 0}\left[\mathbf{R}_{\epsilon}^{+}\right]_{1}^{1}=w-\lim _{\epsilon \rightarrow 0}\left[\mathbf{R}_{\epsilon}^{+}\right]_{0}^{0}=m\langle\tilde{\delta} \mid \Phi\rangle=-m \tilde{\Phi}(2 m), \\
w-\lim _{\epsilon \rightarrow 0}\left[\mathbf{R}_{\epsilon}^{-}\right]_{1}^{1}=w-\lim _{\epsilon \rightarrow 0}\left[\mathbf{R}_{\epsilon}^{-}\right]_{0}^{0}=m\langle\tilde{\delta} \mid \Phi\rangle=m \tilde{\Phi}(2 m),
\end{array}\right.
$$

i. e., the Schwarzschild spacetime is weakly Ricii-nonflat (the origin was excluded from our considerations). Furthermore, the Tolman formula for the total energy of a static and asymptotically flat spacetime with $g$ the determinant of the four-dimensional metric and $d^{3} x$ the coordinate volume element gives

$$
E_{T}=\int\left(\mathbf{T}_{r}^{r}+\mathbf{T}_{\theta}^{\theta}+\mathbf{T}_{\phi}^{\phi}+\mathbf{T}_{t}^{t}\right) \sqrt{-g} d^{3} x=m
$$

as it should be.

The paper is organized in the following way: in section II we discuss the conceptual as well as the mathematical prerequisites. In particular we comment on geometrical matters (differentiable structure, coordinate invariance) and recall the basic facts of nonlinear superdistributional geometry in the context of algebras $\widetilde{\mathcal{G}}(M, \Sigma)$ of supergeneralized functions Moreover, we derive sensible nonsmooth regularizations of the singular functions to be used throughout the paper. Section III is devoted to these approaches to the problem. We present a new conceptually satisfactiry method to derive the main result. In this final section III we investigate the horizon and describe its distributional curvature. Using nonlinear superdistributional geometry and supergeneralized functions it semms possible to show that the horizon singularity is not only a coordinate singularity with out leaving Schwarzschild coordinates.

\subsubsection{Distributional eddington-finkelstein spacetime}

In physical literature for many years the belief that the Schwarzschild spacetime $\left(S^{2} \times\{r>2 m\}\right) \times$ $\mathbb{R}$ is extendible exists, in the sense that it can be immersed in a larger spaetime whose manifold is not covered by the canonical Schwarzschild coordinate with $r>2 m$. In physical literature [9], $[18],[19],[21]$ One considers the formal change of coordinates obtained by replacing the canonical Schwarzschild time by "retarded time" above horizon $\nu^{+}$given when $r>2 m$ by

$$
\nu^{+}=t+r+2 m \ln \left(\frac{r}{2 m}-1\right) \text {. }
$$

From (1.31) it follows for $r>2 m$

$$
d t=-\frac{d r}{1-\frac{2 m}{r}}+d v^{+}
$$

The Schwarzschild metric (1.12) above horizon $d s^{+2}$ (see section 3) in this coordinate obviously takes the form 


$$
d s^{+2}=-\left(1-\frac{2 m}{r}\right) d v^{+2}+2 d r d v^{-}+r^{2}\left[(d \theta)^{2}+\sin ^{2} \theta(d \phi)^{2}\right]
$$

When $r<2 m$ we replace (1.33) below horizon by

$$
\nu^{-}=t+r+2 m \ln \left(1-\frac{r}{2 m}\right)
$$

From (1.36) it follows for $r<2 m$

$$
d t=\frac{d r}{\frac{2 m}{r}-1}+d v^{-} .
$$

The Schwarzschild metric (1.12) below horizon $d s_{-}^{2}$ (see section 3) in this coordinate obviously takes the form

$$
d s^{-2}=\left(\frac{2 m}{r}-1\right) d v^{-2}-2 d r d v^{-}+r^{2}\left[(d \theta)^{2}+\sin ^{2} \theta(d \phi)^{2}\right] .
$$

Remark 1.12 (i) Note that the metric (1.33) is defined on the manifold $S^{2} \times(r>0) \times \mathbb{R}$ and obviously it is regular Lorentzian metric: its coefficients are smooth.

(ii) The term $2 d r d v$ ensures its non-degeneracy for $r=2 m$.

(iii) Due to the nondegeneracy of the metric (1.32) the Levi-Civita connection

$$
\left\{\Gamma_{k j}^{+l}(\{\})==\frac{1}{2}\left[g^{l m}(\{\})\right]\left[\left(g_{m k, j}(\{\})+g_{m j, k}(\{\})-g_{k j, m}(\{\})\right]\right.\right.
$$

is obviously now available and therefore nonsingular on horizon in contrast Schwarzschild metric (1.12) one obtains:

$$
\begin{gathered}
\Gamma_{\nu \nu}^{\nu}=\frac{r_{s}}{2 r^{2}}, \Gamma_{\nu \nu}^{r}=\frac{r_{s}\left(r-r_{s}\right)}{2 r^{3}}, \Gamma_{\nu r}^{r}=-\frac{r_{s}}{2 r^{2}}, \Gamma_{r \theta}^{\theta}=\frac{1}{r}, \\
\Gamma_{r \varphi}^{\varphi}=\frac{1}{r}, \Gamma_{\theta \theta}^{\nu}=-r, \Gamma_{\theta \theta}^{r}=-r\left(r-r_{s}\right), \Gamma_{\theta \varphi}^{\varphi}=\cot \theta, \\
\Gamma_{\varphi \varphi}^{\nu}=-r \sin ^{2} \theta, \Gamma_{\varphi \varphi}^{r}=-r\left(r-r_{s}\right) \sin ^{2} \theta, \Gamma_{\varphi \varphi}^{\theta}=-\sin \theta \cos \theta .
\end{gathered}
$$

(iv) In physical literature [9], [18], [19], [21] by using properties (i) - (iii) this spacetime wrongly convicted as an rigorous mathematical extension of the Schwarzschild spacetime.

Remark 1.13. Let us consider now the coordinates: (i) $v^{+}, r^{\prime}=r, \theta^{\prime}=\theta, \varphi^{\prime}=\varphi$ and (ii) $v^{-}, r^{\prime}=r, \theta^{\prime}=\theta, \varphi^{\prime}=\varphi$. Obviously both transformations given by Eq.(1.33) and Eq.(1.36 ) are singular because the both Jacobian of these transformations are singular at $r=2 m$ :

$$
\left(\begin{array}{ll}
\frac{\partial v^{+}}{\partial t} & \frac{\partial v^{+}}{\partial r^{\prime}} \\
\frac{\partial r^{\prime}}{\partial t} & \frac{\partial r^{\prime}}{\partial r}
\end{array}\right)=\left(\begin{array}{cc}
1 & \frac{r}{r-2 m} \\
0 & 1
\end{array}\right)
$$

and

$$
\left(\begin{array}{ll}
\frac{\partial v^{-}}{\partial t} & \frac{\partial v^{-}}{\partial r} \\
\frac{\partial r^{\prime}}{\partial t} & \frac{\partial r^{\prime}}{\partial r}
\end{array}\right)=\left(\begin{array}{cc}
1 & -\frac{r}{2 m-r} \\
0 & 1
\end{array}\right)
$$

Remark 1.14 Note first (i) such singular transformations are not allowed in conventional Lorentzian geometry and second (ii) both Eddington-Finkelstein metrics given by Eq.(1.35) and by Eq.(1.38) again are not well defined in any rigorous mathematical sense at $r=2 m$.

Remark 1.15 From consideration above it follows that Schwarzschild spacetime $\left(S^{2} \times\{r>2 m\}\right) \times$ $\mathbb{R}$ is not extendible, in the sense that it can be immersed in a larger spacetime whose manifold is 
not covered by the canonical Schwarzschild coordinate with $r>2 m$. Thus Eddington-Finkelstein spacetime cannot be considered as an extension of the Schwarzschild spacetime in any rigorous mathematical sense in respect conventional Lorentzian geometry. Such "extension" is the extension by abnormal definition and nothing more.

Remark 1.16. From consideration above it follows that it is necessary an regularization of the Eq.(1.34) and Eq.(1.37) on horizon. However obviously only nonsmooth regularization via horizon $r=2 m$ is possible. Under nonsmooth regularization( see section 3) Eq.(1.34) and Eq.(1.37) take the form

$$
\begin{gathered}
d t=-\frac{d r}{\frac{1}{r} \sqrt{(r-2 m)^{2}+\epsilon^{2}}}+d v_{\epsilon}^{+}, \\
\epsilon \in(0,1]
\end{gathered}
$$

and

$$
\begin{gathered}
d t=\frac{d r}{\frac{1}{r} \sqrt{(2 m-r)^{2}+\epsilon^{2}}}+d v_{\epsilon}^{-}, \\
\epsilon \in(0,1]
\end{gathered}
$$

correspondingly. Therefore Eq.(1.41) - Eq.(1.42) take the form

$$
\left(\begin{array}{ll}
\frac{\partial v_{\epsilon}^{+}}{\partial t} & \frac{\partial v_{\epsilon}^{+}}{\partial r} \\
\frac{\partial r^{\prime}}{\partial t} & \frac{\partial r^{\prime}}{\partial r}
\end{array}\right)=\left(\begin{array}{cc}
1 & \frac{r}{\sqrt{(r-2 m)^{2}+\epsilon^{2}}} \\
0 & 1
\end{array}\right)
$$

and

$$
\left(\begin{array}{ll}
\frac{\partial v_{\epsilon}^{-}}{\partial t} & \frac{\partial v_{\epsilon}^{-}}{\partial r} \\
\frac{\partial r^{\prime}}{\partial t} & \frac{\partial r^{\prime}}{\partial r}
\end{array}\right)=\left(\begin{array}{cc}
1 & -\frac{r}{\sqrt{(2 m-r)^{2}+\epsilon^{2}}} \\
0 & 1
\end{array}\right)
$$

From Eq. (1.43) - Eq. (1.44) one obtains generalized Eddington-Finkelstein transformations such as

$$
d t=-\frac{r d r}{\left(\sqrt{(r-2 m)^{2}+\epsilon^{2}}\right)_{\epsilon}}+\left(d v_{\epsilon}^{+}\right)_{\epsilon}
$$

and

$$
d t=\frac{r d r}{\left(\sqrt{(2 m-r)^{2}+\epsilon^{2}}\right)_{\epsilon}}+d v_{\epsilon}^{-},
$$

Therefore Eq.(1.45)-Eq.(1.46) take the form

$$
\left(\begin{array}{cc}
\left(\frac{\partial v_{\epsilon}^{+}}{\partial t}\right)_{\epsilon} & \left(\frac{\partial v_{\epsilon}^{+}}{\partial r}\right)_{\epsilon} \\
\frac{\partial r^{\prime}}{\partial t} & \frac{\partial r^{\prime}}{\partial r}
\end{array}\right)=\left(\begin{array}{cc}
1 & \frac{r}{\left(\sqrt{(r-2 m)^{2}+\epsilon^{2}}\right)_{\epsilon}} \\
0 & 1
\end{array}\right)
$$

and 


$$
\left(\begin{array}{cc}
\left(\frac{\partial v_{\epsilon}^{-}}{\partial t}\right)_{\epsilon} & \left(\begin{array}{c}
\left.\frac{\partial v_{\epsilon}^{-}}{\partial r}\right)_{\epsilon} \\
\frac{\partial r^{\prime}}{\partial t}
\end{array}\right. \\
\frac{\partial r^{\prime}}{\partial r}
\end{array}\right)=\left(\begin{array}{cc}
1 & -\frac{r}{\left(\sqrt{(2 m-r)^{2}+\epsilon^{2}}\right)_{\epsilon}}
\end{array}\right)
$$

At point $r=2 m$ one obtains

$$
\begin{aligned}
& \left.\qquad \begin{array}{cc}
\left(\frac{\partial v_{\epsilon}^{+}}{\partial t}\right)_{\epsilon} & \left(\frac{\partial v_{\epsilon}^{+}}{\partial r}\right)_{\epsilon} \\
\frac{\partial r^{\prime}}{\partial t} & \frac{\partial r^{\prime}}{\partial r}
\end{array}\right)\left.\right|_{r=2 m}=\left(\begin{array}{cc}
1 & r\left(\epsilon^{-1}\right)_{\epsilon} \\
0 & 1
\end{array}\right) \\
& \text { and } \\
& \qquad\left.\left(\begin{array}{cc}
\left(\frac{\partial v_{\epsilon}^{-}}{\partial t}\right)_{\epsilon} & \left(\begin{array}{c}
\frac{\partial v_{\epsilon}^{-}}{\partial r} \\
\frac{\partial r^{\prime}}{\partial t}
\end{array}\right. \\
\frac{\partial r^{\prime}}{\partial r}
\end{array}\right)\right|_{r=2 m}=\left(\begin{array}{cc}
1 & -r\left(\epsilon^{-1}\right)_{\epsilon} \\
0 & 1
\end{array}\right),
\end{aligned}
$$

where $\left(\epsilon^{-1}\right)_{\epsilon} \in \widetilde{\mathbb{R}}$. Thus generalized Eddington-Finkelstein transformations (1.47)-(1.48) are well defined in sense of Colombeau generalized functions. Therefore Colombeau generalized object modeling the classical Eddington-Finkelstein metric (1.35)-(1.36) above and below gorizon takes the form

$$
\begin{aligned}
& \left(d s_{\epsilon}^{+2}\right)_{\epsilon}=-\frac{1}{r} \sqrt{(r-2 m)^{2}+\epsilon^{2}} d v_{\epsilon}^{+2}+2 d r d v_{\epsilon}^{+}+r^{2}\left[(d \theta)^{2}+\sin ^{2} \theta(d \phi)^{2}\right] . \\
& \left(d s_{\epsilon}^{-2}\right)_{\epsilon}=-\frac{1}{r} \sqrt{(2 m-r)^{2}+\epsilon^{2}} d v_{\epsilon}^{-2}+2 d r d v_{\epsilon}^{-}+r^{2}\left[(d \theta)^{2}+\sin ^{2} \theta(d \phi)^{2}\right] .
\end{aligned}
$$

It easily to verify by using formula A.2 (see appendix) that the distributional curvature scalar $(\mathbf{R}(\epsilon))_{\epsilon}$ is singular at $r=2 m$ as in the case of the distributional Schwarzschild spacetime given by Eq.(1.28). However this is not surprising because the classical Eddington-Finkelstein spacetime and generalized Eddington-Finkelstein specetime given by Eq.(1.53) are essentially different geometrical objects.

\section{Generalized Colombeau Calculus}

\subsection{Notation and basic notions from standard Colombeau theory}

We use [1], [2] and [5] as standard references for the foundations and various applications of standard Colombeau theory. We briefly recall the basic Colombeau construction. Throughout the paper $\Omega$ will denote an open subset of $\mathbb{R}^{n}$. Standard Colombeau generalized functions on $\Omega$ are defined as equivalence classes $u=\left[\left(u_{\varepsilon}\right)_{\varepsilon}\right]$ of nets of smooth functions $u_{\varepsilon} \in C^{\infty}(\Omega)$ (regularizations) subjected to asymptotic norm conditions with respect to $\varepsilon \in(0,1]$ for their derivatives on compact sets.

The basic idea of classical Colombeau's theory of nonlinear generalized functions is regularization by sequences (nets) of smooth functions and the use of asymptotic estimates in terms of a regularization parameter $\varepsilon$. Let $\left(u_{\varepsilon}\right)_{\varepsilon \in(0,1]}$ with $\left(u_{\varepsilon}\right)_{\varepsilon} \in \mathcal{C}^{\infty}(M)$ for all $\varepsilon \in \mathbb{R}_{+}$, where $M$ is a separable, smooth orientable HausdorfF mainfold of dimensionn.

Definition 2.1. The classical Colombeau's algebra of generalized functions on $M$ is defined as the quotient:

$$
\mathcal{G}(M) \triangleq \mathcal{E}_{M}(M) / \mathcal{N}(M)
$$

ofthespace $\mathrm{E}_{M}(M)$ of sequences of moderate growth modulo the space $\mathcal{N}(M)$ of negligible sequences. More precisely the notions of moderateness resp. negligibility are defined by the following asymptotic 
estimates (where $\mathfrak{X}(M)$ denoting the space of smooth vector fields on $M$

$$
\begin{gathered}
\left\{\begin{array}{c}
\mathcal{E}_{M}(M) \triangleq\left\{\left(u_{\varepsilon}\right)_{\varepsilon} \mid \forall K(K \varsubsetneqq M) \forall k(k \in \mathbb{N}) \exists N(N \in \mathbb{N})\right. \\
\left.\forall \xi_{1}, \ldots, \xi_{k}\left(\xi_{1}, \ldots, \xi_{k} \in \mathfrak{X}(M)\right)\left[\sup _{p \in K}\left|L_{\xi_{1}} \ldots L_{\xi_{k}} u_{\varepsilon}(p)\right|=O\left(\varepsilon^{-N}\right) \text { as } \varepsilon \rightarrow 0\right]\right\}, \\
\mathcal{N}(M) \triangleq\left\{\left(u_{\varepsilon}\right)_{\varepsilon} \mid \forall K(K \varsubsetneqq M), \forall k\left(k \in \mathbb{N}_{0}\right) \forall q(q \in N)\right. \\
\left.\forall \xi_{1}, \ldots, \xi_{k}\left(\xi_{1}, \ldots, \xi_{k} \in \mathfrak{X}(M)\right)\left[\sup _{p \in K}\left|L_{\xi_{1}} \ldots L_{\xi_{k}} u_{\varepsilon}(p)\right|=O\left(\varepsilon^{q}\right) \text { as } \varepsilon \rightarrow 0\right]\right\} .
\end{array}\right.
\end{gathered}
$$

Remark 2.1 In the definition the Landau symbol $a_{\varepsilon}=O(\psi(\varepsilon))$ appears, having the following meaning: $\exists C(C>0) \exists \varepsilon_{0}\left(\varepsilon_{0} \in(0,1]\right) \forall \varepsilon\left(\varepsilon<\varepsilon_{0}\right)\left[a_{\varepsilon} \leq C \psi(\varepsilon)\right]$.

Definition 2.2. Elements of $\operatorname{calG}(M)$ are denoted by:

$$
u=\operatorname{cl}\left[\left(u_{\varepsilon}\right)_{\varepsilon}\right] \triangleq\left(u_{\varepsilon}\right)_{\varepsilon}+\mathcal{N}(M) .
$$

Remark 2.2. With componentwise operations $(\cdot, \pm) \mathcal{G}(M)$ is a fine sheaf of differential algebras with respect to the Lie derivative defined by $L_{\xi} u \triangleq \operatorname{cl}\left[\left(L_{\xi} u_{\varepsilon}\right)_{\varepsilon}\right]$.

The spaces of moderate resp. negligible sequences and hence the algebra itself may be characterized locally, i.e., $u \in \mathcal{G}(M)$ iff $u \circ \psi_{\alpha} \in \mathcal{G}\left(\psi_{\alpha}\left(V_{\alpha}\right)\right)$ for all charts $\left(V_{\alpha}, \psi_{\alpha}\right)$, where on the open set $\psi_{\alpha}\left(V_{\alpha}\right) \subset \mathbb{R}^{n}$ in the respective estimates Lie derivatives are replaced by partial derivatives.

Remark 2.3 Smooth functions $f \in \mathcal{C}^{\infty}(M)$ are embedded into $\mathcal{G}(M)$ simply by the "constant" embedding $\sigma$, i.e., $\sigma(f)=\mathbf{c l}\left[(f)_{\varepsilon}\right]$, hence $\mathcal{C}^{\infty}(M)$ is a faithful subalgebra of $\mathcal{G}(M)$.

\subsubsection{Point values of a generalized functions on $M$. generalized numbers}

Within the classical distribution theory, distributions cannot be characterized by their point values in any way similar to classical functions. On the other hand, there is a very natural and direct way of obtaining the point values of the elements of Colombeau's algebra: points are simply inserted into representatives. The objects so obtained are sequences of numbers, and as such are not the elements in the field $\mathbb{R}$ or $\mathbb{C}$. Instead, they are the representatives of Colombeau's generalized numbers. We give the exact definition of these "numbers".

Definition 2.3. Inserting $p \in M$ into $u \in \mathcal{G}(M)$ yields a well defined element of the ring of constants (also called generalized numbers) $\mathcal{K}$ (corresponding to $\mathbf{K}=\mathbb{R}$ resp. $\mathbb{C}$ ), defined as the set of moderate nets of numbers $\left(\left(r_{\varepsilon}\right)_{\varepsilon} \in \mathbf{K}^{(0,1]}\right.$ with $\left|r_{\varepsilon}\right|=O\left(\varepsilon^{-N}\right)$ for some $\left.N\right)$ modulo negligible nets $\left(\left|r_{\varepsilon}\right|=O\left(\varepsilon^{m}\right)\right.$ for each $\left.m\right)$;componentwise insertion of points of Minto elements of $\mathcal{G}(M)$ yields well-defined generalized numbers, i.e., elements of the ring of constants:

$$
\mathcal{K}=\mathcal{E}_{\mathbf{c}}(M) / \mathcal{N}_{\mathbf{c}}(M)
$$

(with $\mathrm{K}=\widetilde{\mathbb{R}}$ or $\mathcal{K}=\widetilde{\mathbb{C}}$ for $\mathbf{K}=\mathbb{R}$ or $\mathbf{K}=\mathbb{C}$ ), where

$$
\left\{\begin{array}{c}
\mathcal{E}_{\mathbf{c}}(M)=\left\{\left(r_{\epsilon}\right)_{\epsilon} \in \mathbf{K}^{I} \mid \exists n(n \in \mathbb{N})\left[\left|r_{\epsilon}\right|=O\left(\epsilon^{-n}\right) \text { as } \varepsilon \rightarrow 0\right]\right\} \\
\mathcal{N}_{\mathbf{c}}(M)=\left\{\left(r_{\epsilon}\right)_{\epsilon} \in \mathbf{K}^{I} \mid \forall m(m \in \mathbb{N})\left[\left|r_{\epsilon}\right|=O\left(\epsilon^{m}\right) \text { as } \varepsilon \rightarrow 0\right]\right\} \\
I=(0,1] .
\end{array}\right.
$$

Generalized functions on Mare characterized by their generalized point values, i.e., by their values on points in $\tilde{M}_{c}$, the space of equivalence classes of compactly supported nets $\left(p_{\varepsilon}\right)_{\varepsilon} \in M^{(0,1]}$ with respect to the relation $p_{\varepsilon} \sim p_{\varepsilon}^{\prime}: \Leftrightarrow d_{h}\left(p_{\varepsilon}, p_{\varepsilon}^{\prime}\right)=O\left(\varepsilon^{m}\right)$ for all $m$, where $d_{h}$ denotes the distance on Minduced by any Riemannian metric.

Definition 2.4. For $u \in \mathcal{G}(M)$ and $x_{0} \in M$, the point value of $u$ at the point $x_{0}, u\left(x_{0}\right)$, is defined as the class of $\left(u_{\varepsilon}\left(x_{0}\right)\right)_{\varepsilon}$ in $\mathcal{K}$. 
Definition 2.5. We say that an element $r \in \mathcal{K}$ is strictly nonzero if there exists a representative $\left(r_{\varepsilon}\right)_{\varepsilon}$ and a $q \in \mathbb{N}$ such that $\left|r_{\varepsilon}\right| \geqslant \varepsilon^{q}$ for $\varepsilon$ sufficiently small. If $r$ is strictly nonzero, then it is also invertible with the inverse $\left[\left(1 / r_{\varepsilon}\right)_{\varepsilon}\right]$. The converse is true as well. Treating the elements of Colombeau algebras as a generalization of classical functions, the question arises whether the definition of point values can be extended in such a way that each element is characterized by its values. Such an extension is indeed possible.

Definition 2.6. Let $\Omega$ be an open subset of $\mathbb{R}^{n}$. On a set $\hat{\Omega}$ :

$$
\left\{\begin{array}{c}
\hat{\Omega}=\left\{\left(x_{\varepsilon}\right)_{\varepsilon} \in \Omega^{I} \mid \exists p(p>0)\left[\left|x_{\varepsilon}\right|=O\left(\varepsilon^{p}\right)\right]\right\}= \\
\left\{\left(x_{\varepsilon}\right)_{\varepsilon} \in \Omega^{I} \mid \exists p(p>0) \exists \varepsilon_{0}\left(\varepsilon_{0}>0\right)\left[\left|x_{\varepsilon}\right| \leq \varepsilon^{p}, \text { for } 0<\varepsilon<\varepsilon_{0}\right]\right\}
\end{array}\right.
$$

we introduce an equivalence relation:

$$
\left(x_{\varepsilon}\right)_{\varepsilon} \sim\left(y_{\varepsilon}\right)_{\varepsilon} \Longleftrightarrow \forall q(q>0) \forall \varepsilon(\varepsilon>0)\left[\left|x_{\varepsilon}-y_{\varepsilon}\right| \leq \varepsilon^{q} \text {, for } 0<\varepsilon<\varepsilon_{0}\right]
$$

and denote by $\widetilde{\Omega}=\hat{\Omega} / \sim$ the set of generalized points. The set of points with compact support is

$$
\widetilde{\Omega}_{\mathbf{c}}=\left\{\widetilde{x}=\operatorname{cl}\left[\left(x_{\varepsilon}\right)_{\varepsilon}\right] \in \widetilde{\Omega} \mid \exists K(K \subset \Omega) \exists \varepsilon_{0}\left(\varepsilon_{0}>0\right)\left[x_{\varepsilon} \in K \text { for } 0<\varepsilon<\varepsilon_{0}\right]\right\}
$$

Definition 2.7. A generalized function $u \in \mathcal{G}(M)$ is called associated to zero, $u \approx 0$ on $\Omega \subseteq M$ in L.Schwartz sense if one (hence any) representative $\left(u_{\epsilon}\right)_{\epsilon}$ converges to zero weakly,i.e.

$$
w-\lim _{\epsilon \rightarrow 0} u_{\epsilon}=0
$$

We shall often write:

$$
u \underset{\text { Sch }}{\approx} 0 \text {. }
$$

The $\mathcal{G}(M)$-module of generalized sections in vector bundles-especially the space of generalized tensor fields $\mathcal{T}_{s}{ }^{r}(M)$-is defined along the same lines using analogous asymptotic estimates with respect to the norm induced by any Riemannian metric on the respective fibers. However, it is more convenient to use the following algebraic description of generalized tensor fields

$$
\mathcal{G}_{s}^{r}(M)=\mathcal{G}(M) \otimes \mathcal{T}_{s}^{r}(M),
$$

where $\mathcal{T}_{s}{ }^{r}(M)$ denotes the space of smooth tensor fields and the tensor product is taken over the module $C^{\infty}(M)$. Hence generalized tensor fields are just given by classical ones with generalized coefficient functions. Many concepts of classical tensor analysis carry over to the generalized setting [1],[2] in particular Lie derivatives with respect to both classical and generalized vector fields, Lie brackets, exterior algebra, etc. Moreover, generalized tensor fields may also be viewed as $\mathcal{G}(M)$ multilinear maps taking generalized vector and covector fields to generalized functions, i.e., as $\mathcal{G}(M)$-modules we have

$$
\mathcal{G}_{s}^{r}(M) \cong L_{(M)}\left(\mathcal{G}_{1}^{0}(M)^{r}, \mathcal{G}_{0}^{1}(M)^{s} ; \mathcal{G}(M)\right) .
$$

In particular a generalized metric is defined to be a symmetric, generalized $(0,2)$-tensor field $g_{a b}=$ $\left[\left(\left(g_{\epsilon}\right)_{a b}\right)_{\epsilon}\right]$ (with its index independent of Eand) whose determinant $\operatorname{det}\left(g_{a b}\right)$ is invertible in $\mathcal{G}(M)$. The latter condition is equivalent to the following notion called strictly nonzero on compact sets: for any representative $\operatorname{det}\left(\left(g_{\epsilon}\right)_{a b}\right)_{\epsilon}$ of $\operatorname{det}\left(g_{a b}\right)$ we have $\forall K \subset M \exists m \in \mathbb{N}\left[\inf _{p \in K}\left|\operatorname{det}\left(g_{a b}(\epsilon)\right)\right| \geq \epsilon^{m}\right]$ for all $\epsilon$ small enough. This notion captures the intuitive idea of a generalized metric to be a sequence of classical metrics approaching a singular limit in the following sense: $g_{a b}$ is a generalized metric iff (on every relatively compact open subset $V$ of $M$ ) there exists a representative $\left(\left(g_{\epsilon}\right)_{a b}\right)_{\epsilon}$ of $g_{a b}$ such that for fixed $\epsilon$ (small enough) $\left(g_{\epsilon}\right)_{a b}=g_{a b}(\epsilon)$ (resp. $\left.\left.\left(g_{\epsilon}\right)_{a b}\right|_{V}\right)$ is a classical pseudo-Riemannian metric and $\operatorname{det}\left(g_{a b}\right)$ is invertible in the algebra of generalized functions. A generalized metric induces a $\mathcal{G}(M)$-linear isomorphism from $\mathcal{G}_{0}^{1}(M)$ to $\mathcal{G}_{1}^{0}(M)$ and the inverse metric $g^{a b} \triangleq\left[\left(g_{a b}^{-1}(\epsilon)\right)_{\epsilon}\right]$ 
is a well defined element of $\mathcal{G}_{0}^{2}(M)$ (i.e., independent of the representative $\left.\left(\left(g_{\epsilon}\right)_{a b}\right)_{\epsilon}\right)$. Also the generalized Levi-Civita connection as well as the generalized Riemann-, Ricci- and Einstein tensor of a generalized metric are defined simply by the usual coordinate formulae on the level of representatives.

\subsection{Generalized Colombeau Calculus}

We briefly recall the basic generalized Colombeau construction. Colombeau supergeneralized functions on $\Omega \subseteq \mathbb{R}^{n}$, where $\operatorname{dim}(\Omega)=n$ are defined as equivalence classes $u=\left[\left(u_{\varepsilon}\right)_{\varepsilon}\right]$ of nets of smooth functions $u_{\varepsilon} \in C^{\infty}(\Omega \backslash \Sigma)$, where $\operatorname{dim}(\Sigma)<n$ (regularizations) subjected to asymptotic norm conditions with respect to $\varepsilon \in(0,1]$ for their derivatives on compact sets.

The basic idea of generalized Colombeau's theory of nonlinear supergeneralized functions [1],[2, is regularization by sequences (nets) of smooth functions and the use of asymptotic estimates in terms of a regularization parameter $\varepsilon$. Let $\left(u_{\varepsilon}\right)_{\varepsilon i n(0,1]}$ with $u_{\varepsilon}$ such that: (i) $u_{\varepsilon} \in \mathcal{C}^{\infty}(M \backslash \Sigma)$ and (ii) $u_{\varepsilon} \in D^{\prime}(M)$, for all $\varepsilon \in(0,1]$, where $M$ is a separable, smooth orientable Hausdorff manifold of dimension $n$.

Definition 2.8. The supergeneralized Colombeau's algebra $\widetilde{\mathcal{G}}=\widetilde{\mathcal{G}}(M, \Sigma)$ of supergeneralized functions on $M$, where $\Sigma$ subset $M, \operatorname{dim}(M)=n, \operatorname{dim}(\Sigma)<n$,is defined as the quotient:

$$
\widetilde{\mathcal{G}}(M, \Sigma) \triangleq \mathcal{E}_{M}(M, \Sigma) / \mathcal{N}(M, \Sigma)
$$

of the space $\mathcal{E}_{M}(M, \Sigma)$ of sequences of moderate growth modulo the space $\mathcal{N}(M, \Sigma)$ of negligible sequences More precisely the notions of moderateness resp. negligibility are defined by the following asymptotic estimates (where $\mathfrak{X}(M \backslash \Sigma)$ denoting the space of smooth vector fields on $M \backslash \Sigma$

$$
\begin{aligned}
& \mathcal{E}_{M}(M, \Sigma) \triangleq\left\{\left(u_{\varepsilon}\right)_{\varepsilon} \mid \forall K(K \varsubsetneqq M \backslash \Sigma) \forall k(k \in \mathbb{N}) \exists N(N \in \mathbb{N})\right. \\
& \forall \xi_{1}, \ldots, \xi_{k}\left(\xi_{1}, \ldots, \xi_{k} \in \mathfrak{X}(M \backslash \Sigma)\right)\left[\sup _{p \in K}\left|L_{\xi_{1}} \ldots L_{\xi_{k}} u_{\varepsilon}(p)\right|=O\left(\varepsilon^{-N}\right), \varepsilon \rightarrow 0\right] \& \\
& \forall K(K \varsubsetneqq M) \forall k(k \in \mathbb{N}) \exists N(N \in \mathbb{N}) \forall\left(f \in \mathcal{C}^{\infty}(M)\right) \forall \xi_{1}, \ldots, \xi_{k}\left(\xi_{1}, \ldots, \xi_{k} \in \mathfrak{X}(M)\right) \\
& \left.\left[\left\|L_{\xi_{1}}^{w} \ldots L_{\xi_{k}}^{w} u_{\varepsilon}\right\|=\left(\sup _{f \in \mathcal{C}^{\infty}(M)}\left|L_{\xi_{1}}^{w} \ldots L_{\xi_{k}}^{w} u_{\varepsilon}(f)\right|\right)=O\left(\varepsilon^{-N}\right), \varepsilon \rightarrow 0\right]\right\}, \\
& \mathcal{N}(M, \Sigma) \triangleq\left\{\left(u_{\varepsilon}\right)_{\varepsilon} \mid \forall K(K \varsubsetneqq M \backslash \Sigma), \forall k\left(k \in \mathbb{N}_{0}\right) \forall q(q \in N)\right. \\
& \left.\forall \xi_{1}, \ldots, \xi_{k}\left(\xi_{1}, \ldots, \xi_{k} \in \mathfrak{X}(M \backslash \Sigma)\right)\left[\sup _{p \in K}\left|L_{\xi_{1}} \ldots L_{\xi_{k}} u_{\varepsilon}(p)\right|=O\left(\varepsilon^{q}\right), \varepsilon \rightarrow 0\right]\right\} \& \\
& \forall K(K \varsubsetneqq M) \forall k(k \in \mathbb{N}) \exists N(N \in \mathbb{N}) \forall\left(f \in \mathcal{C}^{\infty}(M)\right) \forall \xi_{1}, \ldots, \xi_{k}\left(\xi_{1}, \ldots, \xi_{k} \in \mathfrak{X}(M)\right) \\
& \left.\left[\left\|L_{\xi_{1}}^{w} \ldots L_{\xi_{k}}^{w} u_{\varepsilon}\right\|=\left(\sup _{f \in \mathcal{C}^{\infty}(M)}\left|L_{\xi_{1}}^{w} \ldots L_{\xi_{k}}^{w} u_{\varepsilon}(f)\right|\right)=O\left(\varepsilon^{q}\right), \varepsilon \rightarrow 0\right]\right\},
\end{aligned}
$$

where $\mathrm{L}_{\xi_{k}}^{w}$ denoting the weak Lie derivative in L. Schwartz sense. In the definition the Landau symbol $a_{\varepsilon}=O(\psi(\varepsilon))$ appears, having the following meaning:

$$
\exists C(C>0) \exists \varepsilon_{0}\left(\varepsilon_{0} \in(0,1]\right) \forall \varepsilon\left(\varepsilon<\varepsilon_{0}\right)\left[a_{\varepsilon} \leq C \psi(\varepsilon)\right] .
$$

Definition 2.9. Elements of $\widetilde{\mathcal{G}}(M, \Sigma)$ are denoted by:

$$
u=\operatorname{cl}\left[\left(u_{\varepsilon}\right)_{\varepsilon}\right] \triangleq\left(u_{\varepsilon}\right)_{\varepsilon}+\mathcal{N}(M, \Sigma) \text {. }
$$

Remark 2.4 With componentwise operations $(\cdot, \pm) \widetilde{\mathcal{G}}(M, \Sigma)$ is a fine sheaf of differential algebras with respect to the Lie derivative defined by $L_{\xi} u \triangleq \operatorname{cl}\left[\left(L_{\xi} u_{\varepsilon}\right)_{\varepsilon}\right]$.

The spaces of moderate resp. negligible sequences and hence the algebra itself may be characterized locally, i.e., $u \in \widetilde{\mathcal{G}}(M, \Sigma)$ iff $u \circ \psi_{\alpha} \in \widetilde{\mathcal{G}}\left(\psi_{\alpha}\left(V_{\alpha}\right)\right)$ for all charts $\left(V_{\alpha}, \psi_{\alpha}\right)$, where on the open set $\psi_{\alpha}\left(V_{\alpha}\right) \subset \mathbb{R}^{n}$ in the respective estimates Lie derivatives are replaced by partial derivatives. 
Remark 2.5 Smooth functions $\mathrm{f} \in \mathcal{C}^{\infty}(M \backslash \Sigma)$ are embedded into $\widetilde{\mathcal{G}}(M, \Sigma)$ simply by the "constant" embedding $\sigma$, i.e., $\sigma(f)=\operatorname{cl}\left[(f)_{\varepsilon}\right]$, hence $\mathcal{C}^{\infty}(M \backslash \Sigma)$ is a faithful subalgebra of $\widetilde{\mathcal{G}}(M, \Sigma)$.

\subsubsection{Point values of a supergeneralized functions on $M$. supergenera- lized numbers}

Within the classical distribution theory, distributions cannot be characterized by their point values in any way similar to classical functions. On the other hand, there is a very natural and direct way of obtaining the point values of the elements of Colombeau's algebra: points are simply inserted into representatives. The objects so obtained are sequences of numbers, and as such are not the elements in the field $\mathbb{R}$ or $\mathbb{C}$. Instead, they are the representatives of Colombeau's generalized numbers. We give the exact definition of these "numbers".

Definition 2.10. Inserting $\mathrm{p} \in M$ into $u \in \widetilde{\mathcal{G}}(M, \Sigma)$ yields a well defined element of the ring of constants (also called generalized numbers) $\widetilde{\mathcal{K}}$ (corresponding to $\mathbf{K}=\mathbb{R}$ resp. $\mathbb{C}$ ), defined as the set of moderate nets of numbers $\left(\left(r_{\varepsilon}\right)_{\varepsilon} \in \mathbf{K}^{(0,1]}\right.$ with $\left|r_{\varepsilon}\right|=O\left(\varepsilon^{-N}\right)$ for some $\left.N\right)$ modulo negligible nets $\left(\left|r_{\varepsilon}\right|=O\left(\varepsilon^{m}\right)\right.$ for each $\left.m\right)$; componentwise insertion of points of Minto elements of $\widetilde{\mathcal{G}}(M, \Sigma)$ yields well-defined generalized numbers, i.e., elements of the ring of constants:

$$
\widetilde{\mathcal{K}}_{\Sigma}=\mathcal{E}_{\mathbf{c}}(M, \Sigma) / \mathcal{N}_{\mathbf{c}}(M, \Sigma)
$$

(with $\widetilde{\mathcal{K}}_{\Sigma}=\widetilde{\mathbb{R}}_{\Sigma}$ or $\mathcal{K}=\widetilde{\mathbb{C}}_{\Sigma}$ for $\mathbf{K}=\mathbb{R}$ or $\mathbf{K}=\mathbb{C}$ ), where

$$
\left\{\begin{array}{c}
\mathcal{E}_{\mathbf{c}}(M, \Sigma)=\left\{\left(r_{\epsilon}\right)_{\epsilon} \in \mathbf{K}^{I} \mid \exists n(n \in \mathbb{N})\left[\left|r_{\varepsilon}\right|=O\left(\varepsilon^{-n}\right) \text { as } \varepsilon \rightarrow 0\right]\right\} \\
\mathcal{N}_{\mathbf{c}}(M, \Sigma)=\left\{\left(r_{\epsilon}\right)_{\epsilon} \in \mathbf{K}^{I} \mid \forall m(m \in \mathbb{N})\left[\left|r_{\varepsilon}\right|=O\left(\varepsilon^{m}\right) \text { as } \varepsilon \rightarrow 0\right]\right\} \\
I=(0,1]
\end{array}\right.
$$

Supergeneralized functions on $M$ are characterized by their generalized point values, i.e., by their values on points in $\tilde{M}_{c}$, the space of equivalence classes of compactly supported nets $\left(p_{\varepsilon}\right)_{\varepsilon} \in$ $(M \backslash \Sigma)^{(0,1]}$ with respect to the relation $p_{\varepsilon} \sim p_{\varepsilon}^{\prime}: \Leftrightarrow d_{h}\left(p_{\varepsilon}, p_{\varepsilon}^{\prime}\right)=O\left(\varepsilon^{m}\right)$ for all $m$, where $d_{h}$ denotes the distance on $M \backslash \Sigma$ induced by any Riemannian metric.

Definition 2.11. For $\mathrm{u} \in \widetilde{\mathcal{G}}(M, \Sigma)$ and $x_{0} \in M$, the point value of $u$ at the point $x_{0}, u\left(x_{0}\right)$, is defined as the class of $\left(u_{\varepsilon}\left(x_{0}\right)\right)_{\varepsilon}$ in $\widetilde{\mathcal{K}}$.

Definition 2.12. We say that an element $\mathrm{r} \in \widetilde{\mathcal{K}}$ is strictly nonzero if there exists a representative $\left(r_{\varepsilon}\right)_{\varepsilon}$ and a $q \in \mathrm{N}$ such that $-\mathrm{r}_{\varepsilon} \mid \geqslant \varepsilon^{q}$ for $\varepsilon$ sufficiently small. If $r$ is strictly nonzero, then it is also invertible with the inverse $\left[\left(1 / r_{\varepsilon}\right)_{\varepsilon}\right]$. The converse is true as well. Treating the elements of Colombeau algebras as a generalization of classical functions, the question arises whether the definition of point values can be extended in such a way that each element is characterized by its values. Such an extension is indeed possible.

Definition 2.13. Let $\Omega$ be an open subset of $\mathbb{R}^{n} \backslash \Sigma$. On a set $\hat{\Omega}_{\Sigma}$ :

$$
\left\{\begin{array}{c}
\hat{\Omega}_{\Sigma}=\left\{\left(x_{\varepsilon}\right)_{\varepsilon} \in(\Omega \backslash \Sigma)^{I} \mid \exists p(p>0)\left[\left|x_{\varepsilon}\right|=O\left(\varepsilon^{p}\right)\right]\right\}= \\
\left\{\left(x_{\varepsilon}\right)_{\varepsilon} \in(\Omega \backslash \Sigma)^{I} \mid \exists p(p>0) \exists \varepsilon_{0}\left(\varepsilon_{0}>0\right)\left[\left|x_{\varepsilon}\right| \leq \varepsilon^{p}, \text { for } 0<\varepsilon<\varepsilon_{0}\right]\right\}
\end{array}\right.
$$

we introduce an equivalence relation: 


$$
\left(x_{\varepsilon}\right)_{\varepsilon} \sim\left(y_{\varepsilon}\right)_{\varepsilon} \Longleftrightarrow \forall q(q>0) \forall \varepsilon(\varepsilon>0)\left[\left|x_{\varepsilon}-y_{\varepsilon}\right| \leq \varepsilon^{q}, \text { for } 0<\varepsilon<\varepsilon_{0}\right]
$$

and denote by $\widetilde{\Omega}_{\Sigma}=\hat{\Omega}_{\Sigma} / \sim$ the set of supergeneralized points. The set of points with compact support is

$$
\widetilde{\Omega}_{\Sigma, \mathbf{c}}=\left\{\widetilde{x}=\operatorname{cl}\left[\left(x_{\varepsilon}\right)_{\varepsilon}\right] \in \widetilde{\Omega}_{\Sigma} \mid \exists K(K \subset \Omega \backslash \Sigma) \exists \varepsilon_{0}\left(\varepsilon_{0}>0\right)\left[x_{\varepsilon} \in K \text { for } 0<\varepsilon<\varepsilon_{0}\right]\right\}
$$

Definition 2.14. A supergeneralized function $\mathrm{u} \in \widetilde{\mathcal{G}}(M, \Sigma)$ is called associated to zero, $u \approx 0$ on $\Omega \subseteq M$ in L. Schwartz's sense if one (hence any) representative $\left(u_{\varepsilon}\right)_{\varepsilon}$ converges to zero weakly,i.e.

$w-\lim _{\varepsilon \rightarrow 0} u_{\varepsilon}=0$

We shall often write:

$u \underset{\text { Sch }}{\approx} 0$.

Definition 2.15. The $\widetilde{\mathcal{G}}(M, \Sigma)$-module of supergeneralized sections in vector bundles- especially the space of generalized tensor fields $\mathcal{T}_{s}^{r}(M \backslash \Sigma)$-is defined along the same lines using analogous asymptotic estimates with respect to the norm induced by any Riemannian metric on the respective fibers. However, it is more convenient to use the following algebraic description of generalized tensor fields

$$
\widetilde{\mathcal{G}}_{s}^{r}(M, \Sigma)=\widetilde{\mathcal{G}}(M, \Sigma) \otimes \mathcal{T}_{s}^{r}(M \backslash \Sigma),
$$

where $\mathrm{T}_{s}{ }^{r}(M \backslash \Sigma)$ denotes the space of smooth tensor fields and the tensor product is taken over the module $C^{\infty}(M \backslash \Sigma)$. Hence generalized tensor fields are just given by classical ones with generalized coefficient functions. Many concepts of classical tensor analysis carry over to the generalized setting, in particular Lie derivatives with respect to both classical and generalized vector fields, Lie brackets, exterior algebra, etc. Moreover, generalized tensor fields may also be viewed as $\widetilde{\mathcal{G}}(M, \Sigma)$-multilinear maps taking generalized vector and covector fields to generalized functions, i.e., as $\widetilde{\mathcal{G}}(M, \Sigma)$-modules we have

$$
\widetilde{\mathcal{G}}_{s}^{r}(M, \Sigma) \cong L_{(M)}\left(\widetilde{\mathcal{G}}_{1}^{0}(M, \Sigma)^{r}, \widetilde{\mathcal{G}}_{0}^{1}(M, \Sigma)^{s} ; \widetilde{\mathcal{G}}(M, \Sigma)\right) .
$$

In particular a supergeneralized metric is defined to be a symmetric, supergeneralized (0,2)-tensor field $g_{a b}=\left[\left(\left(g_{\varepsilon}\right)_{a b}\right)_{\varepsilon}\right]$ (with its index independent of $\varepsilon$ and) whose determinant $\operatorname{det}\left(g_{a b}\right)$ is invertible in $\widetilde{\mathcal{G}}(M \backslash \Sigma)$. The latter condition is equivalent to the following notion called strictly nonzero on compact sets: for any representative $\operatorname{det}\left(\left(g_{\varepsilon}\right)_{a b}\right)_{\varepsilon}$ of $\operatorname{det}\left(g_{a b}\right)$ we have $\forall K \subset M \backslash \Sigma \exists m \in$ $\mathbb{N}\left[\inf _{p \in K}\left|\operatorname{det}\left(g_{a b}(\varepsilon)\right)\right| \geq \varepsilon^{q}\right]$ for all $\varepsilon$ small enough. This notion captures the intuitive idea of a generalized metric to be a sequence of classical metrics approaching a singular limit in the following sense: $g_{a b}$ is a generalized metric iff (on every relatively compact open subset $V$ of $M$ ) there exists a representative $\left(\left(g_{\varepsilon}\right)_{a b}\right)_{\varepsilon}$ of $g_{a b}$ such that for fixed $\varepsilon$ (small enough) $\left(g_{\varepsilon}\right)_{a b}=g_{a b}(\varepsilon)$ (resp. $\left.\left.\left(g_{\varepsilon}\right)_{a b}\right|_{V}\right)$ is a classical pseudo-Riemannian metric and $\operatorname{det}\left(g_{a b}\right)$ is invertible in the algebra of generalized functions. A generalized metric induces a $\widetilde{\mathcal{G}}(M, \Sigma)$-linear isomorphism from $\widetilde{\mathcal{G}}_{0}^{1}(M, \Sigma)$ to $\widetilde{\mathcal{G}}_{1}^{0}(M, \Sigma)$ and the inverse metric $g^{a b} \triangleq\left[\left(g_{a b}^{-1}(\varepsilon)\right)_{\varepsilon}\right]$ is a well defined element of $\widetilde{\mathcal{G}}_{0}^{2}(M, \Sigma)$ (i.e., independent of the representative $\left.\left(\left(g_{\varepsilon}\right)_{a b}\right)_{\varepsilon}\right)$. Also the supergeneralized Levi-Civita connection as well as the supergeneralized Riemann, Ricci and Einstein tensor of a supergeneralized metric are defined simply by the usual coordinate formulae on the level of representatives.

\subsection{Distributional general relativity}

We briefly summarize the basics of distributional general relativity, as a preliminary to latter discussion. In the classical theory of gravitation one is led to consider the Einstein field equations 
which are, in general, quasilinear partial differential equations involving second order derivatives for the metric tensor. Hence, continuity of the first fundamental form is expected and at most, discontinuities in the second fundamental form, the coordinate independent statements appropriate to consider 3-surfaces of discontinuity in the spacetime manifolfd of General Relativity.

In standard general relativity, the space-time is assumed to be a four-dimensional differentiable manifold $M$ endowed with the Lorentzian metric $d s^{2}=g_{\mu \nu} d x^{\mu} d x^{\nu}(\mu, \nu=0,1,2,3)$. At each point $p$ of space-time $M$, the metric can be diagonalized as $d s_{p}^{2}=\eta_{\mu \nu}\left(d X^{\mu}\right)_{p}\left(d X^{\nu}\right)_{p}$ with $\eta_{\mu \nu} \triangleq(-1,1,1,1)$, by choosing the coordinate system $\left\{X^{\mu} ; \mu=0,1,2,3\right\}$ appropriately.

In superdistributional general relativity the space-time is assumed to be a four-dimensional differentiable manifold $M \backslash \Sigma$, where $\operatorname{dim}(M)=4, \operatorname{dim}(\Sigma) \leqslant 3$ endowed with the Lorentzian supergeneralized metric

$$
\left.\left(d s_{\epsilon}^{2}\right)_{\epsilon}=\left(g_{\mu \nu}(\epsilon) d x^{\mu} d x^{\nu}\right)_{\epsilon} ; \mu, \nu=0,1,2,3\right) .
$$

At each point $\mathrm{p} \in M \backslash \Sigma$, the metric can be diagonalized as

$$
\left(d s_{p}^{2}(\epsilon)\right)_{\epsilon}=\left(\eta_{\mu \nu}\left(d X_{\epsilon}^{\mu}\right)_{p}\left(d X_{\epsilon}^{\nu}\right)_{p}\right)_{\epsilon} \text { with } \eta_{\mu \nu} \triangleq(-1,1,1,1),
$$

by choosing the generalized coordinate system $\left\{\left(X_{\epsilon}^{\mu}\right)_{\epsilon} ; \mu=0,1,2,3\right\}$ appropriately.The classical smooth curvature tensor is given by

$$
R_{\sigma \mu \nu}^{\rho} \triangleq \partial_{\mu}\left\{\frac{\rho}{\sigma \nu}\right\}-\partial_{\nu}\left\{\frac{\rho}{\sigma \mu}\right\}+\left\{\frac{\rho}{\lambda \mu}\right\}\left\{\frac{\lambda}{\sigma \nu}\right\}-\left\{\frac{\rho}{\lambda \nu}\right\}\left\{\frac{\lambda}{\sigma \mu}\right\}
$$

with $\left\{\frac{\rho}{\sigma \nu}\right\}$ being the smooth Christoffel symbol. The supergeneralized nonsmooth curvature tensor is given by

$$
\left\{\begin{array}{c}
\left(R_{\sigma \mu \nu}^{\rho}(\epsilon)\right)_{\epsilon} \triangleq \partial_{\mu}\left(\left\{\frac{\rho}{\sigma \nu}\right\}_{\epsilon}\right)_{\epsilon}-\partial_{\nu}\left(\left\{\frac{\rho}{\sigma \mu}\right\}_{\epsilon}\right)_{\epsilon}+\left(\left\{\frac{\rho}{\lambda \mu}\right\}_{\epsilon}\right)_{\epsilon}\left(\left\{\frac{\lambda}{\sigma \nu}\right\}_{\epsilon}\right)_{\epsilon}- \\
-\left(\left\{\frac{\rho}{\lambda \nu}\right\}_{\epsilon}\right)_{\epsilon}\left(\left\{\frac{\lambda}{\sigma \mu}\right\}_{\epsilon}\right)_{\epsilon}
\end{array}\right.
$$

with $\left(\left\{\frac{\rho}{\sigma \nu}\right\}_{\epsilon}\right)_{\epsilon}$ being the supergeneralized Christoffel symbol. The fundamental classical action integral $\mathbb{I}$ is

$$
\mathbb{I}=\frac{1}{c}\left(\overline{\mathbb{L}}_{G}+\mathbb{L}_{M}\right) d^{4} x
$$

where $\mathbb{L}_{M}$ is the Lagrangian density of a gravitational source and $\overline{\mathbb{L}}_{G}$ is the gravitational Lagrangian density given by

$$
\overline{\mathbb{L}}_{G}=\frac{1}{2 \kappa} \mathbb{G} \text {. }
$$

Here $\kappa$ is the Einstein gravitational constant $\kappa=8 \pi G / c^{4}$ and $\mathbb{G}$ is defined by

$$
\mathbb{G}=\sqrt{-g} g^{\mu \nu}\left(\left\{\frac{\lambda}{\mu \rho}\right\}\left\{\frac{\rho}{\nu \lambda}\right\}-\left\{\frac{\lambda}{\mu \nu}\right\}\left\{\frac{\rho}{\lambda \rho}\right\}\right)
$$

with $g=\operatorname{det}\left(g_{\mu \nu}\right)$. There exists the relation

$$
\sqrt{-g} R=\mathbb{G}+\partial_{\mu} \mathbb{D}^{\mu},
$$

with

$$
\mathbb{D}^{\mu}=-\sqrt{-g}\left(g^{\mu \nu}\left\{\frac{\lambda}{\nu \lambda}\right\}-g^{\nu \lambda}\left\{\frac{\mu}{\nu \lambda}\right\}\right) .
$$

Thus the supergeneralized fundamental action integral $\left(\mathbb{I}_{\epsilon}\right)_{\epsilon}$ is

$$
\left(\mathbb{I}_{\epsilon}\right)_{\epsilon}=\frac{1}{c} \int\left(\left(\overline{\mathbb{L}}_{G}(\epsilon)\right)_{\epsilon}+\left(\mathbb{L}_{M}(\epsilon)\right)_{\epsilon}\right) d^{4} x,
$$

where $\left(\mathbb{L}_{M}(\epsilon)\right)_{\epsilon}$ is the supergeneralized Lagrangian density of a gravitational source and $\left(\overline{\mathbb{L}}_{G}(\epsilon)\right)_{\epsilon}$ 
is the supergeneralized gravitational Lagrangian density given by

$$
\left(\overline{\mathbb{L}}_{G}(\epsilon)\right)_{\epsilon}=\frac{1}{2 \kappa}\left(\mathbb{G}_{\epsilon}\right)_{\epsilon} .
$$

Here $\kappa$ is the Einstein gravitational constant $\kappa=8 \pi G / c^{4}$ and $\left(\mathbb{G}_{\epsilon}\right)_{\epsilon}$ is defined by

$$
\left(\mathbb{G}_{\epsilon}\right)_{\epsilon}=\sqrt{-\left(g_{\epsilon}\right)_{\epsilon}}\left(g_{\epsilon}^{\mu \nu}\right)_{\epsilon}\left(\left(\left\{\frac{\lambda}{\mu \rho}\right\}_{\epsilon}\right)\left(\left\{\frac{\rho}{\nu \lambda}\right\}_{\epsilon}\right)-\left(\left\{\frac{\lambda}{\mu \nu}\right\}_{\epsilon}\right)\left(\left\{\frac{\rho}{\lambda \rho}\right\}_{\epsilon}\right)\right)
$$

with $g_{\epsilon}=\operatorname{det}\left[\left(g_{\mu \nu}(\epsilon)\right)_{\epsilon}\right]$. There exists the relation

$$
\sqrt{-\left(g_{\epsilon}\right)_{\epsilon}}\left(\mathbf{R}_{\epsilon}\right)_{\epsilon}=\left(\mathbb{G}_{\epsilon}\right)_{\epsilon}+\partial_{\mu}\left(\mathbb{D}_{\epsilon}^{\mu}\right)_{\epsilon},
$$

with

$$
\left(\mathbb{D}_{\epsilon}^{\mu}\right)_{\epsilon}=-\sqrt{-\left(g_{\epsilon}\right)_{\epsilon}}\left(\left(g_{\epsilon}^{\mu \nu}\right)_{\epsilon}\left(\left\{\frac{\lambda}{\nu \lambda}\right\}_{\epsilon}\right)_{\epsilon}-\left(g_{\epsilon}^{\nu \lambda}\right)_{\epsilon}\left(\left\{\frac{\mu}{\nu \lambda}\right\}_{\epsilon}\right)_{\epsilon}\right) .
$$

Also, we have defined the classical scalar curvature by

$$
\mathbf{R}=R^{\mu}{ }_{\mu}
$$

with the smooth Ricci tensor

$$
R_{\mu \nu}=R_{\mu \lambda \nu}^{\lambda} \text {. }
$$

From the action $\mathbb{I}$, the classical Einstein equation

$$
G_{\mu}{ }^{\nu}=R_{\mu}{ }^{\nu}-\frac{1}{2} \delta_{\mu}{ }^{\nu} R=\kappa T_{\mu}{ }^{\nu}
$$

follows, where $T_{\mu}{ }^{\nu}$ is defined by

$$
T_{\mu}{ }^{\nu}=\frac{\widetilde{\mathbf{T}}_{\mu}{ }^{\nu}}{\sqrt{-g}}
$$

with

$$
\widetilde{\mathbf{T}}_{\mu}{ }^{\nu} \triangleq 2 g_{\mu \lambda} \frac{\delta \mathbb{L}_{M}}{\delta g_{\lambda \nu}}
$$

being the energy-momentum density of the classical gravity source. Thus we have defined the supergeneralized scalar curvature by

$$
\left(\mathbf{R}_{\epsilon}\right)_{\epsilon}=\left(R_{\mu}^{\mu}(\epsilon)\right)_{\epsilon}
$$

with the supergeneralized Ricci tensor

$$
\left(\mathbf{R}_{\mu \nu}(\epsilon)\right)_{\epsilon}=\left(R^{\lambda}{ }_{\mu \lambda \nu}(\epsilon)\right)_{\epsilon} .
$$

From the action $\left(\mathbb{I}_{\epsilon}\right)_{\epsilon}$, the supergeneralized Einstein equation

$$
\left(G_{\mu}{ }^{\nu}(\epsilon)\right)_{\epsilon}=\left(R_{\mu}^{\nu}(\epsilon)\right)_{\epsilon}-\frac{1}{2} \delta_{\mu}^{\nu}\left(\mathbf{R}_{\epsilon}\right)_{\epsilon}=\kappa\left(T_{\mu}{ }^{\nu}(\epsilon)\right)_{\epsilon},
$$

follows, where $\left(T_{\mu}^{\nu}(\epsilon)\right)_{\epsilon}$ is defined by

$$
\left(T_{\mu}^{\nu}(\epsilon)\right)_{\epsilon}=\frac{\left(\widetilde{\mathbf{T}}_{\mu}{ }^{\nu}(\epsilon)\right)_{\epsilon}}{\sqrt{-\left(g_{\epsilon}\right)_{\epsilon}}}
$$

with

$$
\left(\widetilde{\mathbf{T}}_{\mu}^{\nu}(\epsilon)\right)_{\epsilon} \triangleq 2\left(g_{\mu \lambda}(\epsilon)\right)_{\epsilon} \frac{\delta\left(\mathbb{L}_{M}(\epsilon)\right)_{\epsilon}}{\delta\left(g_{\lambda \nu}(\epsilon)\right)_{\epsilon}}
$$


being the supergeneralized energy-momentum density of the supergeneralized gravity source.The classical energy-momentum pseudo-tensor density $\widetilde{\mathbf{t}}_{\mu}{ }^{\nu}$ of the gravitational field is defined by

$$
\widetilde{\mathbf{t}}_{\mu}{ }^{\nu}=\delta_{\mu}{ }^{\nu} \overline{\mathbb{L}}_{G}-\frac{\partial \overline{\mathbb{L}}_{G}}{\partial g_{\sigma \tau, \nu}} g_{\sigma \tau, \mu}
$$

with $\mathrm{g}_{\sigma \tau, \nu}=\partial g_{\sigma \tau} / \partial x^{\nu}$. The supergeneralized energy-momentum pseudo-tensor density $\widetilde{\mathbf{t}}_{\mu}{ }^{\nu}$ of the gravitational field is defined by

$$
\left(\widetilde{\mathbf{t}}_{\mu}^{\nu}(\epsilon)\right)_{\epsilon}=\delta_{\mu}^{\nu}\left(\overline{\mathbb{L}}_{G}(\epsilon)\right)_{\epsilon}-\left(\frac{\partial \overline{\mathbb{L}}_{G}(\epsilon)}{\partial g_{\sigma \tau, \nu}(\epsilon)}\right)_{\epsilon}\left(g_{\sigma \tau, \mu}(\epsilon)\right)_{\epsilon}
$$

with $\left(g_{\sigma \tau, \nu}(\epsilon)\right)_{\epsilon}=\left(\partial g_{\sigma \tau}(\epsilon) / \partial x^{\nu}\right)_{\epsilon}$.

\section{Distributional Schwarzschild Geometry from Non- smooth Regularization via Horizon}

\subsection{Calculation of the stress-tensor by using nonsmooth regulariza- tion via horizon}

In this section we leave the neighborhood of the singularity at the origin and turn to the singularity at the horizon. The question we are aiming at is the following: using distributional geometry (thus without leaving Schwarzschild coordinates), is it possible to show that the horizon singularity of the Schwarzschild metric is not merely only a coordinate singularity.

In order to investigate this issue we calculate the distributional curvature at horizon in Schwarzschild coordinates. In the usual Schwarzschild coordinates $(t, r, \theta, \phi), r \neq 2 m$ the Schwarzschild metric (1.12) takes the form above horizon $r>2 m$ and below horizon $r<2 m$ correspondingly

$$
\left\{\begin{array}{c}
\text { above horizon } r>2 m: \\
d s^{+2}=h^{+}(r) d t^{2}-\left[h^{+}(r)\right]^{-1} d r^{2}+r^{2} d \Omega^{2} \\
h^{+}(r)=-1+\frac{2 m}{r}=-\frac{r-2 m}{r} \\
\text { below horizon } r<2 m ! \\
d s^{-2}=h^{-}(r) d t^{2}-h^{-}(r)^{-1} d r^{2}+r^{2} d \Omega^{2} \\
h^{-}(r)=-1+\frac{2 m}{r}=\frac{2 m-r}{r}
\end{array}\right.
$$

Remark 3.1 Following the above discussion we consider the metric coefficients $h^{+}(r),\left[h^{+}(r)\right]^{-1}$ $h^{-}(r)$, and $\left[h^{-}(r)\right]^{-1}$ as an element of $\mathcal{D}^{\prime}\left(\mathbb{R}^{3}\right)$ and embed it into $\left(\mathcal{G}\left(\mathbb{R}^{3}\right)\right)$ by replacements above horizon $r \geqslant 2 m$ and below horizon $r \leqslant 2 m$ correspondingly 
Remark 3.2 Note that, accordingly, we have fixed the differentiable structure of the manifold: the usual Scharzschild coordinates and the Cartesian coordinates associated with the spherical Schwarzschild coordinates in (3.1) are extended on $r=2 m$ through the horizon. Therefore we have above horizon $r \geqslant 2 m$ and below horizon $r \leqslant 2 m$ correspondingly

$$
h(r)=\left\{\begin{array}{c}
-\frac{r-2 m}{r} \text { if } r \geqslant 2 m \\
0 \text { if } r \leqslant 2 m
\end{array}\right\} \longmapsto\left(h_{\epsilon}^{+}(r)\right)_{\epsilon}=\left(-\frac{\sqrt{(r-2 m)^{2}+\epsilon^{2}}}{r}\right)_{\epsilon},
$$

where $\left(h_{\epsilon}^{+}(r)\right)_{\epsilon} \in \widetilde{\mathcal{G}}\left(\mathbb{R}^{3}, B^{+}(2 m, R)\right), B^{+}(2 m, R)=\left\{x \in \mathbb{R}^{3} \mid 2 m \leqslant\|x\| \leqslant R\right\}$.

$$
\begin{gathered}
h^{-1}(r)=\left\{\begin{array}{c}
-\frac{r}{r-2 m}, r>2 m \\
\infty, r=2 m
\end{array}\right\} \longmapsto\left(h_{\epsilon}^{+}\right)^{-1}(r)= \\
h^{-}(r)=\left\{\begin{array}{c}
-\frac{r-2 m}{r} \text { if } r \leqslant 2 m \\
0 \text { if } r \geq 2 m
\end{array}\right\} \longmapsto h_{\epsilon}^{-}(r)= \\
=\left(\frac{\sqrt{(2 m-r)^{2}+\epsilon^{2}}}{r}\right)_{\epsilon} \in \widetilde{\mathcal{G}}\left(\mathbb{R}^{3}, B^{-}(0,2 m)\right), \\
\text { where } B^{-}(0,2 m)=\left\{x \in \mathbb{R}^{3} \mid 0<\|x\| \leqslant 2 m\right\} \\
\quad\left\{\begin{array}{c}
-\frac{r}{r-2 m}, r<2 m \\
\infty, r=2 m
\end{array}\right\} \longmapsto\left(h_{\epsilon}^{-}\right)^{-1}(r)= \\
=\left(\frac{r}{\sqrt{(r-2 m)^{2}+\epsilon^{2}}}\right)_{\epsilon} \in \widetilde{\mathcal{G}}\left(\mathbb{R}^{3}, B^{-}(0,2 m)\right)
\end{gathered}
$$

Inserting (3.2) into (3.1) we obtain a generalized object modeling the singular Schwarzschild metric above (below) gorizon, i.e.,

$$
\left\{\begin{array}{c}
\left(d s_{\epsilon}^{+2}\right)_{\epsilon}=\left(h_{\epsilon}^{+}(r) d t^{2}\right)_{\epsilon}-\left(\left[h_{\epsilon}^{+}(r)\right]^{-1} d r^{2}\right)_{\epsilon}+r^{2} d \Omega^{2} \\
\left(d s_{\epsilon}^{-2}\right)_{\epsilon}=\left(h_{\epsilon}^{-}(r) d t^{2}\right)_{\epsilon}-\left(\left[h_{\epsilon}^{-}(r)\right]^{-1} d r^{2}\right)_{\epsilon}+r^{2} d \Omega^{2}
\end{array}\right.
$$

The generalized Ricci tensor above horizon $\left[\mathbf{R}^{+}\right]_{\alpha}^{\beta}$ may now be calculated componentwise using the classical formulae

$$
\left\{\begin{array}{c}
\left(\left[\mathbf{R}_{\epsilon}^{+}\right]_{0}^{0}\right)_{\epsilon}=\left(\left[\mathbf{R}_{\epsilon}^{+}\right]_{1}^{1}\right)_{\epsilon}=\frac{1}{2}\left(\left(h_{\epsilon}^{+\prime \prime}\right)_{\epsilon}+\frac{2}{r}\left(h_{\epsilon}^{+\prime}\right)_{\epsilon}\right) \\
\left(\left[\mathbf{R}_{\epsilon}^{+}\right]_{2}^{2}\right)_{\epsilon}=\left(\left[\mathbf{R}_{\epsilon}^{+}\right]_{3}^{3}\right)_{\epsilon}=\frac{\left(h_{\epsilon}^{+\prime}\right)_{\epsilon}}{r}+\frac{1+\left(h_{\epsilon}^{+}\right)_{\epsilon}}{r^{2}}
\end{array}\right.
$$

From (3.2) we obtain 


$$
\begin{aligned}
& \begin{array}{c}
h_{\epsilon}^{+\prime}(r)=-\frac{r-2 m}{r\left[(r-2 m)^{2}+\epsilon^{2}\right]^{1 / 2}}+\frac{\left[(r-2 m)^{2}+\epsilon^{2}\right]^{1 / 2}}{r^{2}}, \\
r\left(h_{\epsilon}^{+\prime}\right)+1+\left(h_{\epsilon}^{+}\right)=
\end{array} \\
& r\left\{-\frac{r-2 m}{r\left[(r-2 m)^{2}+\epsilon^{2}\right]^{1 / 2}}+\frac{\left[(r-2 m)^{2}+\epsilon^{2}\right]^{1 / 2}}{r^{2}}\right\}+1-\frac{\sqrt{(r-2 m)^{2}+\epsilon^{2}}}{r}= \\
& -\frac{r-2 m}{\left[(r-2 m)^{2}+\epsilon^{2}\right]^{1 / 2}}+\frac{\left[(r-2 m)^{2}+\epsilon^{2}\right]^{1 / 2}}{r}+1-\frac{\sqrt{(r-2 m)^{2}+\epsilon^{2}}}{r}= \\
& -\frac{r-2 m}{\left[(r-2 m)^{2}+\epsilon^{2}\right]^{1 / 2}}+1 . \\
& h_{\epsilon}^{\prime \prime}(r)=-\left(\frac{r-2 m}{r\left[(r-2 m)^{2}+\epsilon^{2}\right]^{1 / 2}}\right)^{\prime}+\left(\frac{\left[(r-2 m)^{2}+\epsilon^{2}\right]^{1 / 2}}{r^{2}}\right)^{\prime}= \\
& =-\frac{1}{r\left[(r-2 m)^{2}+\epsilon^{2}\right]^{1 / 2}}+\frac{(r-2 m)^{2}}{r\left[(r-2 m)^{2}+\epsilon^{2}\right]^{3 / 2}}+\frac{r-2 m}{r^{2}\left[(r-2 m)^{2}+\epsilon^{2}\right]^{1 / 2}}+ \\
& +\frac{r-2 m}{r^{2}\left[(r-2 m)^{2}+\epsilon^{2}\right]^{1 / 2}}-\frac{2\left[(r-2 m)^{2}+\epsilon^{2}\right]^{1 / 2}}{r^{3}} \text {. } \\
& r^{2}\left\{-\frac{1}{r\left[(r-2 m)^{2}+\epsilon^{2}\right]^{1 / 2}}+\frac{(r-2 m)^{2}}{r\left[(r-2 m)^{2}+\epsilon^{2}\right]^{3 / 2}}+\frac{r-2 m}{r^{2}\left[(r-2 m)^{2}+\epsilon^{2}\right]^{1 / 2}}+\right. \\
& \left.+\frac{r-2 m}{r^{2}\left[(r-2 m)^{2}+\epsilon^{2}\right]^{1 / 2}}-\frac{2\left[(r-2 m)^{2}+\epsilon^{2}\right]^{1 / 2}}{r^{3}}\right\}+ \\
& +2 r\left\{-\frac{r-2 m}{r\left[(r-2 m)^{2}+\epsilon^{2}\right]^{1 / 2}}+\frac{\left[(r-2 m)^{2}+\epsilon^{2}\right]^{1 / 2}}{r^{2}}\right\}= \\
& -\frac{r}{\left[(r-2 m)^{2}+\epsilon^{2}\right]^{1 / 2}}+\frac{r(r-2 m)^{2}}{\left[(r-2 m)^{2}+\epsilon^{2}\right]^{3 / 2}}+\frac{r-2 m}{\left[(r-2 m)^{2}+\epsilon^{2}\right]^{1 / 2}}+ \\
& +\frac{r-2 m}{\left[(r-2 m)^{2}+\epsilon^{2}\right]^{1 / 2}}-\frac{2\left[(r-2 m)^{2}+\epsilon^{2}\right]^{1 / 2}}{r}+ \\
& -\frac{2(r-2 m)}{\left[(r-2 m)^{2}+\epsilon^{2}\right]^{1 / 2}}+\frac{2\left[(r-2 m)^{2}+\epsilon^{2}\right]^{1 / 2}}{r}= \\
& -\frac{r}{\left[(r-2 m)^{2}+\epsilon^{2}\right]^{1 / 2}}+\frac{r(r-2 m)^{2}}{\left[(r-2 m)^{2}+\epsilon^{2}\right]^{3 / 2}} .
\end{aligned}
$$

Investigating the weak limit of the angular components of the Ricci tensor using the abbreviation

$$
\tilde{\Phi}(r)=\int_{0}^{\pi} \sin \theta d \theta \int_{0}^{2 \pi} d \phi \Phi(x)
$$

and let $\Phi(x)$ be the function $\Phi(x) \in \mathcal{S}_{2 m}^{+}\left(\mathbb{R}^{3}\right)$, where by $\mathcal{S}_{2 m}^{+}\left(\mathbb{R}^{3}\right)$ we denote the class of all functions $\Phi(x)$ with compact support such that:

(i) $\operatorname{supp}(\Phi(x)) \subset\{x \mid\|x\| \geq 2 m\} \quad$ (ii) $\tilde{\Phi}(r) \in C^{\infty}(\mathbb{R})$. Then for any function $\Phi(x) \in \mathcal{S}_{2 m}\left(\mathbb{R}^{3}\right)$ we get: 


$$
\begin{gathered}
\int_{K}\left(\left[\mathbf{R}_{\epsilon}^{+}\right]_{2}^{2}\right)_{\epsilon} \Phi(\vec{x}) d^{3} x=\int_{K}\left(\left[\mathbf{R}_{\epsilon}^{+}\right]_{3}^{3}\right)_{\epsilon} \Phi(\vec{x}) d^{3} x= \\
\int_{2 m}^{R}\left(r\left(h_{\epsilon}^{+\prime}\right)_{\epsilon}+1+\left(h_{\epsilon}^{+}\right)_{\epsilon}\right) \tilde{\Phi}(r) d r= \\
\int_{2 m}^{R}\left\{-\frac{r-2 m}{\left[(r-2 m)^{2}+\epsilon^{2}\right]^{1 / 2}}\right\} \tilde{\Phi}(r) d r+\int_{2 m}^{R} \tilde{\Phi}(r) d r .
\end{gathered}
$$

By replacement $\mathrm{r}-2 \mathrm{~m}=\mathrm{u}$, from (3.6) we obtain

$$
\begin{gathered}
\int_{K}\left(\left[\mathbf{R}_{\epsilon}^{+}\right]_{2}^{2}\right){ }_{\epsilon} \Phi(x) d^{3} x= \\
\int_{K}\left(\left[\mathbf{R}_{\epsilon}^{+}\right]_{3}^{3}\right)_{\epsilon} \Phi(x) d^{3} x= \\
-\int_{0}^{R-2 m} \frac{u \tilde{\Phi}(u+2 m) d u}{\left(u^{2}+\epsilon^{2}\right)^{1 / 2}}+\int_{0}^{R-2 m} \tilde{\Phi}(u+2 m) d u .
\end{gathered}
$$

By replacement $\mathrm{u}=\epsilon \eta$, from (3.7) we obtain the expression

$$
\left\{\begin{aligned}
\mathbf{I}_{3}^{+}(\epsilon) & =\int_{K}\left(\left[\mathbf{R}_{\epsilon}^{+}\right]_{3}^{3}\right)_{\epsilon} \Phi(x) d^{3} x=\mathbf{I}_{2}^{+}(\epsilon)=\int_{K}\left(\left[\mathbf{R}_{\epsilon}^{+}\right]_{2}^{2}\right)_{\epsilon} \Phi(\vec{x}) d^{3} x= \\
& -\epsilon \times\left(\int_{0}^{\frac{R-2 m}{\epsilon}} \frac{\eta \tilde{\Phi}(\epsilon \eta+2 m) d \eta}{\left(\eta^{2}+1\right)^{1 / 2}}-\int_{0}^{\frac{R-2 m}{\epsilon}} \tilde{\Phi}(\epsilon \eta+2 m) d \eta\right) .
\end{aligned}\right.
$$

From Eq. (3.8) we obtain

$$
\begin{aligned}
\mathbf{I}_{3}^{+}(\epsilon)= & \mathbf{I}_{2}^{+}(\epsilon)=-\epsilon \frac{\tilde{\Phi}(2 m)}{0 !} \int_{0}^{\frac{R-2 m}{\epsilon}}\left[\frac{\eta}{\left(\eta^{2}+1\right)^{1 / 2}}-1\right] d \eta- \\
& -\frac{\epsilon^{2}}{1 !} \int_{0}^{\frac{R-2 m}{\epsilon}}\left[\frac{\eta}{\left(\eta^{2}+1\right)^{1 / 2}}-1\right] \tilde{\Phi}^{(1)}(\xi) \eta d \eta= \\
-\epsilon \tilde{\Phi}(2 m) & {\left[\sqrt{\left(\frac{R-2 m}{\epsilon}\right)^{2}+1}-\left(\frac{R-2 m}{\epsilon}-1\right)\right]-} \\
& -\frac{\epsilon^{2}}{1} \int_{0}^{\frac{R-2 m}{\epsilon}}\left[\frac{\eta}{\left(\eta^{2}+1\right)^{1 / 2}}-1\right] \tilde{\Phi}^{(1)}(\xi) \eta d \eta,
\end{aligned}
$$

where we have expressed the function $\widetilde{\Phi}(\epsilon \eta+2 m)$ as

$$
\left\{\begin{array}{c}
\widetilde{\Phi}(\epsilon \eta+2 m)=\sum_{l=0}^{n-1} \frac{\Phi^{(l)}(2 m)}{l !}(\epsilon \eta)^{l}+\frac{1}{n !}(\epsilon \eta)^{n} \Phi^{(n)}(\xi), \\
\xi \triangleq \theta \epsilon \eta+2 m, 1>\theta>0, n=1
\end{array}\right.
$$

with $\tilde{\Phi}^{(l)}(\xi) \triangleq d^{l} \tilde{\Phi} / d \xi^{l}$. Equations (3.9)-(3.10 give

$$
\left\{\begin{array}{c}
\lim _{\epsilon \rightarrow 0}\left\{-\epsilon \tilde{\Phi}(2 m)\left[\sqrt{\left(\frac{R-2 m}{\epsilon}\right)^{2}+1}+1-\frac{R-2 m}{\epsilon}\right]\right\}+ \\
+\lim _{\epsilon \rightarrow 0}\left\{-\frac{\epsilon^{2}}{1} \int_{0}^{\frac{R-2 m}{\epsilon}}\left[\frac{\eta}{\left(\eta^{2}+1\right)^{1 / 2}}-1\right] \tilde{\Phi}^{(1)}(\xi) \eta d \eta\right\}=0
\end{array}\right.
$$

Thus in $\mathcal{S}_{2 m}^{\prime}\left(B_{R}^{+}(2 m)\right) \subset \mathcal{S}_{2 m}^{\prime}\left(\mathbb{R}^{3}\right) \subset \mathcal{D}^{\prime}\left(\mathbb{R}^{3}\right)$, where $B^{+}(2 m, R)=\left\{x \in \mathbb{R}^{3} \mid 2 m \leqslant\|x\| \leqslant R\right\}$ from Eq. (3.11) we obtain 


$$
\left\{\begin{array}{l}
w-\lim _{\epsilon \rightarrow 0}\left[\mathbf{R}_{\epsilon}^{+}\right]_{3}^{3}=\lim _{\epsilon \rightarrow 0} \mathbf{I}_{3}^{+}(\epsilon)=0, \\
w-\lim _{\epsilon \rightarrow 0}\left[\mathbf{R}_{\epsilon}^{+}\right]_{2}^{2}=\lim _{\epsilon \rightarrow 0} \mathbf{I}_{2}^{+}(\epsilon)=0 .
\end{array}\right.
$$

For $\left(\left[\mathbf{R}_{\epsilon}^{+}\right]_{1}^{1}\right)_{\epsilon},\left(\left[\mathbf{R}_{\epsilon}^{+}\right]_{0}^{0}\right)_{\epsilon}$ we get:

$$
\left\{\begin{array}{c}
2 \int_{K}\left(\left[\mathbf{R}_{\epsilon}^{+}\right]_{1}^{1}\right)_{\epsilon} \Phi(x) d^{3} x=2 \int_{K}\left(\left[\mathbf{R}_{\epsilon}^{+}\right]_{0}^{0}\right)_{\epsilon} \Phi(x) d^{3} x= \\
=\int_{2 m}^{R}\left\{-\frac{\int_{2 m}^{R}\left(r^{2}\left(h_{\epsilon}^{+\prime \prime}\right)_{\epsilon}+2 r\left(h_{\epsilon}^{+\prime}\right)_{\epsilon}\right) \tilde{\Phi}(r) d r=}{\left[(r-2 m)^{2}+\epsilon^{2}\right]^{1 / 2}}+\frac{r(r-2 m)^{2}}{\left[(r-2 m)^{2}+\epsilon^{2}\right]^{3 / 2}}\right\} \tilde{\Phi}(r) d r .
\end{array}\right.
$$

By replacement $r-2 m=u$, from (3.13) we obtain

$$
\left\{\begin{array}{c}
\mathbf{I}_{1}^{+}(\epsilon)=2 \int_{K}\left(\left[\mathbf{R}_{\epsilon}^{+}\right]_{1}^{1}\right)_{\epsilon} \Phi(x) d^{3} x=I_{2}^{+}(\epsilon)=2 \int_{K}\left(\left[\mathbf{R}_{\epsilon}^{+}\right]_{0}^{0}\right)_{\epsilon} \Phi(x) d^{3} x \\
=\int_{2 m}^{R}\left(r^{2}\left(h_{\epsilon}^{+\prime \prime}\right)_{\epsilon}+2 r\left(h_{\epsilon}^{+\prime}\right)_{\epsilon}\right) \tilde{\Phi}(r) d r= \\
=\int_{0}^{R-2 m}\left\{-\frac{u+2 m}{\left(u^{2}+\epsilon^{2}\right)^{1 / 2}}+\frac{u^{2}(u+2 m)}{\left(u^{2}+\epsilon^{2}\right)^{3 / 2}}\right\} \tilde{\Phi}(u+2 m) d u
\end{array}\right.
$$

By replacement $u=\epsilon \eta$, from (3.14) we obtain

$$
\begin{aligned}
& 2 \int_{K}\left(\left[\mathbf{R}_{\epsilon}^{+}\right]_{1}^{1}\right)_{\epsilon} \Phi(x) d^{3} x=2 \int_{K}\left(\left[\mathbf{R}_{\epsilon}^{+}\right]_{0}^{0}\right)_{\epsilon} \Phi(x) d^{3} x= \\
& =\int_{2 m}^{R}\left(r^{2}\left(h_{\epsilon}^{+\prime \prime}\right)_{\epsilon}+2 r\left(h_{\epsilon}^{+\prime}\right)_{\epsilon}\right) \tilde{\Phi}(r) d r= \\
& =\epsilon \int_{0}^{\frac{R-2 m}{\epsilon}}\left\{-\frac{\epsilon \eta+2 m}{\left(\epsilon^{2} \eta^{2}+\epsilon^{2}\right)^{1 / 2}}+\frac{\epsilon^{2} \eta^{2}(\epsilon \eta+2 m)}{\left(\epsilon^{2} \eta^{2}+\epsilon^{2}\right)^{3 / 2}}\right\} \tilde{\Phi}(\epsilon \eta+2 m) d \eta= \\
& -\int_{0}^{\frac{R-2 m}{\epsilon}} \frac{\epsilon^{2} \eta \tilde{\Phi}(\epsilon \eta+2 m) d \eta}{\left(\epsilon^{2} \eta^{2}+\epsilon^{2}\right)^{1 / 2}}-2 m \int_{0}^{\frac{R-2 m}{\epsilon}} \frac{\epsilon \tilde{\Phi}(\epsilon \eta+2 m) d \eta}{\left(\epsilon^{2} \eta^{2}+\epsilon^{2}\right)^{1 / 2}}+ \\
& \int_{0}^{\frac{R-2 m}{\epsilon}} \frac{\epsilon^{4} \eta^{3} \tilde{\Phi}(\epsilon \eta+2 m) d \eta}{\left(\epsilon^{2} \eta^{2}+\epsilon^{2}\right)^{3 / 2}}+2 m \int_{0}^{\frac{R-2 m}{\epsilon}} \frac{\epsilon^{3} \eta^{2} \tilde{\Phi}(\epsilon \eta+2 m) d \eta}{\left(\epsilon^{2} \eta^{2}+\epsilon^{2}\right)^{3 / 2}}= \\
& \epsilon\left[-\int_{0}^{\frac{R-2 m}{\epsilon}} \frac{\eta \tilde{\Phi}(\epsilon \eta+2 m) d \eta}{\left(\eta^{2}+1\right)^{1 / 2}}+\int_{0}^{\frac{R-2 m}{\epsilon}} \frac{\eta^{3} \tilde{\Phi}(\epsilon \eta+2 m) d \eta}{\left(\eta^{2}+1\right)^{3 / 2}}\right]+ \\
& 2 m\left[-\int_{0}^{\frac{R-2 m}{\epsilon}} \frac{\tilde{\Phi}(\epsilon \eta+2 m) d \eta}{\left(\eta^{2}+1\right)^{1 / 2}}+\int_{0}^{\frac{R-2 m}{\epsilon}} \frac{\eta^{2} \tilde{\Phi}(\epsilon \eta+2 m) d \eta}{\left(\eta^{2}+1\right)^{3 / 2}}\right] .
\end{aligned}
$$

From Eq. (3.15) we obtain

$$
\begin{aligned}
\mathbf{I}_{0}^{+}(\epsilon)= & \mathbf{I}_{1}^{+}(\epsilon)=2 m \frac{\tilde{\Phi}(2 m)}{0 !} \int_{0}^{\frac{R-2 m}{\epsilon}}\left[-\frac{1}{\left(\eta^{2}+1\right)^{1 / 2}}+\frac{\eta^{2}}{\left(\eta^{2}+1\right)^{3 / 2}}\right] d \eta+ \\
& +\frac{\epsilon}{1 !} \int_{0}^{\frac{R-2 m}{\epsilon}} \tilde{\Phi}^{(1)}(\xi)\left[-\frac{1}{\left(\eta^{2}+1\right)^{1 / 2}}+\frac{\eta^{2}}{\left(\eta^{2}+1\right)^{3 / 2}}\right] \eta d \eta+ \\
& +\frac{\epsilon \tilde{\Phi}(2 m)}{0 !} \int_{-2 m}^{\frac{R-2 m}{\epsilon}}\left[-\frac{1}{\left(\eta^{2}+1\right)^{1 / 2}}+\frac{\eta^{2}}{\left(\eta^{2}+1\right)^{3 / 2}}\right] d \eta+ \\
& +\frac{\epsilon^{2}}{1 !} \int_{0}^{\frac{R-2 m}{\epsilon}} \tilde{\Phi}^{(1)}(\xi)\left[-\frac{1}{\left(\eta^{2}+1\right)^{1 / 2}}+\frac{\eta^{2}}{\left(\eta^{2}+1\right)^{3 / 2}}\right] \eta d \eta,
\end{aligned}
$$


we have expressed the function $\widetilde{\Phi}(\epsilon \eta+2 m)$ as

$$
\left\{\begin{aligned}
\widetilde{\Phi}(\epsilon \eta+2 m) & =\sum_{l=0}^{n-1} \frac{\Phi^{\alpha \beta(l)}(2 m)}{l !}(\epsilon \eta)^{l}+\frac{1}{n !}(\epsilon \eta)^{n} \Phi^{\alpha \beta(n)}(\xi) \\
\xi & \triangleq \theta \eta+2 m, 1>\theta>0, \quad n=1
\end{aligned}\right.
$$

with $^{(l)}(\xi) \triangleq d^{l} \tilde{\Phi} / d \xi^{l}$. Equation (3.17) gives

$$
\begin{gathered}
w-\lim _{\epsilon \rightarrow 0} \mathbf{I}_{0}^{+}(\epsilon)=w-\lim _{\epsilon \rightarrow 0} \mathbf{I}_{1}^{+}(\epsilon)= \\
2 m \tilde{\Phi}(2 m) \lim _{\epsilon \rightarrow 0}\left\{\int_{0}^{\frac{R-2 m}{\epsilon}}\left[-\frac{1}{\left(\eta^{2}+1\right)^{1 / 2}}+\frac{\eta^{2}}{\left(\eta^{2}+1\right)^{3 / 2}}\right] d \eta\right\}= \\
2 m \tilde{\Phi}(2 m) \lim _{s \rightarrow \infty}\left[\int_{0}^{s} \frac{\eta^{2} d \eta}{\left(\eta^{2}+1\right)^{3 / 2}}-\int_{0}^{s} \frac{d \eta}{\left(\eta^{2}+1\right)^{1 / 2}}\right]= \\
=-2 m \Phi(2 m) .
\end{gathered}
$$

where use is made of the relation

$$
\lim _{s \rightarrow \infty}\left[\int_{0}^{s} \frac{\eta^{2} d \eta}{\left(\eta^{2}+1\right)^{3 / 2}}-\int_{0}^{s} \frac{d \eta}{\left(u^{2}+1\right)^{1 / 2}}\right]=-1
$$

Thus in $\mathcal{S}_{2 m}^{\prime}\left(B^{+}(2 m, R)\right) \subset \mathcal{S}_{2 m}^{\prime}\left(\mathbb{R}^{3}\right)$ we obtain

$$
w-\lim _{\epsilon \rightarrow 0}\left[\mathbf{R}_{\epsilon}^{+}\right]_{1}^{1}=w-\lim _{\epsilon \rightarrow 0}\left[\mathbf{R}_{\epsilon}^{+}\right]_{0}^{0}=-m \tilde{\Phi}(2 m) .
$$

The supergeneralized Ricci tensor below horizon $\left[\mathbf{R}_{\epsilon}^{-}\right]_{\alpha}^{\beta}=\left[\mathbf{R}_{\epsilon}^{-}\right]_{\alpha}^{\beta}$ may now be calculated componentwise using the classical formulae

$$
\left\{\begin{array}{c}
\left(\left[\mathbf{R}_{\epsilon}^{-}\right]_{0}^{0}\right)_{\epsilon}=\left(\left[\mathbf{R}_{\epsilon}^{-}\right]_{1}^{1}\right)_{\epsilon}=\frac{1}{2}\left(\left(h_{\epsilon}^{-\prime \prime}\right)_{\epsilon}+\frac{2}{r}\left(h_{\epsilon}^{-\prime}\right)_{\epsilon}\right), \\
\left(\left[\mathbf{R}_{\epsilon}^{-}\right]_{2}^{2}\right)_{\epsilon}=\left(\left[\mathbf{R}_{\epsilon}^{-}\right]_{3}^{3}\right)_{\epsilon}=\frac{\left(h_{\epsilon}^{-\prime}\right)_{\epsilon}}{r}+\frac{1+\left(h_{\epsilon}^{-}\right)_{\epsilon}}{r^{2}}
\end{array}\right.
$$

From Eq. (3.21) we obtain

$$
\begin{gathered}
h_{\epsilon}^{-}(r)=-\frac{r-2 m}{r} \longmapsto h_{\epsilon}^{-}(r)=\left(\frac{\sqrt{(2 m-r)^{2}+\epsilon^{2}}}{r}\right)=-h_{\epsilon}^{+}(r), r<2 m . \\
h_{\epsilon}^{-\prime}(r)=-h_{\epsilon}^{+\prime}(r)=\frac{r-2 m}{r\left[(r-2 m)^{2}+\epsilon^{2}\right]^{1 / 2}}-\frac{\left[(r-2 m)^{2}+\epsilon^{2}\right]^{1 / 2}}{r^{2}}, \\
r\left(h_{\epsilon}^{-\prime}\right)_{\epsilon}+1+\left(h_{\epsilon}^{-}\right)_{\epsilon}=-r\left(h_{\epsilon}^{+\prime}\right)_{\epsilon}+1-\left(h_{\epsilon}^{+}\right)_{\epsilon}= \\
\frac{\left.r(r-2 m)^{2}+\epsilon^{2}\right]^{1 / 2}}{[(r-1 .} \\
-\frac{r-2 m}{h_{\epsilon}^{-\prime \prime}(r)=-h_{\epsilon}^{+\prime \prime}(r)=} \\
r^{2}\left[\left(h_{\epsilon}^{-\prime \prime}\right)_{\epsilon}+2 r\left(h_{\epsilon}^{-\prime}\right)_{\epsilon}=-r^{2}\left(h_{\epsilon}^{+\prime \prime}\right)_{\epsilon}-2 r\left(h_{\epsilon}^{+\prime}\right)_{\epsilon}=\right. \\
\frac{r}{\left[(r-2 m)^{2}+\epsilon^{2}\right]^{1 / 2}}-\frac{r(r-2 m)^{2}}{\left[(r-2 m)^{2}+\epsilon^{2}\right]^{3 / 2}} .
\end{gathered}
$$


Investigating the weak limit of the angular components of the Ricci tensor (using the abbreviation $\tilde{\Phi}(r)={ }_{0}^{\pi} \sin \theta d \theta \int_{0}^{2 \pi} d \phi \Phi(x)$ where $\Phi(\vec{x}) \in C^{\infty}\left(\mathbb{R}^{3}\right), \Phi(x)$ is a function with compact support $K$ such that $K \subseteq B^{-}(0,2 m)=\left\{x \in \mathbb{R}^{3} \mid 0 \leqslant\|x\| \leqslant 2 m\right\} \quad$ we get:

$$
\begin{gathered}
\int_{K}\left(\left[\mathbf{R}_{\epsilon}^{-}\right]_{2}^{2}\right)_{\epsilon} \Phi(\vec{x}) d^{3} x=\int_{K}\left(\left[\mathbf{R}_{\epsilon}^{-}\right]_{3}^{3}\right)_{\epsilon} \Phi(\vec{x}) d^{3} x= \\
\int_{0}^{2 m}\left(r\left(h_{\epsilon}^{-\prime}\right)_{\epsilon}+1+\left(h_{\epsilon}^{-}\right)_{\epsilon}\right) \tilde{\Phi}(r) d r= \\
\int_{0}^{2 m}\left\{\frac{r-2 m}{\left[(r-2 m)^{2}+\epsilon^{2}\right]^{1 / 2}}\right\} \tilde{\Phi}(r) d r+\int_{0}^{2 m} \tilde{\Phi}(r) d r .
\end{gathered}
$$

By replacement $r-2 m=u$, from Eq. (3.23) we obtain

$$
\left\{\begin{array}{c}
\int_{K}\left(\left[\mathbf{R}_{\epsilon}^{-}\right]_{2}^{2}\right)_{\epsilon} \Phi(x) d^{3} x=\int_{K}\left(\left[\mathbf{R}_{\epsilon}^{-}\right]_{3}^{3}\right)_{\epsilon} \Phi(x) d^{3} x= \\
\int_{-2 m}^{0} \frac{u \Phi(u+2 m) d u}{\left(u^{2}+\epsilon^{2}\right)^{1 / 2}}+\int_{-2 m}^{0} \tilde{\Phi}(u+2 m) d u .
\end{array}\right.
$$

By replacement $\mathrm{u}=\epsilon \eta$, from (3.23) we obtain

$$
\begin{aligned}
\mathbf{I}_{3}^{-}(\epsilon)= & \int_{K}\left(\left[\mathbf{R}_{\epsilon}^{-}\right]_{3}^{3}\right)_{\epsilon} \Phi(x) d^{3} x=\mathbf{I}_{2}^{-}(\epsilon)=\int_{K}\left(\left[\mathbf{R}_{\epsilon}^{-}\right]_{2}^{2}\right)_{\epsilon} \Phi(\vec{x}) d^{3} x= \\
& \epsilon \times\left(\int_{-\frac{2 m}{\epsilon}}^{0} \frac{\eta \tilde{\Phi}(\epsilon \eta+2 m) d \eta}{\left(\eta^{2}+1\right)^{1 / 2}}+\int_{-\frac{2 m}{\epsilon}}^{0} \tilde{\Phi}(\epsilon \eta+2 m) d \eta\right)
\end{aligned}
$$

which is calculated to give

$$
\begin{aligned}
\mathbf{I}_{3}^{-}(\epsilon) & =\mathbf{I}_{2}^{-}(\epsilon)=\epsilon \frac{\tilde{\Phi}(2 m)}{0 !} \int_{-\frac{2 m}{\epsilon}}^{0}\left[\frac{\eta}{\left(\eta^{2}+1\right)^{1 / 2}}+1\right] d \eta+ \\
& +\frac{\epsilon^{2}}{1 !} \int_{-\frac{2 m}{\epsilon}}^{0}\left[\frac{\eta}{\left(\eta^{2}+1\right)^{1 / 2}}+1\right] \tilde{\Phi}^{(1)}(\xi) \eta d \eta= \\
& \epsilon \tilde{\Phi}(2 m)\left[1-\sqrt{\left(\frac{2 m}{\epsilon}\right)^{2}+1}+\frac{2 m}{\epsilon}\right]+ \\
& +\frac{\epsilon^{2}}{1} \int_{-\frac{2 m}{\epsilon}}^{0}\left[\frac{\eta}{\left(\eta^{2}+1\right)^{1 / 2}}+1\right] \tilde{\Phi}^{(1)}(\xi) \eta d \eta,
\end{aligned}
$$

where we have expressed the function $\widetilde{\Phi}(\epsilon \eta+2 m)$ as

$$
\begin{gathered}
\left\{\begin{array}{c}
\widetilde{\Phi}(\epsilon \eta+2 m)=\sum_{l=0}^{n-1} \frac{\Phi^{(l)}(2 m)}{l !}(\epsilon \eta)^{l}+\frac{1}{n !}(\epsilon \eta)^{n} \Phi^{(n)}(\xi), \\
\xi \triangleq \theta \epsilon \eta+2 m, 1>\theta>0, \quad n=1
\end{array}\right. \\
\text { with } \tilde{\Phi}^{(l) \triangleq d^{l} \tilde{\Phi} / d r^{l} \text {. Equation (3.27) gives }}
\end{gathered}
$$

with $\tilde{\Phi}^{(l)} \triangleq d^{l} \tilde{\Phi} / d r^{l}$. Equation (3.27) gives 


$$
\left\{\begin{array}{c}
\lim _{\epsilon \rightarrow 0} \mathbf{I}_{3}^{-}(\epsilon)=\lim _{\epsilon \rightarrow 0} \mathbf{I}_{2}^{-}(\epsilon)= \\
\lim _{\epsilon \rightarrow 0}\left\{\epsilon \tilde{\Phi}(2 m)\left[1-\sqrt{\left(\frac{2 m}{\epsilon}\right)^{2}+1}+\frac{2 m}{\epsilon}\right]\right\}+ \\
+\lim _{\epsilon \rightarrow 0}\left\{\frac{\epsilon^{2}}{2} \int_{-\frac{2 m}{\epsilon}}^{0}\left[\frac{\eta}{\left(\eta^{2}+1\right)^{1 / 2}}+1\right] \tilde{\Phi}^{(1)}(\xi) \eta d \eta\right\}=0 .
\end{array}\right.
$$

Thus in $\mathcal{S}_{2 m}^{\prime}\left(B_{R}^{-}(2 m)\right) \subset \mathcal{S}_{2 m}^{\prime}\left(\mathbb{R}^{3}\right)$, where $B^{-}(0,2 m)=\left\{x \in \mathbb{R}^{3} \mid 0 \leqslant\|x\| \leqslant 2 m\right\}$ from Eq. (3.28) we obtain

$$
\left\{\begin{array}{l}
w-\lim _{\epsilon \rightarrow 0}\left[\mathbf{R}_{\epsilon}^{-}\right]_{3}^{3}=\lim _{\epsilon \rightarrow 0} \mathbf{I}_{3}^{-}(\epsilon)=0 . \\
w-\lim _{\epsilon \rightarrow 0}\left[\mathbf{R}_{\epsilon}^{-}\right]_{2}^{2}=\lim _{\epsilon \rightarrow 0} \mathbf{I}_{2}^{-}(\epsilon)=0 .
\end{array}\right.
$$

For $\left(\left[\mathbf{R}_{\epsilon}^{-}\right]_{1}^{1}\right)_{\epsilon},\left(\left[\mathbf{R}_{\epsilon}^{-}\right]_{0}^{0}\right)_{\epsilon}$ we get:

$$
\begin{aligned}
& 2 \int_{K}\left(\left[\mathbf{R}_{\epsilon}^{-}\right]_{1}^{1}\right)_{\epsilon} \Phi(x) d^{3} x=2 \int_{K}\left(\left[\mathbf{R}_{\epsilon}^{-}\right]_{0}^{0}\right)_{\epsilon} \Phi(x) d^{3} x= \\
= & \int_{0}^{2 m}\left\{\frac{\int_{0}^{2 m}\left(r^{2}\left(h_{\epsilon}^{-\prime \prime}\right)_{\epsilon}+2 r\left(h_{\epsilon}^{-\prime}\right)_{\epsilon}\right) \tilde{\Phi}(r) d r=}{\left[(r-2 m)^{2}+\epsilon^{2}\right]^{1 / 2}}-\frac{r(r-2 m)^{2}}{\left[(r-2 m)^{2}+\epsilon^{2}\right]^{3 / 2}}\right\} \tilde{\Phi}(r) d r .
\end{aligned}
$$

By replacement $r-2 m=u$, from (3.30) we obtain

$$
\begin{gathered}
I_{1}^{+}(\epsilon)=2 \int\left(\left[\mathbf{R}_{\epsilon}^{-}\right]_{1}^{1}\right)_{\epsilon} \Phi(x) d^{3} x=I_{2}^{+}(\epsilon)=2 \int\left(\left[\mathbf{R}_{\epsilon}^{-}\right]_{0}^{0}\right)_{\epsilon} \Phi(x) d^{3} x \\
=\int_{0}^{2 m}\left(r^{2}\left(h_{\epsilon}^{-\prime \prime}\right)_{\epsilon}+2 r\left(h_{\epsilon}^{-\prime}\right)_{\epsilon}\right) \tilde{\Phi}(r) d r= \\
=\int_{-2 m}^{0}\left\{\frac{u+2 m}{\left(u^{2}+\epsilon^{2}\right)^{1 / 2}}-\frac{u^{2}(u+2 m)}{\left(u^{2}+\epsilon^{2}\right)^{3 / 2}}\right\} \tilde{\Phi}(u+2 m) d u .
\end{gathered}
$$

By replacement $u=\epsilon \eta$, from (3.31) we obtain

$$
\begin{aligned}
& 2 \int_{K}\left(\left[\mathbf{R}_{\epsilon}^{-}\right]_{1}^{1}\right)_{\epsilon} \Phi(x) d^{3} x=2 \int_{K}\left(\left[\mathbf{R}_{\epsilon}^{-}\right]_{0}^{0}\right)_{\epsilon} \Phi(x) d^{3} x= \\
& \int_{-\frac{2 m}{\epsilon}}^{0}\left(r^{2}\left(h_{\epsilon}^{-\prime \prime}\right)_{\epsilon}+2 r\left(h_{\epsilon}^{-\prime}\right)_{\epsilon}\right) \tilde{\Phi}(r) d r= \\
& =\epsilon \int_{-\frac{2 m}{\epsilon}}^{0}\left\{\frac{\epsilon \eta+2 m}{\left(\epsilon^{2} \eta^{2}+\epsilon^{2}\right)^{1 / 2}}-\frac{\epsilon^{2} \eta^{2}(\epsilon \eta+2 m)}{\left(\epsilon^{2} \eta^{2}+\epsilon^{2}\right)^{3 / 2}}\right\} \tilde{\Phi}(\epsilon \eta+2 m) d \eta= \\
& \int_{-\frac{2 m}{\epsilon}}^{0} \frac{\epsilon^{2} \eta \tilde{\Phi}(\epsilon \eta+2 m) d \eta}{\left(\epsilon^{2} \eta^{2}+\epsilon^{2}\right)^{1 / 2}}+2 m \int_{-\frac{2 m}{\epsilon}}^{0} \frac{\epsilon \tilde{\Phi}(\epsilon \eta+2 m) d \eta}{\left(\epsilon^{2} \eta^{2}+\epsilon^{2}\right)^{1 / 2}}- \\
& -\int_{-\frac{2 m}{\epsilon}}^{0} \frac{\epsilon^{4} \eta^{3} \tilde{\Phi}(\epsilon \eta+2 m) d \eta}{\left(\epsilon^{2} \eta^{2}+\epsilon^{2}\right)^{3 / 2}}-2 m \int_{-\frac{2 m}{\epsilon}}^{0} \frac{\epsilon^{3} \eta^{2} \tilde{\Phi}(\epsilon \eta+2 m) d \eta}{\left(\epsilon^{2} \eta^{2}+\epsilon^{2}\right)^{3 / 2}}= \\
& \epsilon \int_{-\frac{2 m}{\epsilon}}^{0} \frac{\eta \tilde{\Phi}(\epsilon \eta+2 m) d \eta}{\left(\eta^{2}+1\right)^{1 / 2}}-\int_{-\frac{2 m}{\epsilon}}^{0} \frac{\eta^{3} \tilde{\Phi}(\epsilon \eta+2 m) d \eta}{\left(\eta^{2}+1\right)^{3 / 2}}+ \\
& +2 m\left[\int_{-\frac{2 m}{\epsilon}}^{0} \frac{\tilde{\Phi}(\epsilon \eta+2 m) d \eta}{\left(\eta^{2}+1\right)^{1 / 2}}-\int_{-\frac{2 m}{\epsilon}}^{0} \frac{\eta^{2} \tilde{\Phi}(\epsilon \eta+2 m) d \eta}{\left(\eta^{2}+1\right)^{3 / 2}}\right] .
\end{aligned}
$$


which is calculated to give

$$
\begin{gathered}
\mathbf{I}_{0}^{-}(\epsilon)=\mathbf{I}_{1}^{-}(\epsilon)=2 m \frac{\tilde{\Phi}(2 m)}{0 !} \epsilon^{l} \int_{-\frac{2 m}{\epsilon}}^{0}\left[\frac{1}{\left(\eta^{2}+1\right)^{1 / 2}}-\frac{\eta^{2}}{\left(\eta^{2}+1\right)^{3 / 2}}\right] d \eta+ \\
+\frac{\epsilon}{1 !} \int_{0}^{\frac{2 m}{\epsilon}} \tilde{\Phi}^{(1)}(\xi)\left[\frac{1}{\left(\eta^{2}+1\right)^{1 / 2}}-\frac{\eta^{2}}{\left(\eta^{2}+1\right)^{3 / 2}}\right] \eta d \eta+O\left(\epsilon^{2}\right) .
\end{gathered}
$$

where we have expressed the function $\widetilde{\Phi}(\epsilon \eta+2 m)$ as

$$
\begin{aligned}
\widetilde{\Phi}(\epsilon \eta+2 m) & =\sum_{l=0}^{n-1} \frac{\Phi^{\alpha \beta(l)}(2 m)}{l !}(\epsilon \eta)^{l}+\frac{1}{n !}(\epsilon \eta)^{n} \Phi^{\alpha \beta(n)}(\xi), \\
\xi & \triangleq \theta \epsilon \eta+2 m, 1>\theta>0, \quad n=1
\end{aligned}
$$

with $^{(l)}(\xi) \triangleq d^{l} \tilde{\Phi} / d \xi^{l}$. Equation (3.34) gives

$$
\begin{gathered}
\lim _{\epsilon \rightarrow 0} \mathbf{I}_{0}^{-}(\epsilon)=\lim _{\epsilon \rightarrow 0} \mathbf{I}_{1}^{-}(\epsilon)= \\
2 m \lim _{\epsilon \rightarrow 0}\left\{\frac{\tilde{\Phi}(2 m)}{0 !} \int_{-\frac{2 m}{\epsilon}}^{0}\left[\frac{1}{\left(\eta^{2}+1\right)^{1 / 2}}-\frac{\eta^{2}}{\left(\eta^{2}+1\right)^{3 / 2}}\right] d \eta\right\}= \\
2 m \tilde{\Phi}(2 m) \lim _{s \rightarrow 0}\left[\int_{-s}^{0} \frac{d \eta}{\left(\eta^{2}+1\right)^{1 / 2}}-\int_{-s}^{0} \frac{\eta^{2} d \eta}{\left(\eta^{2}+1\right)^{3 / 2}}\right]= \\
=2 m \tilde{\Phi}(2 m) .
\end{gathered}
$$

where use is made of the relation

$$
\lim _{s \rightarrow \infty}\left[\int_{-s}^{0} \frac{d \eta}{\left(u^{2}+1\right)^{1 / 2}}-\int_{-s}^{0} \frac{\eta^{2} d \eta}{\left(\eta^{2}+1\right)^{3 / 2}}\right]=1 .
$$

Thus in $\mathcal{S}^{\prime}\left(B^{-}(0,2 m)\right) \subset \mathcal{S}^{\prime}\left(\mathbb{R}^{3}\right)$ we obtain $w-\lim _{\epsilon \rightarrow 0}\left[\mathbf{R}_{\epsilon}^{-}\right]_{1}^{1}=w-\lim _{\epsilon \rightarrow 0}\left[\mathbf{R}_{\epsilon}^{-}\right]_{0}^{0}=m \tilde{\Phi}(2 m)$.

Using Eqs. (3.12), (3.20), (3.29), (3.37) we obtain

$$
\int\left[\left(\mathbf{T}_{r}^{+r}+\mathbf{T}_{\theta}^{+\theta}+\mathbf{T}_{\phi}^{+\phi}+\mathbf{T}_{t}^{+t}\right)+\left(\mathbf{T}_{r}^{-r}+\mathbf{T}_{\theta}^{-\theta}+\mathbf{T}_{\phi}^{-\phi}+\mathbf{T}_{t}^{-t}\right)\right] \sqrt{-g} d^{3} x=0
$$

Thus the Tolman formula [8], [9] for the total energy of a static and asymptotically flat spacetime with $g$ the determinant of the four-dimensional metric and $d^{3} x$ the coordinate volume element, gives

$$
E_{T}=\int\left(\mathbf{T}_{r}^{r}+\mathbf{T}_{\theta}^{\theta}+\mathbf{T}_{\phi}^{\phi}+\mathbf{T}_{t}^{t}\right) \sqrt{-g} d^{3} x=m
$$

We rewrite now the Schwarzschild metric (3.3) in the form

$$
\left\{\begin{array}{c}
\left(d s_{\epsilon}^{ \pm 2}\right)_{\epsilon}=\left(h_{\epsilon}^{ \pm}(r) d t^{2}\right)_{\epsilon}-\left(\left(1+C_{\epsilon}^{ \pm}(r)\right) d r^{2}\right)_{\epsilon}+r^{2} d \Omega^{2} \\
C_{\epsilon}^{ \pm}(r)=-1+\left[h_{\epsilon}^{ \pm}(r)\right]^{-1} .
\end{array}\right.
$$

Using Eq. (A.5) from Eq. (3.40) one obtains for $r \asymp 2 m$

$$
\begin{gathered}
\left(\mathbf{R}^{ \pm \mu \nu}(\epsilon) \mathbf{R}_{\mu \nu}^{ \pm}(\epsilon)\right)_{\epsilon}= \\
\left(\left(\frac{1}{2}\left(h_{\epsilon}^{ \pm}\right)^{\prime \prime}+\frac{1}{r}\left(h_{\epsilon}^{ \pm}\right)^{\prime}\right)^{2}\right)_{\epsilon}+2\left(\left[-\frac{\left(h_{\epsilon}^{ \pm}\right)^{\prime}}{r}+\frac{1}{r^{2}}\right]^{2}\right)_{\epsilon}+ \\
+\left(\left[\frac{1}{2}\left(h_{\epsilon}^{ \pm}\right)^{\prime \prime}+\frac{\left(h_{\epsilon}^{ \pm}\right)^{\prime}}{r}\right]^{2}\right)_{\epsilon} \asymp \frac{1}{4\left[(r-2 m)^{2}+\epsilon^{2}\right]}+\frac{2}{(2 m)^{4}},
\end{gathered}
$$


and

$$
\begin{gathered}
\left(\mathbf{R}^{\rho \sigma \mu \nu}(\epsilon) \mathbf{R}_{\rho \sigma \mu \nu}(\epsilon)\right)_{\epsilon}= \\
\left(\left(\left(h_{\epsilon}^{ \pm}\right)^{\prime \prime}\right)^{2}+2\left(\frac{\left(h_{\epsilon}^{ \pm}\right)^{\prime}}{r}\right)^{2}+4 \frac{1}{r^{4}}+2\left(\frac{\left(h_{\epsilon}^{ \pm}\right)^{\prime}}{r}\right)^{2}\right)_{\epsilon} \asymp \\
\asymp \frac{1}{\left[(r-2 m)^{2}+\epsilon^{2}\right]}+\frac{4}{(2 m)^{4}} .
\end{gathered}
$$

\subsection{Examples of distributional geometries calculation of the distribu- tional quadratic scalars by using nonsmooth regularization via horizon}

Let us consider again the Schwarzschild metric (3.1)

$$
\left\{\begin{array}{c}
d s^{2}=h(r) d t^{2}-h(r)^{-1} d r^{2}+r^{2} d \Omega^{2} \\
h(r)=-1+\frac{2 m}{r}=-\frac{r-2 m}{r} \\
h^{-1}(r)=-\frac{r}{r-2 m}
\end{array}\right.
$$

We rewrite now the Schwarzschild metric (3.43) above Horizon $(r \geq 2 m)$ in the form

$$
\left\{\begin{array}{c}
d s^{+2}=-A^{+}(r) d t^{2}+\left(A^{+}(r)\right)^{-1}(r) d r^{2}+r^{2} d \Omega^{2}, \\
A^{+}(r)=\frac{r-2 m}{r}, \\
\left(A^{+}(r)\right)^{-1}=\frac{r}{r-2 m} .
\end{array}\right.
$$

Following the above discussion we consider the singular metric coefficient $A^{-1}(r)$ as an element of $\mathrm{D}^{\prime}\left(\mathbb{R}^{3}\right)$ and embed it into $\left(\mathcal{G}\left(\mathbb{R}^{3}\right)\right)$ by replacement

$$
r-2 m \longmapsto \sqrt{r^{2}+\epsilon^{2}}-2 m \text {. }
$$

Thus above Horizon $(r \geq 2 m)$ the corresponding distributional metric $\widetilde{d} s_{\epsilon \epsilon}^{+2}$ takes the form

$$
\left\{\begin{array}{c}
\left(\widetilde{d} s_{\epsilon}^{+2}\right)_{\epsilon}=\left(-A_{\epsilon}^{+}(r) d t^{2}+\left(A_{\epsilon}^{+}(r)\right)^{-1} d r^{2}\right)_{\epsilon}+r^{2} d \Omega^{2}, \\
\left(A_{\epsilon}^{+}(r)\right)_{\epsilon}=\left(\frac{\sqrt{r^{2}+\epsilon^{2}}-2 m}{r}\right)_{\epsilon} \\
\left(\left(A_{\epsilon}^{+}(r)\right)^{-1}\right)_{\epsilon}=\left(\frac{r}{\sqrt{r^{2}+\epsilon^{2}}-2 m}\right)_{\epsilon} .
\end{array}\right.
$$

We revrite now the Schwarzschild metric (3.43) below Horizon $(r<2 m)$ in the form

$$
\left\{\begin{array}{c}
d s^{-2}=A^{-}(r) d t^{2}-\left(A^{-}(r)\right)^{-1} d r^{2}+r^{2} d \Omega^{2}, \\
A^{-}(r)=\frac{2 m-r}{r},\left(A^{-}(r)\right)^{-1}=\frac{r}{2 m-r} .
\end{array}\right.
$$

Following the above discussion we consider the singular metric coefficient $A^{-1}(r)$ as an element of $\mathcal{D}^{\prime}\left(\mathbb{R}^{3}\right)$ and embed it into $\left(\mathcal{G}\left(\mathbb{R}^{3}\right)\right)$ by replacement

$$
2 m-r \longmapsto 2 m-\sqrt{r^{2}+\epsilon^{2}} .
$$

Thus below Horizon $(r<2 m)$ the corresponding distributional metric $\left(\widetilde{d} s_{\epsilon}^{-2}\right)_{\epsilon}$ takes the form 


$$
\left\{\begin{array}{c}
\left(d \widetilde{s}_{\epsilon}^{-2}\right)_{\epsilon}=\left(A_{\epsilon}^{-}(r) d t^{2}-\left(A_{\epsilon}^{-}(r)\right)^{-1} d r^{2}\right)_{\epsilon}+r^{2} d \Omega^{2}, \\
\left(A_{\epsilon}^{-}(r)\right)_{\epsilon}=\left(\frac{2 m-\sqrt{r^{2}+\epsilon^{2}}}{r}\right)_{\epsilon},\left(\left(A_{\epsilon}^{-}(r)\right)^{-1}\right)_{\epsilon}=\left(\frac{r}{2 m-\sqrt{r^{2}+\epsilon^{2}}}\right)_{\epsilon} .
\end{array}\right.
$$

From Eq. (3.46) one obtains

$$
\left\{\begin{array}{c}
\left(A_{\epsilon}^{+}\right)^{\prime}=\left(-\frac{\sqrt{r^{2}+\epsilon^{2}}-2 m}{r}\right)^{\prime}=-\frac{1}{\sqrt{r^{2}+\epsilon^{2}}}+\frac{\sqrt{r^{2}+\epsilon^{2}}-2 m}{r^{2}} \\
\left(A_{\epsilon}^{+}\right)^{\prime \prime}=\frac{r}{\left(r^{2}+\epsilon^{2}\right)^{3 / 2}}-2 \frac{\sqrt{r^{2}+\epsilon^{2}}-2 m}{r^{3}}+\frac{1}{r \sqrt{r^{2}+\epsilon^{2}}}
\end{array}\right.
$$

From Eq. (3.46) using Eq. (A.5) one obtains

$$
\begin{gathered}
(\mathbf{R}(\epsilon))_{\epsilon}=\left(-\frac{4 A_{\epsilon}^{\prime}}{r}+\frac{2 A_{\epsilon} C_{\epsilon}}{r^{2}}-A_{\epsilon}^{\prime \prime}\right)_{\epsilon}= \\
-\frac{4}{r}\left(-\frac{1}{\sqrt{r^{2}+\epsilon^{2}}}+\frac{\sqrt{r^{2}+\epsilon^{2}}-2 m}{r^{2}}\right)_{\epsilon}+\frac{2}{r^{2}}- \\
-\left(\frac{r}{\left(r^{2}+\epsilon^{2}\right)^{3 / 2}}-2 \frac{\sqrt{r^{2}+\epsilon^{2}}-2 m}{r^{3}}+\frac{1}{r \sqrt{r^{2}+\epsilon^{2}}}\right)_{\epsilon} .
\end{gathered}
$$

From Eq. (3.51) for $r=2 m$ one obtains

$$
(\mathbf{R}(\epsilon))_{\epsilon}=\frac{6}{(2 m)^{2}}-\frac{1}{(2 m)^{2}}+\frac{1}{(2 m)^{3}}=\frac{1}{(2 m)^{3}}+\frac{5}{(2 m)^{2}}
$$

Remark 3.3 Note that curvature scalar $(\mathbf{R}(\epsilon))_{\epsilon}$ again nonzero but nonsingular. Let us introduce now the general metric which has the form:

$$
\left\{\begin{array}{c}
d s^{2}=-A(r)\left(d x^{0}\right)^{2}-2 D(r) d x^{0} d r+\left(B(r)+C(r)(d r)^{2}\right. \\
+B(r) r^{2}\left[(d \theta)^{2}+\sin ^{2} \theta(d \phi)^{2}\right]
\end{array}\right.
$$

where

$$
\left\{\begin{array}{c}
A(r)=\Omega^{2}\left(1-\frac{a}{K(r)}\right), \quad B(r)=\frac{K^{2}(r)}{\rho^{2}(r)} \\
C(r)=\left(1-\frac{a}{K(r)}\right)^{-1}\left(K^{\prime}(r)\right)^{2}-\frac{K^{2}(r)}{\rho^{2}(r)}-\left(1-\frac{a}{K(r)}\right)\left(f^{\prime}\right)^{2} \\
D(r)=\Omega\left(1-\frac{a}{K(r)}\right) f^{\prime}, K^{\prime}(r) \triangleq d K(r) / d r, f^{\prime}(r) \triangleq d f(r) / d r \\
K(r)=\rho(r)-|a|, \\
a<0 .
\end{array}\right.
$$

Remark 3.4 Note that the coordinates $t=x^{0} /$ and $r$ are time and space coordinates, respectively, only if

$$
1-\frac{a}{K}>0, \quad\left(1-\frac{a}{K}\right)^{-1}\left(K^{\prime}\right)^{2}-\left(1-\frac{a}{K}\right)\left(f^{\prime}\right)^{2}>0 .
$$

In the Cartesian coordinate system $\left\{x^{\mu} ; \mu=0,1,2,3\right\}$ with

$$
x^{1}=r \cos \phi \sin \theta, x^{2}=r \sin \phi \sin \theta, x^{3}=r \cos \theta,
$$

the metric (3.53)-(3.55) takes the form 


$$
d s^{2}=g_{\mu \nu} d x^{\mu} d x^{\nu}
$$

with $g_{\mu \nu}$ given by

$$
g_{00}=-A, g_{0 \alpha}=-D \frac{x^{\alpha}}{r}, g_{\alpha \beta}=B \delta^{\alpha \beta}+C \frac{x^{\alpha} x^{\beta}}{r^{2}} .
$$

From Eq. (3.54) one obtains

$$
\begin{gathered}
A(r)=\Omega^{2}\left(\frac{\rho(r)}{\rho(r)-|a|}\right), \quad B=\frac{(\rho(r)-|a|)^{2}}{\rho^{2}(r)}, \\
C(r)=\left(\frac{\rho(r)-|a|}{\rho(r)}\right)-\frac{(\rho(r)-|a|)^{2}}{\rho^{2}(r)}-\left(\frac{\rho(r)}{\rho(r)-|a|}\right)\left(f^{\prime}(r)\right)^{2}, \\
D(r)=\Omega\left(\frac{\rho(r)}{\rho(r)-|a|}\right) f^{\prime}(r), f^{\prime}(r) \triangleq d f(r) / d r .
\end{gathered}
$$

Regularizing the function $(\rho(r)-|a|)^{-1}$ above horizon (under condition $\rho(r)-|a| \geq 0 C$ ) such as

$$
\begin{gathered}
\rho(r)-|a| \geq 0: \\
(\rho(r)-|a|)^{-1} \mapsto\left(\rho_{\epsilon}(r)-|a|\right)^{-1}=\left(\sqrt{\rho^{2}(r)+\epsilon^{2}}-|a|\right)^{-1}
\end{gathered}
$$

with $\epsilon \in(0,1]$ from Eq. (3.59)-Eq. (3.60) one obtains

$$
\begin{gathered}
A_{\epsilon}^{+}(r)=\Omega^{2}\left(\frac{\rho_{\epsilon}(r)}{\rho_{\epsilon}(r)-|a|}\right), \quad B_{\epsilon}^{+}(r)=\frac{\left(\rho_{\epsilon}(r)-|a|\right)^{2}}{\rho_{\epsilon}^{2}(r)}, \\
C_{\epsilon}^{+}(r)=\left(\frac{\rho_{\epsilon}(r)-|a|}{\rho_{\epsilon}(r)}\right)-\frac{\left(\rho_{\epsilon}(r)-|a|\right)^{2}}{\rho_{\epsilon}^{2}(r)}-\left(\frac{\rho_{\epsilon}(r)}{\rho_{\epsilon}(r)-|a|}\right)\left(f^{\prime}(r)\right)^{2}, \\
D_{\epsilon}^{+}(r)=\Omega\left(\frac{\rho_{\epsilon}(r)}{\rho_{\epsilon}(r)-|a|}\right) f^{\prime}(r), f^{\prime}(r) \triangleq d f(r) / d r .
\end{gathered}
$$

Regularizing the function $(|a|-\rho(r))^{-1}$ below horizon (under condition $|a|-\rho(r) \geq 0$ ) such as

$$
\begin{aligned}
& |a|-\rho(r) \geq 0: \\
(|a|-\rho(r))^{-1} \mapsto & \left(|a|-\rho_{\epsilon}(r)\right)=\left(|a|-\sqrt{r^{2}+\epsilon^{2}}\right)^{-1}
\end{aligned}
$$

with $\epsilon \in(0,1]$ from Eq. (3.59)-Eq. (3.62) one obtains

$$
\begin{gathered}
A_{\epsilon}^{-}(r)=-\Omega^{2}\left(\frac{\rho_{\epsilon}(r)}{|a|-\rho_{\epsilon}(r)}\right), \quad B_{\epsilon}^{-}(r)=\frac{\left(|a|-\rho_{\epsilon}(r)\right)^{2}}{\rho_{\epsilon}^{2}(r)}, \\
C_{\epsilon}^{-}(r)=-\left(\frac{|a|-\rho_{\epsilon}(r)}{\rho_{\epsilon}(r)}\right)-\frac{\left(|a|-\rho_{\epsilon}(r)\right)^{2}}{\rho_{\epsilon}^{2}(r)}+\left(\frac{\rho_{\epsilon}(r)}{|a|-\rho_{\epsilon}(r)}\right)\left(f^{\prime}(r)\right)^{2}, \\
D_{\epsilon}^{-}(r)=-\Omega\left(\frac{\rho_{\epsilon}(r)}{|a|-\rho_{\epsilon}(r)}\right) f^{\prime}(r), f^{\prime}(r) \triangleq d f(r) / d r .
\end{gathered}
$$

Remark 3.5 Finally the metric (3.57) becomes the Colombeau object of the form

$$
\left(d s_{\epsilon}^{2}\right)_{\epsilon}=\left(g_{\mu \nu}^{ \pm}(\epsilon) d x^{\mu} d x^{\nu}\right)_{\epsilon}
$$

with $g_{\mu \nu}(\epsilon)$ given by

$$
\begin{gathered}
g_{00}^{ \pm}(\epsilon)=-A_{\epsilon}^{ \pm}(r), \quad g_{0 \alpha}^{ \pm}(\epsilon)=-D_{\epsilon}^{ \pm}(r) \frac{x^{\alpha}}{r} \\
g_{\alpha \beta}^{ \pm}(\epsilon)=B_{\epsilon}^{ \pm}(r) \delta^{\alpha \beta}+C_{\epsilon}^{ \pm}(r) \frac{x^{\alpha} x^{\beta}}{r^{2}}
\end{gathered}
$$


Using now Eq. A2 one obtains that the Colombeau curvature scalars $\left(\mathbf{R}^{ \pm}(\epsilon)\right)_{\epsilon}$ in terms of Colombeau generalized functions $\left(A_{\epsilon}^{ \pm}(r)\right)_{\epsilon},\left(B_{\epsilon}^{ \pm}(r)\right)_{\epsilon},\left(C_{\epsilon}^{ \pm}(r)\right)_{\epsilon},\left(D_{\epsilon}^{ \pm}(r)\right)_{\epsilon}$ is expressed as

$$
\begin{aligned}
\left(\mathbf{R}^{+}(\epsilon)\right)_{\epsilon} & =\left(\frac{r^{2}+\epsilon^{2}}{\left(\sqrt{r^{2}+\epsilon^{2}}-|a|\right)^{2}}\left[\frac{9 a \epsilon^{2}}{\left(r^{2}+\epsilon^{2}\right)^{\frac{5}{2}}}-\frac{2 a \epsilon^{2}}{r^{2}\left(r^{2}+\epsilon^{2}\right)^{\frac{3}{2}}}\right]\right)_{\epsilon} \\
\left(\mathbf{R}^{-}(\epsilon)\right)_{\epsilon} & =-\left(\frac{r^{2}+\epsilon^{2}}{\left(\sqrt{r^{2}+\epsilon^{2}}-|a|\right)^{2}}\left[\frac{9 a \epsilon^{2}}{\left(r^{2}+\epsilon^{2}\right)^{\frac{5}{2}}}-\frac{2 a \epsilon^{2}}{r^{2}\left(r^{2}+\epsilon^{2}\right)^{\frac{3}{2}}}\right]\right)_{\epsilon}
\end{aligned}
$$

Remark 3.6 Note that (i) on horizon $r=a$ Colombeau scalars $\left(\mathbf{R}^{ \pm}(\epsilon)\right)_{\epsilon}$ well defined and becomes to infinite large Colombeau generalized numbers

$$
\left\{\begin{array}{c}
\left(\mathbf{R}^{+}(\epsilon)\right)_{\epsilon}=\left(\frac{a^{2}+\epsilon^{2}}{\left(\sqrt{a^{2}+\epsilon^{2}}-|a|\right)^{2}}\left[\frac{9 a \epsilon^{2}}{\left(a^{2}+\epsilon^{2}\right)^{\frac{5}{2}}}-\frac{2 a \epsilon^{2}}{a^{2}\left(a^{2}+\epsilon^{2}\right)^{\frac{3}{2}}}\right]\right)_{\epsilon} \\
=7 a^{-2}\left(\epsilon^{-2}\right)_{\epsilon} \in \widetilde{\mathbb{R}} \\
-\left(\frac{a^{2}+\epsilon^{2}}{\left(\sqrt{a^{2}+\epsilon^{2}}-|a|\right)^{2}}\left[\frac{9 a \epsilon^{2}}{\left(a^{2}+\epsilon^{2}\right)^{\frac{5}{2}}}-\frac{2 a \epsilon^{2}}{r^{2}\left(r^{2}+\epsilon^{2}\right)^{\frac{3}{2}}}\right]\right)_{\epsilon} \\
=-7 a^{-2}\left(\epsilon^{-2}\right)_{\epsilon} \in \widetilde{\mathbb{R}}
\end{array}\right.
$$

(ii) for $r \neq$ a Colombeau scalars $\left(\mathbf{R}^{ \pm}(\epsilon)\right)_{\epsilon}$ well defined and becomes to infinite small Colombeau generalized numbers $\left(\mathbf{R}^{ \pm}(\epsilon)\right)_{\epsilon} \approx \pm\left(\epsilon^{2}\right)_{\epsilon}$.

Using now Eq. A2 one obtains that the Colombeau scalars $\left(\mathbf{R}^{ \pm \mu \nu}(\epsilon) \mathbf{R}_{\mu \nu}^{ \pm}(\epsilon)\right)_{\epsilon}$ in terms of Colombeau generalized functions $\left(A_{\epsilon}^{ \pm}(r)\right)_{\epsilon},\left(B_{\epsilon}^{ \pm}(r)\right)_{\epsilon},\left(C_{\epsilon}^{ \pm}(r)\right)_{\epsilon},\left(D_{\epsilon}^{ \pm}(r)\right)_{\epsilon}$ are expressed as

$$
\left\{\begin{array}{c}
\left(\mathbf{R}^{ \pm \mu \nu}(\epsilon) \mathbf{R}_{\mu \nu}^{ \pm}(\epsilon)\right)_{\epsilon}= \\
2\left[\frac { ( r ^ { 2 } + \epsilon ^ { 2 } ) ^ { 2 } } { ( \sqrt { r ^ { 2 } + \epsilon ^ { 2 } } - | a | ) ^ { 4 } } \left\{\left[\frac{5}{2}\left[\frac{3 a \epsilon^{2}}{\left(r^{2}+\epsilon^{2}\right)^{\frac{5}{2}}}\right]^{2}-\frac{2 a \epsilon^{2}}{r^{2}\left(r^{2}+\epsilon^{2}\right)^{\frac{3}{2}}}\right]+\right.\right. \\
\left.\left.2\left[\frac{3 a \epsilon^{2}}{\left(r^{2}+\epsilon^{2}\right)^{\frac{5}{2}}}+\frac{a \epsilon^{2}}{r^{2}\left(r^{2}+\epsilon^{2}\right)^{\frac{3}{2}}}\right]^{2}\right\}\right)_{\epsilon}
\end{array}\right.
$$

Remark 3.7 Note that (i) on horizon $\mathrm{r}=$ a Colombeau scalars $\left(\mathbf{R}^{ \pm \mu \nu}(\epsilon) \mathbf{R}_{\mu \nu}^{ \pm}(\epsilon)\right)_{\epsilon}$ well defined and becomes to infinite large Colombeau generalized numbers, (ii) for $r \neq a$ Colombeau scalars $\left(\mathbf{R}^{ \pm}(\epsilon)\right)_{\epsilon}$ well defined and becomes to infinite small Colombeau generalized numbers.

Using now Eq. A2 one obtains that the Colombeau scalars $\left(\mathbf{R}^{ \pm \rho \sigma \mu \nu}(\epsilon) \mathbf{R}_{\rho \sigma \mu \nu}^{ \pm}(\epsilon)\right)_{\epsilon}$ in terms of Colombeau generalized functions $\left(A_{\epsilon}^{ \pm}(r)\right)_{\epsilon},\left(B_{\epsilon}^{ \pm}(r)\right)_{\epsilon},\left(C_{\epsilon}^{ \pm}(r)\right)_{\epsilon},\left(D_{\epsilon}^{ \pm}(r)\right)_{\epsilon}$ are expressed as

$$
\begin{gathered}
\left(\mathbf{R}^{ \pm \rho \sigma \mu \nu}(\epsilon) \mathbf{R}_{\rho \sigma \mu \nu}^{ \pm}(\epsilon)\right)_{\epsilon}=\left(\frac{12 a^{2}}{\left(\sqrt{r^{2}+\epsilon^{2}}-|a|\right)^{6}}\left[1+\frac{a \epsilon^{2}}{\left(r^{2}+\epsilon^{2}\right)^{\frac{3}{2}}}\right]^{2} \mp\right. \\
\mp \frac{4 a^{2}}{\left(\sqrt{r^{2}+\epsilon^{2}}-|a|\right)^{5}}\left[1+\frac{a \epsilon^{2}}{\left(r^{2}+\epsilon^{2}\right)^{\frac{3}{2}}}\right]^{2}\left[\frac{2 \epsilon^{2}}{r^{2}\left(r^{2}+\epsilon^{2}\right)}+\frac{9 \epsilon^{2}}{\left(r^{2}+\epsilon^{2}\right)^{\frac{5}{2}}}\right]+ \\
\frac{a^{2}}{\left(\sqrt{r^{2}+\epsilon^{2}}-|a|\right)^{4}}\left[\frac{4 \epsilon^{4}}{r^{4}\left(r^{2}+\epsilon^{2}\right)}+\frac{81 \epsilon^{4}}{\left(r^{2}+\epsilon^{2}\right)^{3}}\right] .
\end{gathered}
$$

Remark 3.8 Note that (i) on horizon $\mathrm{r}=$ a Colombeau scalars $\left(\mathbf{R}^{ \pm \rho \sigma \mu \nu}(\epsilon) \mathbf{R}_{\rho \sigma \mu \nu}^{ \pm}(\epsilon)\right)_{\epsilon}$ well defined and becomes to infinite large Colombeau generalized numbers, (ii) for $r \neq a$ Colombeau scalars are 
finite

$$
\left(\mathbf{R}^{ \pm \rho \sigma \mu \nu}(\epsilon) \mathbf{R}_{\rho \sigma \mu \nu}^{ \pm}(\epsilon)\right)_{\epsilon}=\frac{12 a^{2}}{\left(r^{2}-|a|\right)^{6}}
$$

and tend to zero in the limit $r \rightarrow \infty$.

Remark 3.9 Note that under generalized transformatios such as

$$
d t=\left(d\left[\frac{\sqrt{r^{2}+\epsilon^{2}}-2 m}{r}\right] v_{\epsilon}^{+}\right)_{\epsilon}+\left(\frac{\sqrt{r^{2}+\epsilon^{2}}-2 m}{r} d v_{\epsilon}^{+}\right)_{\epsilon},
$$

and

$$
d t=\left(d\left[\frac{2 m-\sqrt{r^{2}+\epsilon^{2}}}{r}\right] v_{\epsilon}^{-}\right)_{\epsilon}+\left(\frac{2 m-\sqrt{r^{2}+\epsilon^{2}}}{r} d v_{\epsilon}^{-}\right)_{\epsilon},
$$

the metric given by Eq.(3.61)-Eq.(3.64) becomes to Colombeau metric of the form

$$
\left\{\begin{array}{c}
d s_{\varepsilon}^{ \pm 2}=\mp A^{ \pm}(r, \varepsilon)\left(d v_{\epsilon}^{ \pm}\right)^{2}-2 v_{\epsilon}^{ \pm} D_{2}^{ \pm}(r, \varepsilon) d v_{\epsilon}^{ \pm} d r+\left[B^{ \pm}(r, \varepsilon)+C_{1}^{ \pm}\left(v_{\epsilon}^{ \pm}, r, \varepsilon\right)\right](d r)^{2}+ \\
+B^{ \pm}(r, \varepsilon) r^{2}\left[(d \theta)^{2}+\sin ^{2} \theta(d \phi)^{2}\right] .
\end{array}\right.
$$

\section{Quantum Scalar Field Curved Distributional Spacetime}

\subsection{Canonical quantization in curved distributional spacetime}

Much of formalism can be explained with Colombeau generalized scalar field. The basic concepts and methods extend straightforwardly to distributional tensor and distributional spinor fields. To being with let's take a spacetime of arbitrary dimension $D$, with a metric $g_{\mu 3 b d}$ of signature $(+-\ldots-)$. The action for the Colombeau generalized scalar field $\left(\varphi_{\varepsilon}\right)_{\varepsilon} \in \mathcal{G}(M)$ is

$$
\left(S_{\varepsilon}\right)_{\varepsilon}=\left(\int_{M} d^{D} x \frac{1}{2} \sqrt{\left|g_{\varepsilon}\right|}\left(g_{\varepsilon}^{\mu \nu} \partial_{\mu} \varphi_{\varepsilon} \partial_{\nu} \varphi_{\varepsilon}\right)-\left(m^{2}+R_{\varepsilon}\right) \varphi_{\varepsilon}^{2}\right)_{\varepsilon} .
$$

The corresponding equation of motion is

$$
\left(\left[\square_{\varepsilon}+m^{2}+\xi R_{\varepsilon}\right] \varphi_{\varepsilon}\right)_{\varepsilon}=0, \varepsilon \in(0,1] .
$$

Here $\left(\square_{\varepsilon} \varphi_{\varepsilon}\right)_{\varepsilon}=\left(\left|g_{\varepsilon}\right|^{-1 / 2} \partial_{\mu}\left|g_{\varepsilon}\right|^{1 / 2} g_{\varepsilon}^{\mu \nu} \partial_{\mu} \varphi_{\varepsilon}\right)_{\varepsilon}$.

With $\hbar$ explicit, the mass $m$ should be replaced by $m / \hbar$. Separating out a time coordinate $x^{0}$, $x^{\mu}=\left(x^{0}, x^{i}\right), i=1,2,3$ we can write the action as

$$
\left(S_{\varepsilon}\right)_{\varepsilon}=\left(\int d x^{0} L_{\varepsilon}\right)_{\varepsilon},\left(L_{\varepsilon}\right)_{\varepsilon}=\left(\int d^{D-1} x_{\varepsilon}\right)_{\varepsilon} .
$$

The canonical momentum at a time $x^{0}$ is given by

$$
\left(\pi_{\varepsilon}(\underline{x})\right)_{\varepsilon}=\left(\delta L_{\varepsilon} / \delta\left(\partial_{0} \varphi_{\varepsilon}(\underline{x})\right)\right)_{\varepsilon}=\left(\left|h_{\varepsilon}\right|^{1 / 2} n^{\mu} \partial_{\mu} \varphi_{\varepsilon}(\underline{x})\right)_{\varepsilon},
$$

where $\underline{x}$ lables a point on a surface of constant $\mathrm{x}^{0}$, the $\mathrm{x}^{0}$ argument of $\left(\varphi_{\varepsilon}\right)_{\varepsilon}$ is suppressed, $n^{\mu}$ is the unit normal to the surface, and $\left(\left|h_{\varepsilon}\right|\right)_{\varepsilon}$ is the determinant of the induced spatial metric $\left(h_{i j}(\varepsilon)\right)_{\varepsilon}$. 
To quantize, the Colombeau generalized field $\left(\varphi_{\varepsilon}\right)_{\varepsilon}$ and its conjugate momentum $\left(\pi_{\varepsilon}(\underline{x})\right)_{\varepsilon}$ are now promoted to hermitian operators and required to satisfy the canonical commutation relation,

$$
\left(\left[\varphi_{\varepsilon}(\underline{x}), \pi_{\varepsilon}(\underline{y})\right]\right)_{\varepsilon}=i \hbar \delta^{D-1}(\underline{x}, \underline{y}), \varepsilon \in(0,1] .
$$

Here $\int d^{D-1} y \delta^{D-1}(\underline{x}, y) f(y)=f(\underline{x})$ for any scalar function $f \in D\left(\mathbb{R}^{3}\right)$, without the use of a metric volume element. We form now a conserved bracket from two complex Colombeau solutions to the scalar wave equation (4.2) by

$$
\left(\left\langle\varphi_{\varepsilon}, \phi_{\varepsilon}\right\rangle\right)_{\varepsilon}=\left(\int_{\Sigma} d \Sigma_{\mu} j_{\varepsilon}^{\mu}\right)_{\varepsilon}, \varepsilon \in(0,1] .
$$

where

$$
\left(j_{\varepsilon}^{\mu}\left(\varphi_{\varepsilon}, \phi_{\varepsilon}\right)\right)_{\varepsilon}=(i / \hbar)\left(\left|g_{\varepsilon}\right|^{1 / 2} g_{\varepsilon}^{\mu \nu}\left(\bar{\varphi}_{\varepsilon} \partial_{\nu} \phi_{\varepsilon}-\varphi_{\varepsilon} \partial_{\nu} \bar{\phi}_{\varepsilon}\right)\right)_{\varepsilon} .
$$

This bracket is called the generalized Klein-Gordon inner product, and $\left(\left\langle\phi_{\varepsilon}, \phi_{\varepsilon}\right\rangle\right)_{\varepsilon}$ the generalized Klein Gordon norm of $\left(\varphi_{\varepsilon}\right)_{\varepsilon}$. The generalized current density $\left(j_{\varepsilon}^{\mu}\left(\varphi_{\varepsilon}, \phi_{\varepsilon}\right)\right)_{\varepsilon}$ is divergenceless,i.e. $\left(\partial_{\mu} j_{\varepsilon}^{\mu}\left(\varphi_{\varepsilon}, \phi_{\varepsilon}\right)\right)_{\varepsilon}=0$ when the Colombeau generalized functions $\left(\varphi_{\varepsilon}\right)_{\varepsilon}$ and $\left(\phi_{\varepsilon}\right)_{\varepsilon}$ satisfy the KG equation (4.2), hence the value of the integral in (4.7) is independent of the spacelike surface $3 a 3$ over which it is evaluated, provided the functions vanish at spatial infinity. The generalized KG inner product satisfies the relations

$$
\left(\overline{\left\langle\varphi_{\varepsilon}, \phi_{\varepsilon}\right\rangle}\right)_{\varepsilon}=-\left(\left\langle\bar{\varphi}_{\varepsilon}, \bar{\phi}_{\varepsilon}\right\rangle\right)_{\varepsilon}=\left(\left\langle\phi_{\varepsilon}, \varphi_{\varepsilon}\right\rangle\right)_{\varepsilon}, \varepsilon \in(0,1] \text {. }
$$

We define now the annihilation operator associated with a complex Colombeau solution $\left(\phi_{\varepsilon}\right)_{\varepsilon}$ by the bracket of $\left(\phi_{\varepsilon}\right)_{\varepsilon}$ with the generalized field operator $\left(\varphi_{\varepsilon}\right)_{\varepsilon}$ :

$$
\left(a\left(\phi_{\varepsilon}\right)\right)_{\varepsilon}=\left(\left\langle\phi_{\varepsilon}, \varphi_{\varepsilon}\right\rangle\right)_{\varepsilon} .
$$

It follows from the hermiticity of $\left(\varphi_{\varepsilon}\right)_{\varepsilon}$ that the hermitian conjugate of $\left(a\left(\phi_{\varepsilon}\right)\right)_{\varepsilon}$ is given by

$$
\left(a^{\dagger}\left(\phi_{\varepsilon}\right)\right)_{\varepsilon}=-\left(a\left(\bar{\phi}_{\varepsilon}\right)\right)_{\varepsilon} .
$$

From Eq. (4.5) and CCR (4.6) one obtains

$$
\left(\left[a\left(\varphi_{\varepsilon}\right), a^{\dagger}\left(\phi_{\varepsilon}\right)\right]\right)_{\varepsilon}=\left(\left\langle\varphi_{\varepsilon}, \phi_{\varepsilon}\right\rangle\right)_{\varepsilon} .
$$

Note that from Eq. (4.11) follows

$$
\left(\left[a\left(\varphi_{\varepsilon}\right), a\left(\phi_{\varepsilon}\right)\right]\right)_{\varepsilon}=-\left(\left\langle\varphi_{\varepsilon}, \bar{\phi}_{\varepsilon}\right\rangle\right)_{\varepsilon},\left(\left[a^{\dagger}\left(\varphi_{\varepsilon}\right), a^{\dagger}\left(\phi_{\varepsilon}\right)\right]\right)_{\varepsilon}=-\left(\left\langle\bar{\varphi}_{\varepsilon}, \phi_{\varepsilon}\right\rangle\right) .
$$

Note that if $\left(\phi_{\varepsilon}\right)_{\varepsilon}$ is a positive norm solution with unit norm hf with, then $\left(a\left(\varphi_{\varepsilon}\right)\right)_{\varepsilon}$ and $a^{\dagger}\left(\varphi_{\varepsilon}\right)$ satisfy the commutation relation $\left(\left[a^{\dagger}\left(\varphi_{\varepsilon}\right), a\left(\phi_{\varepsilon}\right)\right]\right)_{\varepsilon}=1$. Suppose now that $|\Psi\rangle$ is a normalized quantum state satisfying $\left(a\left(\phi_{\varepsilon}\right)|\Psi\rangle\right)_{\varepsilon}=1$, then for each $n$, the state $|n, \Psi\rangle=\left((1 / \sqrt{ } n !)\left(a\left(\phi_{\varepsilon}\right)\right)^{n}|\Psi\rangle\right)_{\varepsilon}$ is a normalized eigenstate of the number operator $\left(N\left[\left(\phi_{\varepsilon}\right)\right]\right)_{\varepsilon}=\left(a^{\dagger}\left(\varphi_{\varepsilon}\right) a\left(\varphi_{\varepsilon}\right)\right)_{\varepsilon}$ with eigenvalue $n$. The span of all these states defines a Fock space of the distributional $\left(\varphi_{\varepsilon}\right)_{\varepsilon}$ - wavepacket " $n$-particle excitations" above the state $|\Psi\rangle$.If we want to construct the full Hilbert space of the field theory in curved distributional spacetime, how can we proceed? We should find a decomposition of the space of complex Colombeau solutions to the wave equation (4.2) $\mathbf{S}$ into a direct sum of a positive norm subspace $\mathbf{S}_{\mathbf{p}}$ and its complex conjugate $\mathbf{S}_{\mathbf{p}}$, such that all brackets between solutions from the two subspaces vanish. That is, we must find a direct sum decomposition: 


$$
\mathbf{S}=\mathbf{S}_{\mathbf{p}} \oplus \overline{\mathbf{S}_{\mathbf{p}}}
$$

such that

$$
\left(\left\langle\varphi_{\varepsilon}, \varphi_{\varepsilon}\right\rangle\right)_{\varepsilon}>0, \forall\left(\varphi_{\varepsilon}\right)_{\varepsilon} \in \mathbf{S}_{\mathbf{p}}
$$

and

$$
\left(\left\langle\varphi_{\varepsilon}, \phi_{\varepsilon}\right\rangle\right)_{\varepsilon}>0, \forall\left(\varphi_{\varepsilon}\right)_{\varepsilon},\left(\phi_{\varepsilon}\right)_{\varepsilon} \in \mathbf{S}_{\mathbf{p}}
$$

The condition (4.15) implies that each $\left(\varphi_{\varepsilon}\right)_{\varepsilon}$ in $\mathbf{S}_{\mathbf{p}}$ can be scaled to define its own harmonic oscillator sub-albegra. The second condition implies, according to (4.13), that the annihilators and creators for $\left(\varphi_{\varepsilon}\right)_{\varepsilon}$ and $\left(\phi_{\varepsilon}\right)_{\varepsilon}$ in the subspace $\mathbf{S}_{\mathbf{p}}$ commute amongst themselves:

$$
\left(\left[a\left(\varphi_{\varepsilon}\right), a\left(\phi_{\varepsilon}\right)\right]\right)_{\varepsilon}=\left(\left[a^{\dagger}\left(\varphi_{\varepsilon}\right), a^{\dagger}\left(\phi_{\varepsilon}\right)\right]\right)_{\varepsilon}=0 .
$$

Given such a decompostion a total Hilbert space $\mathbf{H}$ for the field theory can be defined as the space of finite norm sums of possibly infinitely many states of the form

$$
\left(a^{\dagger}\left(\phi_{1, \varepsilon}\right) \ldots a^{\dagger}\left(\phi_{n, \varepsilon}\right)|0\rangle\right)_{\varepsilon},
$$

where $|0\rangle$ is a state such that $\left(a\left(\phi_{n, \varepsilon}\right)|0\rangle\right)_{\varepsilon}=0$ for all $\left(\phi_{\varepsilon}\right)_{\varepsilon}$ in $\mathbf{S}_{\mathbf{p}}$. The state $|0\rangle$, as in classical case, is called a Fock vacuum and Hilbert space $\mathbf{H}$ is called a Fock space. The representation of the field operator on this Fock space is hermitian and satisfies the canonical commutation relations in sense of Colombeau generalized function.

\subsection{Defining distributional outgoing modes}

For illustration we consider the non-rotating, uncharged $d$-dimensional SAdS BH with a distributional line element

$$
\left(d s_{\varepsilon}^{2}\right)_{\varepsilon}=\left(-f_{\varepsilon} d t^{2}+f_{\varepsilon}^{-1} d r^{2}\right)_{\varepsilon}+r^{2} d \Omega_{d-2}^{2}, \varepsilon \in(0,1],
$$

where

$$
\left\{\begin{array}{c}
f_{\varepsilon} \neq 0, \varepsilon \in(0,1], \\
f_{\varepsilon=0}=1+\frac{r^{2}}{L^{2}}-\frac{r_{0}^{d-3}}{r^{d-3}},
\end{array}\right.
$$

where $d \Omega_{d-2}^{2}$ is the metric of the $(d-2)$-sphere, and the AdS curvature radius squared $L^{2}$ is related to the cosmological constant by $L^{2}=-(d-2)(d-1) / 2 \Lambda$. The parameter $r_{0}$ is proportional to the mass $M$ of the spacetime: $M=(d-2) A_{d-2} r_{0}^{d-3} / 16 \pi$, where $A_{d-2}=2 \pi^{(d-1) / 2} / \Gamma[(d-1) / 2]$. The distributional Schwarzschild geometry corresponds to $L \rightarrow \infty$. The corresponding equations of motion (4.2) for massless case are

$$
\begin{gathered}
\left(\nabla_{\mu} \nabla^{\mu} \varphi_{\varepsilon}\right)_{\varepsilon}=\frac{(d-2) \gamma}{4(d-1)}\left(R_{\varepsilon}\right)_{\varepsilon}, \\
\left(G_{\varepsilon, \mu \nu}\right)_{\varepsilon}+\Lambda\left(g_{\varepsilon, \mu \nu}\right)_{\varepsilon}=8 \pi G\left(T_{\varepsilon, \mu \nu}\right)_{\varepsilon}, \\
\left(T_{\varepsilon, \mu \nu}\right)_{\varepsilon} \sim \delta(x) .
\end{gathered}
$$

The time-independence and the spherical symmetry of the metric imply the canonical decomposition

$$
\left(\varphi_{\varepsilon}(t, r, \theta)\right)_{\varepsilon}=e^{-i \omega t}\left(\frac{\Psi_{l m, \varepsilon}(r) Y_{l m}(\theta)}{r^{(d-2) / 2}+\varepsilon}\right)_{\varepsilon},
$$

where $Y_{l m}(\theta)$ denotes the $d$-dimensional scalar spherical harmonics, satisfying 


$$
\Delta \Omega_{d-2} Y_{l m}(\theta)=-l(l+d-3) Y_{l m}(\theta),
$$

where $\Delta \Omega_{d-2}$ is the Laplace-Beltrami operator. Substituting the decomposition (4.22) into Eq. (6) one gets a radial wave equation

$$
\left(f_{\varepsilon}^{2} \frac{d^{2} \Psi_{l m, \varepsilon}(r)}{d r^{2}}+f_{\varepsilon} f_{\varepsilon}{ }^{\prime} \frac{d \Psi_{l m, \varepsilon}(r)}{d r}+\left(\omega^{2}-V_{l m, \varepsilon}(r)\right) \Psi_{l m, \varepsilon}(r)\right)_{\varepsilon}=0 .
$$

We define now a "tortoise" distributional coordinate $\left(r_{\varepsilon}^{*}\right)_{\varepsilon}=\left(r_{\varepsilon}^{*}(r)\right)_{\varepsilon}$ by the relation

$$
\left(\frac{d r_{\varepsilon}^{*}}{d r}\right)_{\varepsilon}=\left(f_{\varepsilon}^{-1}(r)\right)_{\varepsilon}
$$

By using a "tortoise" distributional coordinate the Eq.(4.24) can be written in the form of a Schrödinger equation with the potential $V_{l m, \varepsilon}(r)$

$$
\left(\frac{d \Psi_{\varepsilon}\left(r_{\varepsilon}^{*}\right)}{d r_{\varepsilon}^{*}}\right)_{\varepsilon}+\left(\left(\omega^{2}-V_{\varepsilon}\left(r_{\varepsilon}^{*}\right)\right) \Psi_{\varepsilon}\left(r_{\varepsilon}^{*}\right)\right)_{\varepsilon}=0
$$

Note that the tortoise distributional coordinate $\left(r_{\varepsilon}^{*}(r)\right)_{\varepsilon}$ becomes to infinite Colombeau constant $\left[\left(r_{\varepsilon}^{*}\left(r_{+}\right)\right)_{\varepsilon}\right]=\left[(\ln \varepsilon)_{\varepsilon}\right]$ at the horizon, i.e. as $r \rightarrow r_{+}$, but its behavior at infinity is strongly dependent on the cosmological constant: $\left[\left(r_{\varepsilon}^{*}\left(r_{+}\right)\right)_{\varepsilon}\right]=+\infty$ for asymptotically-flat spacetimes, and $\left[\left(r_{\varepsilon}^{*}\left(r_{+}\right)\right)_{\varepsilon}\right]=$ finite Colombeau constant for the $\operatorname{SAdS}_{d}$ geometry.

\subsubsection{Boundary conditions at the horizon of the distributional SAdS BH geometry}

For most spacetimes of interest the potential $\left(V_{\varepsilon}\left(r_{\varepsilon}^{*}(r)\right)\right)_{\varepsilon}=0$ as $r=r_{+}$, i.e. $\left(\left|r_{\varepsilon}^{*}(r)\right|\right)_{\varepsilon}=+\infty$, and in this limit solutions to the wave equation (4.26) behave as

$$
\left(\Psi_{\varepsilon}\left(t, r_{\varepsilon}^{*}\right)\right)_{\varepsilon} \sim\left(\exp \left[-i \omega\left(t \pm r_{\varepsilon}^{*}(r)\right)\right]\right)_{\varepsilon}, \text { as } r^{\sim} r_{+} .
$$

Note that classically nothing should leave the horizon and thus classically only ingoing modes (corresponding to a plus sign) should be present, i.e.

$$
\left(\Psi_{\varepsilon}\left(t, r_{\varepsilon}^{*}\right)\right)_{\varepsilon} \sim\left(\exp \left[-i \omega\left(t+r_{\varepsilon}^{*}(r)\right)\right]\right)_{\varepsilon}, \text { as } r^{\sim} r_{+} .
$$

Note that for non-extremal spacetimes, the tortoise coordinate tends to

$$
\left(r_{\varepsilon}^{*}(r)\right)_{\varepsilon}=\left(f_{\varepsilon}{ }^{-1}(r) d r\right)_{\varepsilon} \sim\left[\left(f_{\varepsilon}{ }^{\prime}\left(r_{+}\right)\right)_{\varepsilon}\right]^{-1}\left(\ln \left(\left|r-r_{+}\right|+\varepsilon\right)\right)_{\varepsilon} \text { as } r^{\sim} r_{+},
$$

where $\left(f_{\varepsilon}{ }^{\prime}\left(r_{+}\right)\right)_{\varepsilon}>0$. Therefore near the horizon, outgoing modes behave as

$$
\left\{\begin{array}{c}
\left(\exp \left[-i \omega\left(t-r_{\varepsilon}^{*}(r)\right)\right]\right)_{\varepsilon}=\left\{\left(\exp \left[-i \omega v_{\varepsilon}^{*}(t, r)\right]\right)_{\varepsilon}\right\}\left(\exp \left[-2 i \omega r_{\varepsilon}^{*}(r)\right]\right)_{\varepsilon}= \\
=\left\{\left(\exp \left[-i \omega v_{\varepsilon}^{*}(t, r)\right]\right)_{\varepsilon}\right\}\left\{\left(\left[\left|r-r_{+}\right|+\varepsilon\right]^{2 i \omega / f_{\varepsilon}^{\prime}\left(r_{+}\right)}\right)_{\varepsilon}\right\},
\end{array}\right.
$$

where $\left(v_{\varepsilon}^{*}(t, r)\right)_{\varepsilon}=t+\left(r_{\varepsilon}^{*}(r)\right)_{\varepsilon}$. Now Eq. (4.30) shows that outgoing modes is Colombeau generalized functions of class $\mathcal{G}(\mathbb{R})$.

\section{Energy-momentum Tensor Calculation by Using Colombeau Distributional Modes}

We shall assume now any distributional spacetime which is conformally static in both the asymptotic 
past and future. We will consider distributional spacetime which is conformally flat in the asymptotic past,i.e.

$$
\left\{\begin{array}{cc}
d s_{\varepsilon}^{2} \sim\left(f_{\varepsilon, \text { in }}^{2}\left(-d t^{2}+d \vec{x}^{2}\right)\right)_{\varepsilon} & \text { asymp.past } \\
d s_{\varepsilon}^{2} \sim\left(f_{\varepsilon, \text { out }}^{2}\left(-d t^{2}+h_{\varepsilon, i j} d x^{i} d x^{j}\right)\right)_{\varepsilon}, & \text { asymp.future }
\end{array}\right.
$$

where $\varepsilon \in(0,1]\left(f_{\varepsilon, \mathbf{J}}\right)_{\varepsilon}=\left(f_{\varepsilon, \mathbf{J}}(t, \vec{x})\right)_{\varepsilon}>0, \mathbf{J} \in\{$ in, out $\}$, are smooth functions and $h_{\varepsilon, i j}=$ $h_{\varepsilon, i j}(\vec{x}), i, j=1,2,3$, are the the components of an arbitrary distributional spatial metric. (Note that we use the same labels $t$ and $\vec{x}=\left(x^{1}, x^{2}, x^{3}\right)$ for coordinates in the asymptotic past and future only for simplicity; they are obviously defined on non-intersecting regions of the spacetime.) In each of these asymptotic regions the distributional field $\left(\Phi_{\varepsilon}\right)_{\varepsilon}$ can be written as $\left(\Phi_{\varepsilon}\right)_{\varepsilon}=\left(\tilde{\Phi}_{\varepsilon} / f_{\varepsilon, \mathbf{J}}\right)_{\varepsilon}$, where $\left(\tilde{\Phi}_{\varepsilon}\right)_{\varepsilon}$ satisfies

$$
-\left(\frac{\partial^{2}}{\partial t^{2}} \tilde{\Phi}\right)_{\varepsilon}=-\left(\Delta_{\varepsilon, \mathrm{J}} \tilde{\Phi}_{\varepsilon}\right)_{\varepsilon}+\left(V_{\varepsilon, \mathrm{J}} \tilde{\Phi}_{\varepsilon}\right)_{\varepsilon},
$$

where $\left(\Delta_{\varepsilon, \text { in }}\right)_{\varepsilon}$ is the flat Laplace operator, $\left(\Delta_{\varepsilon, \text { out }}\right)_{\varepsilon}$ is the Laplace operator associated with the spatial metric $\left(h_{\varepsilon i j}\right)_{\varepsilon}$, and the effective potential $V_{J}$ is given by

$$
\left\{\begin{array}{c}
\left(V_{\varepsilon, \mathbf{J}}\right)_{\varepsilon}=\left(\frac{\Delta_{\varepsilon \mathbf{J}} f_{\varepsilon, \mathbf{J}}}{f_{\varepsilon, \mathbf{J}}}\right)_{\varepsilon}+\left(f_{\varepsilon, \mathbf{J}}^{2}\left(m^{2}+\xi R_{\varepsilon}\right)\right)_{\varepsilon}= \\
(1-6 \xi)\left(\frac{\Delta_{\varepsilon \mathbf{J}} f_{\varepsilon, \mathbf{J}}}{f_{\varepsilon, \mathbf{J}}}\right)_{\varepsilon}+m^{2}\left(f_{\varepsilon, \mathbf{J}}^{2}\right)_{\varepsilon}+\xi\left(K_{\varepsilon, \mathbf{J}}\right)_{\varepsilon},
\end{array}\right.
$$

with $\left(K_{\varepsilon, \text { in }}\right)_{\varepsilon}=0, K_{\varepsilon, \text { out }}=K_{\varepsilon, \text { out }}(\vec{x})$ the scalar curvature associated with the spatial distributional metric $\left(h_{\varepsilon, i j}\right)_{\varepsilon}$.

We assume now this condition : (i) the massless $(\mathrm{m}=0)$ field with arbitrary coupling $\xi$ in spacetimes

which are asymptotically flat in the past and asymptotically static in the future, i.e. $f_{\text {in }}=1$ and $f_{\varepsilon, \text { out }}=f_{\varepsilon, \text { out }}(\vec{x})$, as those describing the formation of a static BH from matter initially scattered throughout space, and (ii) the massless, conformally coupled field ( $m=0$ and $\xi=1 / 6)$. With this assumptions for the potential, two different sets of positive-norm distributional modes, $\left(u_{\varepsilon, \vec{k}}^{(+)}\right)_{\varepsilon}$ and $\left(v_{\varepsilon, \alpha}^{(+)}\right)_{\varepsilon}$, can be naturally defined by the requirement that they are the solutions of Eq. (5.2) which satisfy the asymptotic conditions:

$$
\left.\left(u_{\varepsilon, \vec{k}}^{(+)}\right)_{\varepsilon \text { past }} \nsucceq 16 \pi^{3} \omega_{\vec{k}}\right)^{-1 / 2} e^{-i\left(\omega_{\vec{k}} t-\vec{k} \cdot \vec{x}\right)}\left(f_{\varepsilon, i n}^{-1}\right)_{\varepsilon}
$$

and

$$
\left(v_{\varepsilon, \alpha}^{(+)}\right)_{\varepsilon} \underset{\text { future }}{\asymp}\left(2 \varpi_{\alpha}\right)^{-1 / 2} e^{-i \varpi_{\alpha} t}\left(f_{\varepsilon, \text { out }}^{-1} F_{\varepsilon, \alpha}(\vec{x})\right)_{\varepsilon},
$$

where $\vec{k} \in \mathbb{R}^{3}, \omega_{\vec{k}}:=\|\vec{k}\|, \varpi_{\alpha}>0$, and $\left(F_{\varepsilon, \alpha}(\vec{x})\right)_{\varepsilon}$ are Colombeau solutions of

$$
\begin{gathered}
\left(\left[-\Delta_{\varepsilon, \text { out }}+V_{\varepsilon, \text { out }}(\vec{x})\right] F_{\varepsilon, \alpha}(\vec{x})\right)_{\varepsilon}=\varpi_{\alpha}^{2}\left(F_{\varepsilon, \alpha}(\vec{x})\right)_{\varepsilon} \\
\left.\left(F_{\varepsilon, \alpha}(\vec{x})\right)_{\varepsilon}\right|_{\varepsilon=0} \in C^{\infty}\left(\mathbb{R}^{3}\right)
\end{gathered}
$$

satisfying the normalization

$$
\left(\int_{\Sigma_{\text {out }}} d^{3} x \sqrt{h} F_{\varepsilon, \alpha}(\vec{x})^{*} F_{\varepsilon, \beta}(\vec{x})\right)_{\varepsilon}=\delta_{\alpha, \beta}
$$


on a Cauchy surface $\Sigma_{\text {out }}$ in the asymptotic future. Note that each $F_{\varepsilon, \alpha}, \varepsilon \in[0,1]$ can be chosen to be real without loss of generality. There are reasonable situations where the distributional modes $\left(v_{\varepsilon, \alpha}^{(+)}\right)_{\varepsilon}$, given in Eq. (5.5), together with distributional modes $\left(v_{\varepsilon, \alpha}^{(-)}\right)_{\varepsilon}$ fail to form a complete set of distributional normal modes. This happens whenever the operator $\left(\left[-\Delta_{\varepsilon, \text { out }}+V_{\varepsilon, \text { out }}(\vec{x})\right]\right)_{\varepsilon}$ in Eq. (5.2) happens to possess normalizable, i.e., satisfying Eq. (5.7) eigenfunctions with negative eigenvalues, $\varpi_{\alpha}^{2}=-\Omega_{\alpha}^{2}<0$. In this case, additional positive-norm modes $\left(w_{\varepsilon, \alpha}^{(+)}\right)_{\varepsilon}$ with the asymptotic behavior

$$
\left(w_{\varepsilon, \alpha}^{(+)}\right)_{\text {fyture }} \asymp\left(e^{\Omega_{\alpha} t-i \pi / 12}+e^{-\Omega_{\alpha} t+i \pi / 12}\right)\left(\frac{F_{\varepsilon, \alpha}(\vec{x})}{\sqrt{2 \Omega_{\alpha}} f_{\varepsilon, \text { out }}(\vec{x})}\right)_{\varepsilon}
$$

and their complex conjugates $\left(w_{\varepsilon, \alpha}^{(-)}\right)_{\varepsilon}$ are necessary in order to expand an arbitrary Colombeau solution of Eq. (5.1) As a direct consequence, at least some of the in-modes $\left(u_{\varepsilon, \vec{k}}^{( \pm)}\right)_{\varepsilon}$ (typically those with lown $\omega_{\vec{k}}$ ) eventually undergo an exponential growth. This asymptotic divergence is reflected on the unbounded increase of the vacuum fluctuations,

$$
\left(\left\langle\Phi_{\varepsilon}^{2}(\vec{x})\right\rangle\right)_{\text {future }} \underset{\widetilde{r}}{\asymp} \frac{\kappa e^{2 \bar{\Omega} t}}{2 \bar{\Omega}}\left[\left(\frac{\bar{F}_{\varepsilon}(\vec{x})}{f_{\varepsilon, \text { out }}(\vec{x})}\right)_{\varepsilon}\right]^{2}\left[1+\mathcal{O}\left(e^{-\epsilon t}\right)\right],
$$

where $\bar{F}(\vec{x})$ is the eigenfunction of Eq. (5.6) associated with the lowest negative eigenvalue allowed, $\varpi_{\alpha}^{2}=-\bar{\Omega}^{2}, \epsilon$ is some positive constant, and $\kappa$ is a dimensionless constant (typically of order unity) whose exact value depends globally on the spacetime structure (since it crucially depends on the projection of each $\left(u_{\varepsilon, \vec{k}}^{( \pm)}\right)_{\varepsilon}$ on the mode $\left(w_{\varepsilon, \alpha}^{( \pm)}\right)_{\varepsilon}$ whose $\varpi_{\alpha}^{2}=-\bar{\Omega}^{2} ; \kappa$ also depends on the initial state, here assumed to be the vacuum $|0\rangle_{i n}$ ). As one would expect, these wild quantum fluctuations give an important contribution to the vacuum energy stored in the field. In fact, the expectation value of its distributional energy-momentum tensor, $\left(\left\langle T_{\varepsilon, \mu \nu}(\vec{x})\right\rangle\right)_{\varepsilon}, \varepsilon \in(0,1]$, in the asymptotic future is found to be dominated by this exponential growth:

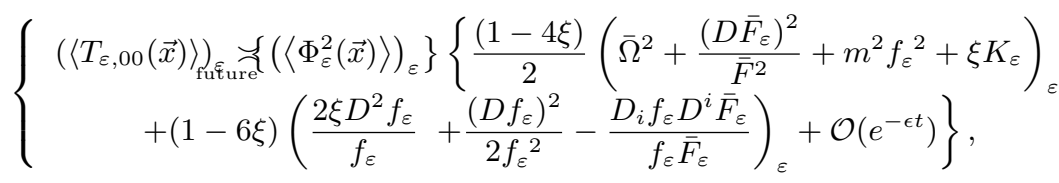

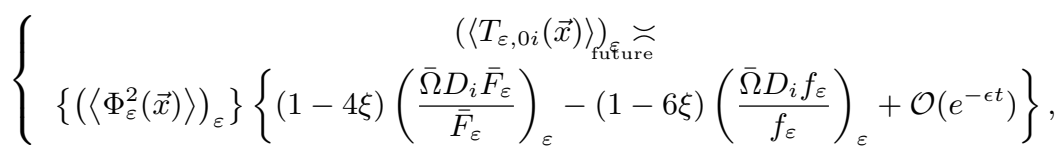

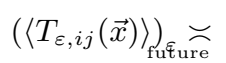

$$
\begin{aligned}
& \left\{\left(\left\langle\Phi_{\varepsilon}^{2}(\vec{x})\right\rangle\right)_{\varepsilon}\right\}\left\{(1-2 \xi)\left(\frac{D_{i} \bar{F}_{\varepsilon} D_{j} \bar{F}_{\varepsilon}}{\bar{F}_{\varepsilon}^{2}}\right)_{\varepsilon}-2 \xi\left(\frac{D_{i} D_{j} \bar{F}_{\varepsilon}}{F_{\varepsilon}}\right)_{\varepsilon}+\xi\left(\widetilde{R}_{\varepsilon, i j}\right)_{\varepsilon}\right. \\
& +\frac{(1-4 \xi) h_{i j}}{2}\left(\bar{\Omega}^{2}-\left(\frac{\left(D \bar{F}_{\varepsilon}\right)^{2}}{\bar{F}_{\varepsilon}^{2}}-m^{2} f_{\varepsilon}^{2}-\xi K_{\varepsilon}\right)_{\varepsilon}^{\varepsilon}\right) \\
& +(1-6 \xi)\left[\left(\frac{D_{i} f_{\varepsilon} D_{j} f_{\varepsilon}}{f_{\varepsilon}^{2}}-\frac{D_{i} f D_{j} \bar{F}}{f_{\varepsilon} \bar{F}_{\varepsilon}}-\frac{D_{j} f_{\varepsilon} D_{i} \bar{F}_{\varepsilon}}{f_{\varepsilon} \bar{F}_{\varepsilon}}\right)_{\varepsilon}^{\varepsilon}+\right. \\
& \left.+\left(h_{\varepsilon, i j}\right)_{\varepsilon}\left(\frac{2 \xi D^{2} f_{\varepsilon}}{f_{\varepsilon}}-\frac{\left(D f_{\varepsilon}\right)^{2}}{2 f_{\varepsilon}^{2}}+\frac{D_{k} f_{\varepsilon} D^{k} \bar{F}_{\varepsilon}}{f_{\varepsilon} \bar{F}_{\varepsilon}}\right)_{\varepsilon}\right]+\mathcal{O}\left(e^{-\epsilon t}\right),
\end{aligned}
$$

where $\mathrm{D}_{i}$ is the derivative operator compatible with the distributional metric $\left(h_{\varepsilon, i j}\right)_{\varepsilon}$ (so that $\left.\Delta_{\text {out }}=D^{2}\right),\left(\widetilde{R}_{\varepsilon, i j}\right)_{\varepsilon}$ is the associated distributional Ricci tensor so that $\left(K_{\varepsilon, \text { out }}\right)_{\varepsilon}=\left(h_{\varepsilon}^{i j} \widetilde{R}_{\varepsilon, i j}\right)_{\varepsilon}$, 
and we have omitted the subscript out in $\left(f_{\varepsilon, \text { out }}\right)_{\varepsilon}$ and $\left(K_{\varepsilon, \text { out }}\right)_{\varepsilon}$ for simplicity. The Eqs. (5.10-5.12), together with Eq. (5.9), imply that on time scales determined by $\bar{\Omega}^{-1}$, the vacuum fluctuations of the field should overcome any other classical source of energy, therefore taking control over the evolution of the background geometry through the semiclassical Einstein equations (in which $\left(\left\langle T_{\varepsilon, \mu \nu}\right\rangle\right)_{\varepsilon}$ is included as a source term for the distributional Einstein tensor). We are then confronted with a startling situation where the quantum fluctuations of a field, whose energy is usually negligible in comparison with classical energy components, are forced by the distributional background spacetime to play a dominant role. We are still left with the task of showing that there exist indeed wellbehaved distributional background spacetimes in which the operator $\left[\left(-\Delta_{\varepsilon, \text { out }}+V_{\varepsilon, \text { out }}(\vec{x})\right)_{\varepsilon}\right]$ possesses negative eigenvalues $\varpi_{\alpha}^{2}<0$, condition on which depends Eq. (5.9). Experience from quantum mechanics tells us that this typically occurs when $\left(V_{\varepsilon, \text { out }}\right)_{\varepsilon}$ gets sufficiently negative over a sufficiently large region. It is easy to see from Eq. (5.3) that, except for very special geometries (as the flat one), one can generally find appropriate values of $\xi \in \mathbb{R}$ which make $\left(V_{\varepsilon, \text { out }}\right)_{\varepsilon}$ as negative as would be necessary in order to guarantee the existence of negative eigenvalues. For distributional BH spacetime using Eq. (5.9)-Eq. (5.12) one obtains

$$
\begin{aligned}
& \left(\left\langle\Phi_{\varepsilon}^{2}(r)\right\rangle\right)_{\text {future }} \frac{\kappa e^{2 \bar{\Omega} t}}{2 \bar{\Omega}}\left[\left(\frac{r^{1 / 2} \bar{F}_{\varepsilon}(r)}{\left(\left(r-r_{+}\right)^{2}+\varepsilon^{2}\right)^{1 / 4}}\right)_{\varepsilon}\right]^{2}, r \rightarrow r_{+}
\end{aligned}
$$

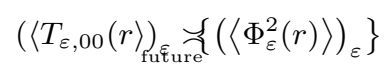

$$
\begin{aligned}
& \left\{\frac{(1-4 \xi)}{2}\left(\bar{\Omega}^{2}+\frac{\left(D \bar{F}_{\varepsilon}\left(r_{+}\right)\right)^{2}}{\bar{F}^{2}\left(r_{+}\right)}+m^{2}\left(\left(r-r_{+}\right)^{2}+\varepsilon^{2}\right)^{1 / 2}+\xi K_{\varepsilon}\right)_{\varepsilon}+(1-6 \xi)\right. \\
& \left.\left(-\frac{2 \xi D^{2} f_{\varepsilon}(r)}{\left(\left(r-r_{+}\right)^{2}+\varepsilon^{2}\right)^{1 / 4}}+\frac{\left(D f_{\varepsilon}(r)\right)^{2}}{\left(\left(r-r_{+}\right)^{2}+\varepsilon^{2}\right)^{1 / 4}}-\frac{D_{i} f_{\varepsilon}(r) D^{i} \bar{F}_{\varepsilon}}{\left(\left(r-r_{+}\right)^{2}+\varepsilon^{2}\right)^{1 / 4} \bar{F}_{\varepsilon}}\right)_{\varepsilon}\right\}, \\
& r \rightarrow r_{+}, f_{\varepsilon}(r)=\left(\left(r-r_{+}\right)^{2}+\varepsilon^{2}\right)^{1 / 4}
\end{aligned}
$$

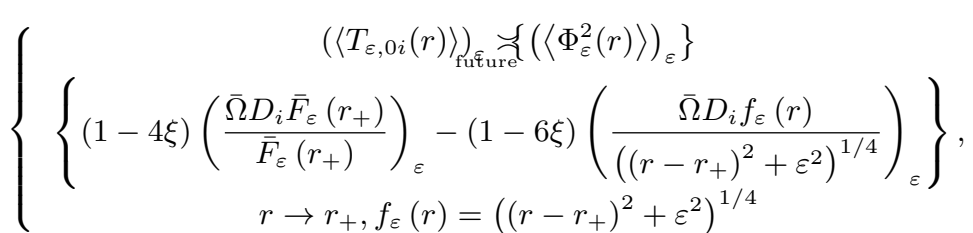

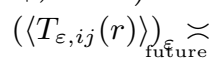

$$
\begin{aligned}
& \left\{\left(\left\langle\Phi_{\varepsilon}^{2}(r)\right\rangle\right)_{\varepsilon}\right\}\left\{(1-2 \xi)\left(\frac{D_{i} \bar{F}_{\varepsilon} D_{j} \bar{F}_{\varepsilon}}{\bar{F}_{\varepsilon}^{2}}\right)_{\varepsilon}-2 \xi\left(\frac{D_{i} D_{j} \bar{F}_{\varepsilon}}{F_{\varepsilon}}\right)_{\varepsilon}+\xi\left(\widetilde{R}_{\varepsilon, i j}\right)_{\varepsilon}+\right. \\
& \frac{(1-4 \xi) h_{i j}}{2}\left(\bar{\Omega}^{2}-\left(\frac{\left(D \bar{F}_{\varepsilon}\left(r_{+}\right)\right)^{2}}{\bar{F}_{\varepsilon}^{2}\left(r_{+}\right)}-m^{2}\left(\left(r-r_{+}\right)^{2}+\varepsilon^{2}\right)^{1 / 2}-\xi K_{\varepsilon}\right)_{\varepsilon}\right)+(1-6 \xi) \\
& {\left[\left(\frac{D_{i} f_{\varepsilon} D_{j} f_{\varepsilon}}{\left(\left(r-r_{+}\right)^{2}+\varepsilon^{2}\right)^{1 / 2}}-\frac{D_{i} f D_{j} \bar{F}}{\left(\left(r-r_{+}\right)^{2}+\varepsilon^{2}\right)^{1 / 4} \bar{F}_{\varepsilon}}-\frac{D_{j} f_{\varepsilon} D_{i} \bar{F}_{\varepsilon}}{\left(\left(r-r_{+}\right)^{2}+\varepsilon^{2}\right)^{1 / 2} \bar{F}_{\varepsilon}}\right)_{\varepsilon}+\right.} \\
& \left.\left(h_{\varepsilon, i j}\right)_{\varepsilon}\left(\frac{2 \xi D^{2} f_{\varepsilon}}{\left(\left(r-r_{+}\right)^{2}+\varepsilon^{2}\right)^{1 / 4}}-\frac{\left(D f_{\varepsilon}\right)^{2}}{2\left(\left(r-r_{+}\right)^{2}+\varepsilon^{2}\right)^{1 / 2}}+\frac{D_{k} f_{\varepsilon} D^{k} \bar{F}_{\varepsilon}}{\left(\left(r-r_{+}\right)^{2}+\varepsilon^{2}\right)^{1 / 4} \bar{F}_{\varepsilon}}\right){ }_{\varepsilon}\right] \text {, }
\end{aligned}
$$

Remark 5.1 Note that in spite of the unbounded growth atr $\rightarrow r_{+}$in Eq. (5.13)-Eq.

$\left(\left\langle T_{\varepsilon, \mu \nu}\right\rangle\right)_{\varepsilon}$ is covariantly conserved: $\left(\nabla_{\mu}\left\langle T_{\varepsilon, \nu}^{\mu}\right\rangle\right)_{\varepsilon}=0$. In the static case $\left(f_{\varepsilon, \text { out }}\right)_{\varepsilon}=\left(f_{\varepsilon, \text { out }}(\vec{x})\right)_{\varepsilon}$, for instance for distributional BH geometry, this implies that the total vacuum energy is kept constant, although it continuously flows from spatial regions where its density is negative to spatial regions 
where it is positive.

Remark 5.2 Note that the singular behavior at $r \rightarrow r_{+}$appearing in Eq. (5.13)-Eq. (5.16) leads only to asymptotic divergences, i.e. all the quantities remain finite everywhere except horizon.

\section{Distributional SAdS BH Spacetime-induced Vacuum Dominance}

\subsection{Adiabatic expansion of green finctions}

Using equation of motion Eq. (5.2) one can obtain corresponding distributional generalization of the canonical Green functions equations. In particular for the distributional propagator

one obtains directly

$$
i G_{\varepsilon}^{ \pm}\left(x, x^{\prime}\right)=\left\langle 0\left|T\left(\varphi_{\varepsilon}^{ \pm}(x) \varphi_{\varepsilon}^{ \pm}\left(x^{\prime}\right)\right)\right| 0\right\rangle, \varepsilon \in(0,1]
$$

$$
\left(\left[\square_{\varepsilon, x}+m^{2}+\xi \mathbf{R}^{ \pm}(x, \varepsilon)\right] G_{\varepsilon}^{ \pm}\left(x, x^{\prime}\right)\right)_{\varepsilon}=-\left[-g^{ \pm}(x, \varepsilon)\right]^{-1 / 2} \delta^{n}\left(x-x^{\prime}\right) .
$$

Special interest attaches to the short distance behaviour of the Green functions, such as $\left(G_{\varepsilon}^{ \pm}\left(x, x^{\prime}\right)\right)_{\varepsilon}$ in the limit $\left\|x-x^{\prime}\right\| \rightarrow 0$ with a fixed $\varepsilon \in(0,1]$. We obtan now an adiabatic expansion of $\left(G_{\varepsilon}^{ \pm}\left(x, x^{\prime}\right)\right)_{\varepsilon}$.Introducing Riemann normal coordinates $y^{\mu}$ for the point $x$, with origin at the point $\mathrm{x}^{\prime}$ we have expanding

$$
\begin{aligned}
& \left(g_{\mu v}^{ \pm}(x, \varepsilon)\right)_{\varepsilon}=\eta_{\mu v}+\frac{1}{3}\left[\left(\mathbf{R}_{\mu \alpha v \beta}^{ \pm}(\varepsilon)\right)_{\varepsilon}\right] y^{\alpha} y^{\beta}-\frac{1}{6}\left[\left(\mathbf{R}_{\mu \alpha v \beta ; \gamma}^{ \pm}(\varepsilon)\right)_{\varepsilon}\right] y^{\alpha} y^{\beta} y^{\gamma}+ \\
& +\left[\frac{1}{20}\left(\mathbf{R}_{\mu \alpha v \beta ; \gamma \delta}^{ \pm}(\varepsilon)\right)_{\varepsilon}+\frac{2}{45}\left[\left(\mathbf{R}_{\alpha \mu \beta \lambda}^{ \pm}(\varepsilon)\right)_{\varepsilon}\right]\left(\mathbf{R}_{\gamma v \delta}^{ \pm \lambda}(\varepsilon)\right)_{\varepsilon}\right] y^{\alpha} y^{\beta} y^{\gamma} y^{\delta}+\ldots
\end{aligned}
$$

where $\eta_{\mu v}$ is the Minkowski metric tensor, and the coefficients are all evaluated at $y=0$. Defining now

$$
\left(\stackrel{ \pm}{\varepsilon}^{ \pm}\left(x, x^{\prime}\right)\right)_{\varepsilon}=\left[\left(\left(-g_{\mu v}^{ \pm}(x, \varepsilon)\right)^{1 / 4}\right)_{\varepsilon}\right]\left(G_{\varepsilon}^{ \pm}\left(x, x^{\prime}\right)\right)_{\varepsilon}
$$

and its Colombeau-Fourier transform by

$$
\left(\frac{ \pm}{\varepsilon}\left(x, x^{\prime}\right)\right)_{\varepsilon}=(2 \pi)^{-n}\left(d^{n} k e_{\varepsilon}^{-i k y \pm}(k)\right)_{\varepsilon},
$$

where $\mathrm{ky}=\eta^{\alpha \beta} k_{\alpha} y_{\beta}$, one can work in a sort of localized momentum space. Expanding (6.2) in normal coordinates and converting to $k$-space, $\left(\frac{ \pm}{\varepsilon}(k)\right)_{\varepsilon}$ can readily be solved by iteration to any adiabatic order. The result to adiabatic order four (i.e., four derivatives of the metric) is

$$
\begin{gathered}
\left({ }_{\varepsilon}^{ \pm}(k)\right)_{\varepsilon}=\left(k^{2}-m^{2}\right)^{-1}-\left(\frac{1}{6}-\xi\right)\left(k^{2}-m^{2}\right)^{-2}\left(\mathbf{R}^{ \pm}(\varepsilon)\right)_{\varepsilon}+ \\
\frac{i}{2}\left(\frac{1}{6}-\xi\right) \partial^{\alpha}\left(k^{2}-m^{2}\right)^{-2}\left(\mathbf{R}_{; \alpha}^{ \pm}(\varepsilon)\right)_{\varepsilon}-\frac{1}{3}\left[\left(a_{\alpha \beta}^{ \pm}(\varepsilon)\right)_{\varepsilon}\right] \partial^{\alpha} \partial^{\beta}\left(k^{2}-m^{2}\right)^{-2} \\
+\left[\left(\frac{1}{6}-\xi\right)^{2}\left(\mathbf{R}^{ \pm 2}(\varepsilon)\right)_{\varepsilon}+\frac{2}{3}\left(a_{\lambda}^{ \pm \lambda}(\varepsilon)\right)_{\varepsilon}\right]\left(k^{2}-m^{2}\right)^{-3}
\end{gathered}
$$

where $\partial_{\alpha}=\partial / \partial k^{\alpha}$, 


$$
\begin{gathered}
\left(a_{\alpha \beta}^{ \pm}(\varepsilon)\right)_{\varepsilon} \asymp\left(\frac{1}{2}-\xi\right)\left(\mathbf{R}_{; \alpha \beta}^{ \pm}(\varepsilon)\right)_{\varepsilon}+\frac{1}{120}\left(\mathbf{R}_{; \alpha \beta}^{ \pm}(\varepsilon)\right)_{\varepsilon}-\frac{1}{140}\left(\mathbf{R}_{\alpha \beta ; \lambda}^{ \pm}{ }^{\lambda}(\varepsilon)\right)_{\varepsilon}- \\
-\frac{1}{30}\left[\left(\mathbf{R}_{\alpha}^{ \pm \lambda}(\varepsilon)\right)_{\varepsilon}\right]\left(\mathbf{R}_{\lambda \beta}^{ \pm}(\varepsilon)\right)_{\varepsilon}+\frac{1}{60}\left[\left(\mathbf{R}_{\alpha \beta}^{ \pm \kappa}{ }_{\alpha}(\varepsilon)\right)_{\varepsilon}\right]\left(\mathbf{R}_{\kappa \lambda}^{ \pm}(\varepsilon)\right)_{\varepsilon}+ \\
+\frac{1}{60}\left[\left(\mathbf{R}_{\alpha}^{ \pm \lambda \mu \kappa}(\varepsilon)\right)_{\varepsilon}\right]\left(\mathbf{R}_{\lambda \mu \kappa \beta}^{ \pm}(\varepsilon)\right)_{\varepsilon}
\end{gathered}
$$

and we are using the symbol $\asymp$ to indicate that this is an asymptotic expansion. One ensures that Eq. (6.5) represents a time-ordered product by performing the $k^{0}$ integral along the appropriate contour in Pic. 3. This is equivalent to replacing $m^{2}$ by $m^{2}-i \epsilon$. Similarly, the adiabatic expansions of other Green functions can be obtained by using the other contours in Pic. 3. Substituting Eq. (6.6) into Eq.(6.5) gives

$$
\left\{\begin{array}{c}
\left(\mathscr{L}_{\varepsilon}^{ \pm}\left(x, x^{\prime}\right)\right)_{\varepsilon}=(2 \pi)^{-n} \times \\
\left(\int d^{n} k e^{-i k y}\left[a_{0}^{ \pm}\left(x, x^{\prime} ; \varepsilon\right)+a_{1}^{ \pm}\left(x, x^{\prime} ; \varepsilon\right)\left(-\frac{\partial}{\partial m^{2}}\right)+a_{2}^{ \pm}\left(x, x^{\prime} ; \varepsilon\right)\left(\frac{\partial}{\partial m^{2}}\right)^{2}\right]\left(k^{2}-m^{2}\right)^{-1}\right)_{\varepsilon},
\end{array}\right.
$$

where

$$
\left(a_{0}^{ \pm}\left(x, x^{\prime} ; \varepsilon\right)\right)_{\varepsilon}=1
$$

and, to adiabatic order 4 ,

$$
\left\{\begin{array}{c}
\left(a_{1}^{ \pm}\left(x, x^{\prime} ; \varepsilon\right)\right)_{\varepsilon}= \\
\left(\frac{1}{6}-\xi\right)\left(\mathbf{R}^{ \pm}(\varepsilon)\right)_{\varepsilon}-\frac{i}{2}\left(\frac{1}{6}-\xi\right)\left[\left(\mathbf{R}_{; \alpha}^{ \pm}(\varepsilon)\right)_{\varepsilon}\right] y^{\alpha}-\frac{1}{3}\left[\left(a_{\alpha \beta}^{ \pm}(\varepsilon)\right)_{\varepsilon}\right] y^{\alpha} y^{\beta} \\
\left(a_{2}^{ \pm}\left(x, x^{\prime} ; \varepsilon\right)\right)_{\varepsilon}=\frac{1}{2}\left(\frac{1}{6}-\xi\right)\left(\mathbf{R}^{ \pm 2}(\varepsilon)\right)_{\varepsilon}+\frac{1}{3}\left(a_{\lambda}^{ \pm \lambda}(\varepsilon)\right)_{\varepsilon}
\end{array}\right.
$$

with all geometric quantities on the right-hand side of Eq. (6.10) evaluated at $x^{\prime}$.

If one uses the canonical integral representation

$$
\left(k^{2}-m^{2}+i \epsilon\right)^{-1}=-i \int_{0}^{\infty} d s e^{i s\left(k^{2}-m^{2}+i \epsilon\right)}
$$

in Eq.(6.8), then the $\mathrm{d}^{n} k$ integration may be interchanged with the $d s$ integration, and performed explicitly to yield (dropping the $i \epsilon$ )

$$
\begin{gathered}
\left(\mathscr{L}_{\varepsilon}^{ \pm}\left(x, x^{\prime}\right)\right)_{\varepsilon}=-i(4 \pi)^{-n / 2}\left(\int_{0}^{\infty} i d s(i s)^{-n / 2} \exp \left[-i m^{2} s+\frac{\sigma\left(x, x^{\prime}\right)}{2 i s}\right] \mathcal{F}_{\varepsilon}^{ \pm}\left(x, x^{\prime} ; i s\right)\right)_{\varepsilon} \\
\sigma\left(x, x^{\prime}\right)=\frac{1}{2} y_{\alpha} y^{\alpha} .
\end{gathered}
$$

The function $\sigma\left(x, x^{\prime}\right)$ which is one-half of the square of the proper distance between $x$ and $x^{\prime}$, while the function $\left(\varepsilon\left(x, x^{\prime} ; i s\right)\right)_{\varepsilon}$ has the following asymptotic adiabatic expansion

$$
\left({ }_{\varepsilon}^{ \pm}\left(x, x^{\prime} ; i s\right)\right)_{\varepsilon} \asymp\left(a_{0}^{ \pm}\left(x, x^{\prime} ; \varepsilon\right)\right)_{\varepsilon}+i s\left(a_{1}^{ \pm}\left(x, x^{\prime} ; \varepsilon\right)\right)_{\varepsilon}+(i s)^{2}\left(a_{2}^{ \pm}\left(x, x^{\prime} ; \varepsilon\right)\right)_{\varepsilon}+\ldots
$$

Using Eq. (6.4), equation (6.12) gives a representation of $\left(G_{\varepsilon}^{ \pm}\left(x, x^{\prime}\right)\right)_{\varepsilon}$ :

$$
\left\{\begin{array}{c}
\left(G_{\varepsilon}^{ \pm}\left(x, x^{\prime}\right)\right)_{\varepsilon}= \\
-i(4 \pi)^{-n / 2}\left(\left[\left(\Delta_{ \pm}^{1 / 2}\left(x, x^{\prime} ; \varepsilon\right)\right)_{\varepsilon}\right] \int_{0}^{\infty} i d s(i s)^{-n / 2} \exp \left[-i m^{2} s+\frac{\sigma\left(x, x^{\prime}\right)}{2 i s}\right] \mathcal{F}_{\varepsilon}\left(x, x^{\prime} ; i s\right)\right)_{\varepsilon}
\end{array}\right.
$$

where $\left(\Delta_{ \pm}\left(x, x^{\prime} ; \varepsilon\right)\right)_{\varepsilon}$ is the distributional Van Vleck determinant 


$$
\left(\Delta_{ \pm}\left(x, x^{\prime} ; \varepsilon\right)\right)_{\varepsilon}=-\operatorname{det}\left[\partial_{\mu} \partial_{\nu} \sigma\left(x, x^{\prime}\right)\right]\left(\left[g^{ \pm}(x, \varepsilon) g^{ \pm}\left(x^{\prime}, \varepsilon\right)\right]^{-1 / 2}\right)_{\varepsilon} .
$$

In the normal coordinates about $x^{\prime}$ that we are currently using, $\left(\Delta_{ \pm}\left(x, x^{\prime} ; \varepsilon\right)\right)_{\varepsilon}$ reduces to $\left(\left[-g^{ \pm}(x, \varepsilon)\right]^{-1 / 2}\right)_{\varepsilon}$.

The full asymptotic expansion of $\left({ }_{\varepsilon}^{ \pm}\left(x, x^{\prime} ; i s\right)\right)_{\varepsilon}$ to all adiabatic orders is

$$
\left({ }_{\varepsilon}^{ \pm}\left(x, x^{\prime} ; i s\right)\right)_{\varepsilon} \asymp \sum_{j=0}^{\infty}(i s)^{j}\left(a_{2}^{ \pm}\left(x, x^{\prime} ; \varepsilon\right)\right)_{\varepsilon}
$$

with $\left(a_{0}^{ \pm}\left(x, x^{\prime} ; \varepsilon\right)\right)_{\varepsilon}=1$, the other $\left(a_{j}^{ \pm}\left(x, x^{\prime} ; \varepsilon\right)\right)_{\varepsilon}$ being given by canonical recursion relations which enable their adiabatic expansions to be obtained. The expansions (6.13) and (6.16) are, however, only asymptotic approximations in the limit of large adiabatic parameter $T$.

If (6.16) is substituted into (6.14) the integral can be performed to give the adiabatic expansion of the Feynman propagator in coordinate space:

$$
\begin{aligned}
\left(G_{\varepsilon}^{ \pm}\left(x, x^{\prime}\right)\right)_{\varepsilon} & =-(4 \pi i)^{-n / 2}\left(\Delta_{ \pm}^{1 / 2}\left(x, x^{\prime} ; \varepsilon\right) \sum_{j=0}^{\infty} a_{j}^{ \pm}\left(x, x^{\prime} ; \varepsilon\right)\left(-\frac{\partial}{\partial m^{2}}\right)^{j} \times\right. \\
& \left.\times\left[\left(-\frac{2 m^{2}}{\sigma}\right)^{\frac{n-2}{4}} H_{(n-2) / 2}^{(2)}\left(\left(2 m^{2} \sigma\right)^{\frac{1}{2}}\right)\right]\right)_{\varepsilon},
\end{aligned}
$$

in which, strictly, a small imaginary part i $\epsilon$ should be subtracted from $\sigma$. Since we have not imposed global boundary conditions on the distributional Green function Colombeau solution of (6.2), the expansion (6.17) does not determine the particular vacuum state in (6.1). In particular, the " $i \epsilon$ " in the expansion of $\left(G_{\varepsilon}^{ \pm}\left(x, x^{\prime}\right)\right)_{\varepsilon}$ only ensures that (6.17) represents the expectation value, in some set of states, of a time-ordered product of fields. Under some circumstances the use of " $i \epsilon$ " in the exact representation (6.14) may give additional information concerning the global nature of the states

\subsection{Effective action for the quantum matter fields in curved distribu- tional spacetime}

As in classical case one can obtain Colombeau generalized quantity $\left(W_{\varepsilon}\right)_{\varepsilon}$, called the effective action for the quantum matter fields in curved distributional spcetime, which, when functionally differentiated, yields

$$
\left(\frac{2}{(-g(\varepsilon))^{\frac{1}{2}}} \frac{\delta W_{\varepsilon}}{\delta g^{\mu \nu}(\varepsilon)}\right)_{\varepsilon}=\left(\left\langle\mathbf{T}_{\mu \nu}(\varepsilon)\right\rangle\right)_{\varepsilon}
$$

To discover the structure of $\left(W_{\varepsilon}\right)_{\varepsilon}$, let us return to first principles, recalling the Colombeau pathintegral quantization procedure such as developed in [1], [2]. Our notation will imply a treatment for the scalar field, but the formal manipulations are identical for fields of higher spins. Note that the generating functional

$$
\left(Z_{\varepsilon}\left[\mathbf{J}_{\varepsilon}\right]\right)_{\varepsilon}=\left(D\left[\varphi_{\varepsilon}\right] \exp \left\{i S_{\mathbf{m}}(\varepsilon)+i \mathbf{J}_{\varepsilon}(x) \varphi_{\varepsilon}(x) d^{n} x\right\}\right)_{\varepsilon}
$$

was interpreted physically as the vacuum persistence amplitude $\left(\left\langle\mathbf{o u t}_{\varepsilon}, 0 \mid 0, \mathbf{i n}_{\varepsilon}\right\rangle\right)_{\varepsilon}$. The presence of the external distributional current density $\left(\mathbf{J}_{\varepsilon}\right)_{\varepsilon}$ can cause the initial vacuum state $\left(\left|0, \mathbf{i n}_{\varepsilon}\right\rangle\right)_{\varepsilon}$ to be unstable, i.e., it can bring about the production of particles. In flat space, in the limit $\left(\mathbf{J}_{\varepsilon}\right)_{\varepsilon}=0$, no particles are produced, and one have the normalization condition 


$$
\left(Z_{\varepsilon}[0]\right)_{\varepsilon}=\left.\left(D\left[\varphi_{\varepsilon}\right] \exp \left\{i S_{\mathbf{m}}(\varepsilon)+i \mathbf{J}_{\varepsilon}(x) \varphi_{\varepsilon}(x) d^{n} x\right\}\right)_{\varepsilon}\right|_{\mathbf{J}=0}=\left(\left\langle 0_{\varepsilon} \mid 0_{\varepsilon}\right\rangle\right)_{\varepsilon}=1 .
$$

However, when distributional spacetime is curved, we have seen that, in general,

$$
\left.\left(\mid 0, \text { out }_{\varepsilon}\right\rangle\right)_{\varepsilon} \neq\left(\left|0, \mathbf{i n}_{\varepsilon}\right\rangle\right)_{\varepsilon},
$$

even in the absence of source currents J. Hence (6.19) will no longer apply. Path-integral quantization still works in curved distributional spacetime; one simply treats $\left(S_{\mathbf{m}}(\varepsilon)\right)_{\varepsilon}$ in $(6.19)$ as the curved distributional spacetime matter action, and $\left(\mathbf{J}_{\varepsilon}(x)\right)_{\varepsilon}$ as a current density (a scalar density in the case of scalar fields). One can thus set $\mathbf{J}_{\varepsilon}=0$ in (6.19) and examine the variation of $\left(Z_{\varepsilon}[0]\right)_{\varepsilon}$ :

$$
\left(\delta Z_{\varepsilon}[0]\right)_{\varepsilon}=i D\left[\varphi_{\varepsilon}\right] \delta S_{\mathbf{m}}(\varepsilon) \exp \left[i S_{\mathbf{m}}\left(\varphi_{\varepsilon} ; \varepsilon\right)\right]=i\left(\left\langle\mathbf{o u t}_{\varepsilon}, 0\left|\delta S_{\mathbf{m}}(\varepsilon)\right| 0, \mathbf{i n}_{\varepsilon}\right\rangle\right)_{\varepsilon} .
$$

Note that

$$
\left(\frac{2}{(-g(\varepsilon))^{\frac{1}{2}}} \frac{\delta S_{\mathbf{m}}(\varepsilon)}{\delta g^{\mu \nu}(\varepsilon)}\right)_{\varepsilon}=\left(\mathbf{T}_{\mu \nu}(\varepsilon)\right)_{\varepsilon} .
$$

From (6.22) and (6.23) one obtains directly

$$
\left(\frac{2}{(-g(\varepsilon))^{\frac{1}{2}}} \frac{\delta Z_{\varepsilon}[0]}{\delta g^{\mu \nu}(\varepsilon)}\right)_{\varepsilon}=i\left(\left\langle\text { out }_{\varepsilon}, 0\left|\mathbf{T}_{\mu \nu}(\varepsilon)\right| 0, \mathbf{i n}_{\varepsilon}\right\rangle\right)_{\varepsilon}
$$

Noting that the matter action $S_{\mathbf{m}}(\varepsilon)$ appears exponentiated in (6.19), one obtains directly

$$
Z_{\varepsilon}[0]=\left(\exp \left(i W_{\varepsilon}\right)\right)_{\varepsilon}
$$

and

$$
\left(\exp \left(W_{\varepsilon}\right)\right)_{\varepsilon}=-i\left(\ln \left\langle\mathbf{o u t}_{\varepsilon}, 0 \mid 0, \mathbf{i n}_{\varepsilon}\right\rangle\right)_{\varepsilon} .
$$

Following canonical calculation one obtains

$$
\left(Z_{\varepsilon}^{ \pm}[0]\right)_{\varepsilon} \propto\left(\left[\operatorname{det}\left(-G_{\varepsilon}^{ \pm}\left(x, x^{\prime}\right)\right)\right]^{\frac{1}{2}}\right)_{\varepsilon},
$$

where the proportionality constant is metric-independent and can be ignored. Thus we obtain

$$
\left(W_{\varepsilon}^{ \pm}\right)_{\varepsilon}=-i\left(\ln Z_{\varepsilon}^{ \pm}[0]\right)_{\varepsilon}=-\frac{i}{2}\left(\operatorname{tr}\left[\ln \left(-\hat{G}_{\varepsilon}^{ \pm}\right)\right]\right)_{\varepsilon} .
$$

In (6.28) $\left(\hat{G}_{\varepsilon}^{ \pm}\right)_{\varepsilon}$ is to be interpreted as an Colombeau generalized operator which acts on an linear space $\Im$ of generalized vectors $|x\rangle$, normalized by

$$
\left(\left\langle x \mid x^{\prime}\right\rangle\right)_{\varepsilon}=\delta\left(x-x^{\prime}\right)\left(\left[-g^{ \pm}(x, \varepsilon)\right]^{-\frac{1}{2}}\right)_{\varepsilon}
$$

in such a way that

$$
\left(G_{\varepsilon}^{ \pm}\left(x, x^{\prime}\right)\right)_{\varepsilon}=\left(\left\langle x\left|\hat{G}_{\varepsilon}^{ \pm}\right| x^{\prime}\right\rangle\right)_{\varepsilon} .
$$

Remark 6.1 Note that the trace $\operatorname{tr}[]$ of an Colombeau generalized operator $\left(\Re_{\varepsilon}\right)_{\varepsilon}$ which acts on a linear space $\Im$, is defined by

$$
\left(\operatorname{tr}\left[\Re_{\varepsilon}\right]\right)_{\varepsilon}=\left(\int d^{n} x\left[-g^{ \pm}(x, \varepsilon)\right]^{\frac{1}{2}} \Re_{x x ; \varepsilon}\right)_{\varepsilon}=\left(\int d^{n} x\left[-g^{ \pm}(x, \varepsilon)\right]^{\frac{1}{2}}\left\langle x\left|\Re_{x x ; \varepsilon}\right| x^{\prime}\right\rangle\right)_{\varepsilon} .
$$


Writing now the Colombeau generalized operator $\left(\hat{G}_{\varepsilon}^{ \pm}\right)_{\varepsilon}$ as

$$
\left(\hat{G}_{\varepsilon}^{ \pm}\right)_{\varepsilon}=-\left(\mathcal{F}_{\varepsilon}^{ \pm-1}\right)_{\varepsilon}=-i\left(\int_{0}^{\infty} d s \exp \left[-s \mathcal{F}_{\varepsilon}^{ \pm}\right]\right)_{\varepsilon}
$$

by Eq.(6.14) one obtains

$$
\left\{\begin{array}{c}
\left(\left\langle x\left|\exp \left[-s \mathcal{F}_{\varepsilon}^{ \pm}\right]\right| x^{\prime}\right\rangle\right)= \\
i(4 \pi)^{-n / 2}\left[\left(\Delta_{ \pm}^{1 / 2}\left(x, x^{\prime} ; \varepsilon\right)\right)_{\varepsilon}\right] \exp \left[-i m^{2} s+\frac{\sigma\left(x, x^{\prime}\right)}{2 i s}\right] \mathcal{F}_{\varepsilon}^{ \pm}\left(x, x^{\prime} ; i s\right)(i s)^{-n / 2}
\end{array}\right.
$$

Now, assuming $(\varepsilon)_{\varepsilon}$ to have a small negative imaginary part, we obtain

$$
\left(\int_{\Lambda}^{\infty} d s(i s)^{-1} i \exp \left[-s \mathcal{F}_{\varepsilon}^{ \pm}\right]\right)_{\varepsilon}=\left(E i\left(-i \Lambda \mathcal{F}_{\varepsilon}^{ \pm}\right)\right)_{\varepsilon},
$$

where $E i(x)$ is the exponential integral function.

Remark 6.2 Note that for $x \rightarrow 0$

$E i(x)=\gamma+\ln (-x)+O(x)$,

where $\gamma$ is the Euler's constant. Substituting now (6.35) into (6.34) and letting $\Lambda \rightarrow 0$ we obtain

$$
\left(\ln \left(-\hat{G}_{\varepsilon}^{ \pm}\right)\right)_{\varepsilon}=-\left(\ln \left(\mathcal{F}_{\varepsilon}\right)\right)_{\varepsilon}=\left(\int_{0}^{\infty} d s \exp \left[-s \mathcal{F}_{\varepsilon}^{ \pm}\right](i s)^{-1}\right)_{\varepsilon},
$$

which is correct up to the addition of a metric-independent infinite large Colombeau constant $\Omega \in \widetilde{\mathbb{R}}$ that can be ignored in what follows. Thus, in the generalized De Witt-Schwinger representation (6.33) or (6.14) we have

$$
\left(\left\langle x\left|\ln \left(-\hat{G}_{\varepsilon}^{ \pm}\right)\right| x^{\prime}\right\rangle\right)_{\varepsilon}=\left(\int_{m^{2}}^{\infty} G_{\varepsilon}^{ \pm}\left(x, x^{\prime} ; m^{2}\right) d m^{2}\right)_{\varepsilon},
$$

where the integral with respect to $m^{2}$ brings down the extra power of $(\text { is })^{-1}$ that appears in Eq. (6.36). Returning now to the expression (6.28) for $\left(W_{\varepsilon}^{ \pm}\right)_{\varepsilon}$ using Eq. (6.37) and Eq. (6.31) we get.

$$
\left(W_{\varepsilon}^{ \pm}\right)_{\varepsilon}=\frac{i}{2}\left[\left(\int d^{n} x\left[-g^{ \pm}(x, \varepsilon)\right]^{\frac{1}{2}}\right)_{\varepsilon}\right]\left(\lim _{x \rightarrow x^{\prime}} \int_{m^{2}}^{\infty} G_{\varepsilon}^{ \pm}\left(x, x^{\prime} ; m^{2}\right) d m^{2}\right)_{\varepsilon} .
$$

Interchanging the order of integration and taking the limit $x \rightarrow x^{\prime}$ one obtains

$$
\left(W_{\varepsilon}^{ \pm}\right)_{\varepsilon}=\frac{i}{2}\left(\int_{m^{2}}^{\infty} d m^{2} d^{n} x\left[-g^{ \pm}(x, \varepsilon)\right]^{\frac{1}{2}} G_{\varepsilon}^{ \pm}\left(x, x ; m^{2}\right)\right)_{\varepsilon} .
$$

Colombeau quantity $\left(W_{\varepsilon}^{ \pm}\right)_{\varepsilon}$ is called as the one-loop effective action. In the case of fermion effective actions, there would be a remaining trace over spinorial indices. From Eq. (6.39) we may define an effective Lagrangian density $\left(L_{\varepsilon ; \text { eff }}^{ \pm}(x)\right)_{\varepsilon}$ by

$$
\left(W_{\varepsilon}^{ \pm}\right)_{\varepsilon}=\left(\int d^{n} x\left[-g^{ \pm}(x, \varepsilon)\right]^{\frac{1}{2}} L_{\varepsilon ; \text { eff }}^{ \pm}(x)\right)_{\varepsilon},
$$

whence one gets 


$$
\left(L_{\varepsilon}^{ \pm}(x)\right)_{\varepsilon}=\left[-g^{ \pm}(x, \varepsilon)\right]^{\frac{1}{2}} \mathscr{L}_{\varepsilon ; \mathrm{eff}}^{ \pm}(x)=\frac{i}{2}\left(\lim _{x \rightarrow x^{\prime}} \int_{m^{2}}^{\infty} d m^{2} G_{\varepsilon}^{ \pm}\left(x, x^{\prime} ; m^{2}\right)\right)_{\varepsilon} .
$$

\subsection{Stress-tensor renormalization}

Note that $\left(L_{\varepsilon}^{ \pm}(x)\right)_{\varepsilon}$ diverges at the lower end of the $s$ integral because the $\sigma / 2 s$ damping factor in the exponent vanishes in the limit $x \rightarrow x^{\prime}$. (Convergence at the upper end is guaranteed by the $-i \epsilon$ that is implicitly added to $m^{2}$ in the De Witt-Schwinger representation of $\left(L_{\varepsilon}^{ \pm}(x)\right)_{\varepsilon}$. In four dimensions, the potentially divergent terms in the DeWitt- Schwinger expansion of $\left(L_{\varepsilon}^{ \pm}(x)\right)_{\varepsilon}$ are

$$
\left\{\begin{array}{c}
\left(L_{\varepsilon ; d i v}^{ \pm}(x)\right)_{\varepsilon}= \\
-\left(32 \pi^{2}\right)^{-1}\left(\operatorname { l i m } _ { x \rightarrow x ^ { \prime } } \left[\left(\Delta_{ \pm}^{1 / 2}\left(x, x^{\prime} ; \varepsilon\right)\right)_{\varepsilon} \int_{0}^{\infty} \frac{d s}{s^{3}} \exp \left[-i m^{2} s+\frac{\sigma\left(x, x^{\prime}\right)}{2 i s}\right] \times\right.\right. \\
\left.\times\left[a_{0}^{ \pm}\left(x, x^{\prime} ; \varepsilon\right)+i s a_{1}^{ \pm}\left(x, x^{\prime} ; \varepsilon\right)+(i s)^{2} a_{2}^{ \pm}\left(x, x^{\prime} ; \varepsilon\right)\right]\right)_{\varepsilon},
\end{array}\right.
$$

where the coefficients $a_{0}^{ \pm}, a_{1}^{ \pm}$and $a_{2}^{ \pm}$are given by Eq. (6.9)-Eq. (6.10). The remaining terms in this asymptotic expansion, involving $a_{3}^{ \pm}$and higher, are finite in the limit $x \rightarrow x^{\prime}$.

Let us determine now the precise form of the geometrical $\left(L_{\varepsilon ; d i v}^{ \pm}(x)\right)_{\varepsilon}$ terms, to compare them with the conventional gravitational Lagrangian that appears in (2.38). This is a delicate matter because (6.48) is, of course, infinite. What we require is to display the divergent terms in the form $\infty \times$ [geometrical object ]. This can be done in a variety of ways. For example, in $n$ dimensions, the asymptotic (adiabatic) expansion of $\left(L_{\varepsilon ; \text { eff }}^{ \pm}(x)\right)_{\varepsilon}$ is

$$
\left\{\begin{array}{c}
\left(L_{\varepsilon ; \text { eff }}^{ \pm}(x)\right)_{\varepsilon} \asymp \\
2^{-1}(4 \pi)^{-n / 2}\left(\lim _{x \rightarrow x^{\prime}}\left[\left(\Delta_{ \pm}^{1 / 2}\left(x, x^{\prime} ; \varepsilon\right)\right)_{\varepsilon}\right] \sum_{j=0}^{\infty} a_{j}\left(x, x^{\prime} ; \varepsilon\right) \times\right. \\
\left.\times \int_{0}^{\infty} i d s(i s)^{j-1-n / 2} \exp \left[-i m^{2} s+\frac{\sigma\left(x, x^{\prime}\right)}{2 i s}\right]\right)_{\varepsilon}
\end{array}\right.
$$

of which the first $n / 2+1$ terms are divergent as $\sigma \rightarrow 0$. If $n$ is treated as a variable which can be analytically continued throughout the complex plane, then we may take the $x \rightarrow x^{\prime}$ limit

$$
\left\{\begin{array}{c}
\left(L_{\varepsilon ; \text { eff }}^{ \pm}(x)\right)_{\varepsilon} \asymp 2^{-1}(4 \pi)^{-n / 2}\left(\sum_{j=0}^{\infty} a_{j}(x ; \varepsilon) \int_{0}^{\infty} i d s(i s)^{j-1-n / 2} \exp \left[-i m^{2} s\right]\right)_{\varepsilon}= \\
2^{-1}(4 \pi)^{-n / 2} \sum_{j=0}^{\infty} a_{j}(x ; \varepsilon)\left(m^{2}\right)^{n / 2-j} \Gamma\left(j-\frac{n}{2}\right) \\
a_{j}(x ; \varepsilon)=a_{j}(x, x ; \varepsilon) .
\end{array}\right.
$$

From Eq. (6.44) it follows we shall wish to retain the units of $L_{\varepsilon ; \text { eff }}^{ \pm}(x)$ as (length) ${ }^{-4}$, even when $n \neq 4$. It is therefore necessary to introduce an arbitrary mass scale $\mu$ and to rewrite Eq.(6.44) as

$$
\left(L_{\varepsilon ; \text { eff }}^{ \pm}(x)\right)_{\varepsilon} \asymp 2^{-1}(4 \pi)^{-n / 2}\left(\frac{m}{\mu}\right)^{n-4}\left(\sum_{j=0}^{\infty} a_{j}(x ; \varepsilon)\left(m^{2}\right)^{4-2 j} \Gamma\left(j-\frac{n}{2}\right)\right)_{\varepsilon} .
$$

If $n \rightarrow 4$, the first three terms of Eq.(6.45) diverge because of poles in the $\Gamma$ - functions: 


$$
\left\{\begin{array}{c}
\Gamma\left(-\frac{n}{4}\right)=\frac{4}{n(n-2)}\left(\frac{2}{4-n}-\gamma\right)+O(n-4), \\
\Gamma\left(1-\frac{n}{2}\right)=\frac{4}{(2-n)}\left(\frac{2}{4-n}-\gamma\right)+O(n-4), \\
\Gamma\left(2-\frac{n}{2}\right)=\frac{2}{4-n}-\gamma+O(n-4) .
\end{array}\right.
$$

Denoting these first three terms by $\left(L_{\varepsilon ; \mathbf{d i v}}^{ \pm}(x)\right)_{\varepsilon}$, we have

$$
\begin{gathered}
\left(L_{\varepsilon ; \operatorname{div}}^{ \pm}(x)\right)_{\varepsilon}=(4 \pi)^{-n / 2} \\
\left\{\frac{1}{n-4}+\frac{1}{2}\left[\gamma+\ln \left(\frac{m^{2}}{\mu^{2}}\right)\right]\right\}\left(\left[\frac{4 m^{4} a_{0}(x ; \varepsilon)}{n(n-2)}-\frac{2 m^{2} a_{1}(x ; \varepsilon)}{n-2}+a_{2}(x ; \varepsilon)\right]\right)_{\varepsilon} .
\end{gathered}
$$

The functions $a_{0}(x ; \varepsilon), a_{1}(x ; \varepsilon)$ and $a_{2}(x ; \varepsilon)$ are given by taking the coincidence limits of $(6.9)-(6.10)$

$$
\left\{\begin{array}{c}
\left(a_{0}^{ \pm}(x ; \varepsilon)\right)_{\varepsilon}=1, \\
\left(a_{1}^{ \pm}(x ; \varepsilon)\right)_{\varepsilon}=\left(\frac{1}{6}-\xi\right)\left(\mathbf{R}^{ \pm}(\varepsilon)\right)_{\varepsilon}, \\
\left(a_{2}^{ \pm}(x ; \varepsilon)\right)_{\varepsilon}=\frac{1}{180}\left(\mathbf{R}_{\alpha \beta \gamma \delta}^{ \pm}(x, \varepsilon) \mathbf{R}^{ \pm \alpha \beta \gamma \delta}(x, \varepsilon)\right)_{\varepsilon}-\frac{1}{180}\left(\mathbf{R}^{ \pm \alpha \beta}(x, \varepsilon) \mathbf{R}_{\alpha \beta}^{ \pm}(x, \varepsilon)\right)_{\varepsilon}- \\
-\frac{1}{6}\left(\frac{1}{5}-\xi\right)\left(\square \mathbf{R}^{ \pm}(x, \varepsilon)\right)_{\varepsilon}+\frac{1}{2}\left(\frac{1}{6}-\xi\right)\left(\mathbf{R}^{ \pm 2}(x, \varepsilon)\right)_{\varepsilon} .
\end{array}\right.
$$

Finally one obtains

$$
\left(L_{\varepsilon ; \text { ren }}^{ \pm}(x)\right)_{\varepsilon} \asymp-\frac{1}{64 \pi^{2}}\left(\int_{0}^{\infty} i d s \ln (i s) \frac{\partial^{3}}{\partial(i s)^{3}}\left[\mathcal{F}_{\varepsilon}^{ \pm}(x, x ; i s) e^{-i s m^{2}}\right]\right)_{\varepsilon} .
$$

Special interest attaches to field theories in distributional spacetime in which the classical action $\left(\mathbf{S}_{\varepsilon}\right)_{\varepsilon}$ is invariant under distributional conformal transformations,i.e.,

$$
\left(g_{\mu \nu}(x, \varepsilon)\right)_{\varepsilon} \rightarrow\left(\Omega_{\varepsilon}^{2}(x) g_{\mu \nu}(x, \varepsilon)\right)_{\varepsilon} \triangleq\left(\bar{g}_{\mu \nu}^{ \pm}(x, \varepsilon)\right)_{\varepsilon} .
$$

From the definitions one has

$$
\left(\mathbf{S}_{\varepsilon}\left[\bar{g}_{\mu \nu}^{ \pm}(x, \varepsilon)\right]\right)_{\varepsilon}=\left(\mathbf{S}_{\varepsilon}\left[g_{\mu \nu}^{ \pm}(x, \varepsilon)\right]\right)_{\varepsilon}+\left(\int d^{n} x\left(\frac{\delta \mathbf{S}_{\varepsilon}\left[\bar{g}_{\mu \nu}^{ \pm}(x, \varepsilon)\right]}{\delta \bar{g}^{ \pm \rho \sigma}(x, \varepsilon)}\right) \delta \bar{g}^{ \pm \rho \sigma}(x, \varepsilon)\right)_{\varepsilon}
$$

From Eq.(6.51) one obtains

$$
T_{\rho}^{ \pm} \quad \rho\left[g_{\mu \nu}^{ \pm}(x, \varepsilon), \varepsilon\right]=-\left.\left(\frac{\Omega_{\varepsilon}^{2}(x)}{[-g(x, \varepsilon)]^{\frac{1}{2}}} \frac{\delta \mathbf{S}_{\varepsilon}\left[\bar{g}_{\mu \nu}^{ \pm}(x, \varepsilon)\right]}{\delta \Omega_{\varepsilon}(x)}\right)_{\varepsilon}\right|_{\Omega_{\varepsilon}=1},
$$

and it is clear that if the classical action is invariant under the conformal transformations (6.50), then the classical stress-tensor is traceless. Because conformal transformations are essentially a rescaling of lengths at each spacetime point $x$, the presence of a mass and hence a fixed length scale in the theory will always break the conformal invariance. Therefore we are led to the massless limit of the regularization and renormalization procedures used in the previous section. Although all the higher order $(j>2)$ terms in the DeWitt-Schwinger expansion of the effective Lagrangian (6.45) are infrared divergent at $n=4$ as $m \rightarrow 0$, we can still use this expansion to yield the ultraviolet divergent terms arising from $j==0,1$, and 2 in the four-dimensional case. We may put $m=0$ immediately in the $j=0$ and 1 terms in the expansion, because they are of positive power for $n \sim 4$. These terms therefore vanish. The only nonvanishing potentially ultraviolet divergent term 
is therefore $j=2$ :

$$
2^{-1}(4 \pi)^{-n / 2}\left(\frac{m}{\mu}\right)^{n-4} a_{2}(x, \varepsilon) \Gamma\left(2-\frac{n}{2}\right),
$$

which must be handled carefully. Substituting for $a_{2}(x)$ with $\xi=\xi(n)$ from (6.48), and rearranging terms, we may write the divergent term in the effective action arising from (6.53) as follows

$$
\begin{gathered}
\left(W_{\varepsilon, \text { div }}^{ \pm}\right)_{\varepsilon}=2^{-1}(4 \pi)^{-n / 2}\left(\frac{m}{\mu}\right)^{n-4} \Gamma\left(2-\frac{n}{2}\right)\left(\int d^{n} x\left[-g^{ \pm}(x, \varepsilon)\right]^{\frac{1}{2}} a_{2}(x, \varepsilon)\right)_{\varepsilon}= \\
2^{-1}(4 \pi)^{-n / 2}\left(\frac{m}{\mu}\right)^{n-4} \Gamma\left(2-\frac{n}{2}\right)\left(\int d^{n} x\left[-g^{ \pm}(x, \varepsilon)\right]^{\frac{1}{2}}\left[\alpha \digamma_{\varepsilon}^{ \pm}(x)+\beta G_{\varepsilon}^{ \pm}(x)\right]\right)_{\varepsilon}+O(n-4),
\end{gathered}
$$

where

$$
\begin{gathered}
\left(\digamma_{\varepsilon}(x)\right)_{\varepsilon}=\left(\mathbf{R}^{ \pm \alpha \beta \gamma \delta}(x, \varepsilon) \mathbf{R}_{\alpha \beta \gamma \delta}^{ \pm}(x, \varepsilon)\right)_{\varepsilon}-2\left(\mathbf{R}^{ \pm \alpha \beta}(x, \varepsilon) \mathbf{R}_{\alpha \beta}^{ \pm}(x, \varepsilon)\right)_{\varepsilon}+\frac{1}{3}\left(\mathbf{R}^{ \pm 2}(x, \varepsilon)\right)_{\varepsilon} \\
\left(G_{\varepsilon}^{ \pm}(x)\right)_{\varepsilon}=\left(\mathbf{R}^{ \pm \alpha \beta \gamma \delta}(x, \varepsilon) \mathbf{R}_{\alpha \beta \gamma \delta}^{ \pm}(x, \varepsilon)\right)_{\varepsilon}
\end{gathered}
$$

and

$$
\alpha=\frac{1}{120}, \beta=-\frac{1}{360}
$$

Finally one obtains

$$
\begin{gathered}
\left\langle T_{\mu}^{\mu}(x, \varepsilon)\right\rangle_{\text {ren }}=-\left(1 / 2880 \pi^{2}\right)\left[\alpha\left(\digamma_{\varepsilon}(x)-\frac{2}{3} \square \mathbf{R}^{ \pm}(x, \varepsilon)\right)_{\varepsilon}+\beta\left(G_{\varepsilon}^{ \pm}(x)\right)_{\varepsilon}\right]= \\
-\left(1 / 2880 \pi^{2}\right)\left[\left(\mathbf{R}_{\alpha \beta \gamma \delta}^{ \pm}(x, \varepsilon) \mathbf{R}^{ \pm \alpha \beta \gamma \delta}(x, \varepsilon)\right)_{\varepsilon}-\left(\mathbf{R}_{\alpha \beta}^{ \pm}(x, \varepsilon) \mathbf{R}^{ \pm \alpha \beta}(x, \varepsilon)\right)_{\varepsilon}-\square \mathbf{R}^{ \pm}(x, \varepsilon)\right] .
\end{gathered}
$$

Note that from Eq.(3.42) for $\mathrm{r} \rightarrow 2 m$ it follows that

$$
\left(\mathbf{R}^{\rho \sigma \mu \nu}(\varepsilon) \mathbf{R}_{\rho \sigma \mu \nu}(\varepsilon)\right)_{\epsilon} \asymp\left(\left[(r-2 m)^{2}+\varepsilon^{2}\right]^{-1}\right)_{\varepsilon}+4(2 m)^{4} .
$$

Thus for the case of the distributional Schwarzchild spacetime given by the distributional metric (3.40) using Eq. (6.57) and Eq. (6.58) for $r \rightarrow 2 m$ one obtains

$$
\left\langle T_{\mu}^{\mu}(x, \varepsilon)\right\rangle_{\text {ren }} \asymp-\left(2880 \pi^{2}\right)^{-1}\left[\left(\left[(r-2 m)^{2}+\varepsilon^{2}\right]^{-1}\right)_{\varepsilon}+4(2 m)^{4}\right] .
$$

This result in a good agreement with Eq.(5.14) - Eq. (5.16).

\section{Conclusions and Remarks}

This paper dealing with an extension of the Einstein field equations using apparatus of contemporary generalization of the classical Lorentzian geometry named in literature Colombeau distributional geometry, see for example [1], [2], [3], [4], [5], [6], [7]. The regularizations of singularities present in some solutions of the Einstein equations is an important part of this approach. Any singularities present in some solutions of the Einstein equations recognized only in the sense of Colombeau generalized functions [1], [2] and not classically.

In this paper essentially new class Colombeau solutions to Einstein field equations is obtained. We have shown that a succesfull approach for dealing with curvature tensor valued distribution is to first 
impose admisible the nondegeneracy conditions on the metric tensor, and then take its derivatives in the sense of classical distributions in $\mathcal{S}_{2 m}^{\prime}\left(\mathbb{R}^{3}\right)$ space.

The distributional meaning is then equivalent to the junction condition formalism. Afterwards, through appropiate limiting procedures, it is then possible to obtain well behaved distributional tensors with support on submanifolds of $d \leq 3$, as we have shown for the energy-momentum tensors associated with the Schwarzschild spacetimes. The above procedure provides us with what is expected on physical grounds. However, it should be mentioned that the use of new supergeneralized functions (supergeneralized Colombeau algebras $\widetilde{\mathcal{G}}\left(\mathbb{R}^{3}, \Sigma\right)$ ) in order to obtain superdistributional curvatures, may render a more rigorous setting for discussing situations like the ones considered in this paper.

The vacuum energy density of free scalar quantum field $\Phi$ with a distributional background spacetime also is considered. It has been widely believed that, except in very extreme situations, the influence of gravity on quantum fields should amount to just small, sub-dominant contributions. Here we argue that this belief is false by showing that there exist well-behaved spacetime evolutions where the vacuum energy density of free quantum fields is forced, by the very same background distributional spacetime such BHs, to become dominant over any classical energy density component. This semiclassical gravity effect finds its roots in the singular behavior of quantum fields on curved spacetimes. In particular we obtain that the vacuum fluctuations $\left\langle\Phi^{2}\right\rangle$ have a singular behavior on BHs horizon $r_{+}:\left\langle\Phi^{2}(r)\right\rangle^{\sim}\left|r-r_{+}\right|^{-2}$. We argue that this vacuum dominance may bear important astrophysical implications.

\section{Acknowledgement}

To reviewers provided important clarifications.

\section{Competing Interests}

The authors declare that no competing interests exist.

\section{References}

[1] Colombeau JF. New generalized functions and multiplication of distributions. North Holland, Amsterdam; 1984.

[2] Colombeau JF. Elementary introduction to new generalized functions. North Holland, Amsterdam; 1985.

[3] Vickers JA, Wilson JP. A nonlinear theory of tensor distributions. gr-qc/9807068.

[4] Vickers JA, Wilson JP. Invariance of the distributional curvature of the cone under smooth diffeomorphisms. Class. Quantum Grav. 1999;16:579-588.

[5] Vickers JA. Nonlinear generalized functions in general relativity. In: Grosser M, et al. Nonlinear theory of generalized functions, Chapman \& Hall/CRC Research Notes in Mathematics. (Eds.) Chapman \& Hall CRC, Boca Raton. 1999;401:275-290.

[6] Kunzinger M, Steinbauer R. Nonlinear distributional geometry. Acta Appl.Math.; 2001.

[7] Kunzinger M, Steinbauer R. Generalized pseudo-riemannian geometry; 2001. Preprint mathFA/0107057.

[8] Tolman RC. Relativity, thermodynamics and cosmology. Clarendon Press, Oxford;1962.

[9] Landau LD, Lifschitz EM. The classical theory of fields. Pergamon Press, Oxford; 1975. 
[10] Geroch R, Traschen J. Strings and other distributional sources in general relativity. Phys. Rev. D. 1987;36:1017-1031.

[11] Balasin H, Nachbagauer H. On the distributional nature of the energy-momentum tensor of a black hole or what curves the schwarzschild geometry? Class. Quant. Grav. 1993;10:22712278.

[12] Balasin H, Nachbagauer H. Distributional energy-momentum tensor of the kerr-newman space-time family. Class. Quant. Grav. 1994;11:1453-1461.

[13] Kawai T, Sakane E. Distributional energy-momentum densities of schwarzschild space-time. Prog. Theor. Phys. 1997;98:69-86.

[14] Pantoja N, Rago H. Energy-momentum tensor valued distributions for the schwarzschild and reissner-nordstr448m geometries; 1997. Preprint gr-qc/9710072.

[15] Pantoja N, Rago H. Distributional sources in general relativity: Two point-like examples revisited; 2000. Preprint gr-qc/0009053.

[16] Grosser M, et al. On the foundations of nonlinear generalized functions I, II. Mem. Am. Math. Soc. 2001;153:729.

[17] Grosser M, et al. A global theory of nonlinear generalized functions. Adv. Math. to appear; 2001.

[18] Choquet-Bruhat Y. General relativity and the einstein equations. OUP Oxford: Dec 4. Mathematics. 2008;816.

[19] Biswas T. Physical interpretation of coordinates for the schwarzschild metric. Available: http://arxiv.org/abs/0809.1452v1

[20] Heinzle JM, Steinbauer R. Remarks on the distributional schwarzschild geometry; 2001. Preprint gr-qc/0112047.

[21] Hajicek P. An Introduction to the relativistic theory of gravitation. Springer Science \& Business Media: Aug 26. Mathematics. 2008;280.

[22] Schwartz L. Sur l'impossibilit439 de la Multiplication des Distributions. C. R. Acad. Sci. Paris. 1954;239:847-848.

[23] Gelfand IM, Schilov GE. Generalized functions. Vol. I: Properties and Operations, Academic Press, New York, London; 1964.

[24] Parker P. Distributional geometry. J. Math. Phys. 1979;20:1423-1426.

[25] Debney G, Kerr R, Schild A. Solutions of the einstein and Einstein-maxwell equations. J. Math. Phys. 1969;10:1842-1854.

[26] Abramowitz M, Stegun IA, editors. Handbook of mathematical functions with formulas, graphs, and mathematical tables, 9th printing. Dover, New York; 1972.

[27] Bracewell R. Heaviside's unit step function. In: The fourier transform and its applications. 3rd ed. McGraw-Hill, New York. 1999;57-61.

[28] Israel W. Nuovo cimento, 44, 1; Israel, W. Nuovo Cimento. 1966-1967;48:463.

[29] Raju CK. J. Phys. A: Math. Gen. 1982;15:1785.

[30] Kawai T, Sakane E. Distributional energy-momentum densities of schwarzschild space-time; 1998. Preprint grqc/9707029.

[31] Muller T, Grave F. Catalogue of spacetimes, Institut fur Visualisierung und Interaktive Systeme.

Available:http://xxx.lanl.gov/abs/0904.4184v2, http://www.vis.uni-stuttgart.de/ muelleta/CoS/ 
[32] Foukzon J. Distributional schwarzschild geometry from non smooth regularization via horizon. British Journal of Mathematics Computer Science. SCIENCEDOMAIN international. 2015;11(1):1-28. Article no. BJMCS.16961. ISSN: 2231-0851. www.sciencedomain.org

DOI: 10.9734/BJMCS/2015/16961

Available:http://vixra.org/pdf/1505.0118v2.pdf 


\section{Appendix A}

Expressions for the Colombeau quantities

$(\mathbf{R}(\{\},(\epsilon)))_{\epsilon},\left(\mathbf{R}^{\mu \nu}(\{\},(\epsilon)) \mathbf{R}_{\mu \nu}(\{\},(\epsilon))\right)_{\epsilon}$ and $\left(\mathbf{R}^{\rho \sigma \mu \nu}(\{\},(\epsilon)) \mathbf{R}_{\rho \sigma \mu \nu}(\{\},(\epsilon))\right)_{\epsilon}$ in terms of $\left(A_{\epsilon}\right)_{\epsilon},\left(B_{\epsilon}\right)_{\epsilon}$, $\left(C_{\epsilon}\right)_{\epsilon}$ and $\left(D_{\epsilon}\right)_{\epsilon}, \epsilon \in(0,1]$.

Let us introduce now Colombeau generalized metric which has the form

$$
\left\{\begin{array}{c}
\left(d s^{2}\right)_{\epsilon}=-\left(A_{\epsilon}(r)\left(d x^{0}\right)^{2}\right)_{\epsilon}-2\left(D_{\epsilon}(r) d x^{0} d r\right)_{\epsilon}+\left(\left(B_{\epsilon}(r)+C_{\epsilon}(r)\right)(d r)^{2}\right)_{\epsilon} \\
+\left(B_{\epsilon}(r) r^{2}\left[(d \theta)^{2}+\sin ^{2} \theta(d \phi)^{2}\right]\right)_{\epsilon} .
\end{array}\right.
$$

The Colombeau scalars $(\mathbf{R}(\epsilon))_{\epsilon},\left(\mathbf{R}^{\mu \nu}(\epsilon) \mathbf{R}_{\mu \nu}(\epsilon)\right)_{\epsilon}$ and $\left(R^{\rho \sigma \mu \nu}(\epsilon) R_{\rho \sigma \mu \nu}(\epsilon)\right)_{\epsilon}$, in terms of Colombeau generalized functions $\left(A_{\epsilon}(r)\right)_{\epsilon},\left(B_{\epsilon}(r)\right)_{\epsilon},\left(C_{\epsilon}(r)\right)_{\epsilon},\left(D_{\epsilon}(r)\right)_{\epsilon}$ are expressed as

$$
\begin{aligned}
& (\mathbf{R}(\epsilon))_{\epsilon}=\left(\frac { A _ { \epsilon } } { \Delta _ { \epsilon } } \left[\frac{2}{r}\left(-2 \frac{A_{\epsilon}^{\prime}}{A_{\epsilon}}-3 \frac{B_{\epsilon}^{\prime}}{B_{\epsilon}}+\frac{\Delta_{\epsilon}^{\prime}}{\Delta_{\epsilon}}\right)+\frac{2}{r^{2}} \frac{A_{\epsilon} C_{\epsilon}+D_{\epsilon}^{2}}{A_{\epsilon} B_{\epsilon}}-\frac{A_{\epsilon}^{\prime \prime}}{A_{\epsilon}}-2 \frac{B_{\epsilon}^{\prime \prime}}{B_{\epsilon}}\right.\right. \\
& \left.\left.+\frac{1}{2}\left(\frac{B_{\epsilon}^{\prime}}{B_{\epsilon}}\right)^{2}-2 \frac{A_{\epsilon}^{\prime} B_{\epsilon}^{\prime}}{A_{\epsilon} B_{\epsilon}}+\left(\frac{1}{2} \frac{A_{\epsilon}^{\prime}}{A_{\epsilon}}+\frac{B_{\epsilon}^{\prime}}{B_{\epsilon}}\right) \frac{\Delta_{\epsilon}^{\prime}}{\Delta_{\epsilon}}\right]\right)_{\epsilon}, \\
& \left(\mathbf{R}^{\mu \nu}(\epsilon) \mathbf{R}_{\mu \nu}(\epsilon)\right)_{\epsilon}=\left(\frac{A_{\epsilon}^{2}}{\Delta_{\epsilon}^{2}}\left(\frac{1}{2} \frac{A_{\epsilon}^{\prime \prime}}{A_{\epsilon}}-\frac{1}{4} \frac{A_{\epsilon}^{\prime} \Delta_{\epsilon}^{\prime}}{A \Delta_{\epsilon}}+\frac{1}{2} \frac{A_{\epsilon}^{\prime} B_{\epsilon}^{\prime}}{A_{\epsilon} B_{\epsilon}}+\frac{1}{r} \frac{A_{\epsilon}^{\prime}}{A_{\epsilon}}\right)^{2}\right)_{\epsilon}+ \\
& +2\left(\frac { A _ { \epsilon } ^ { 2 } } { \Delta _ { \epsilon } ^ { 2 } } \left[\frac{1}{r}\left(\frac{1}{2} \frac{\Delta_{\epsilon}^{\prime}}{\Delta_{\epsilon}}-\frac{A_{\epsilon}^{\prime}}{A_{\epsilon}}-2 \frac{B_{\epsilon}^{\prime}}{B_{\epsilon}}\right)+\frac{1}{r^{2}} \frac{A_{\epsilon} C_{\epsilon}+D_{\epsilon}^{2}}{A_{\epsilon} B_{\epsilon}}-\frac{1}{2} \frac{A_{\epsilon}^{\prime} B_{\epsilon}^{\prime}}{A_{\epsilon} B_{\epsilon}}-\right.\right. \\
& \left.\left.-\frac{1}{2} \frac{B_{\epsilon}^{\prime \prime}}{B_{\epsilon}}+\frac{1}{4} \frac{B_{\epsilon}^{\prime} \Delta_{\epsilon}^{\prime}}{B_{\epsilon} \Delta_{\epsilon}}\right]^{2}\right)_{\epsilon}+ \\
& \left(\frac { A _ { \epsilon } ^ { 2 } } { \Delta _ { \epsilon } ^ { 2 } } \left[\frac{1}{2} \frac{A_{\epsilon}^{\prime \prime}}{A_{\epsilon}}-\frac{1}{4} \frac{A_{\epsilon}^{\prime} \Delta_{\epsilon}^{\prime}}{A_{\epsilon} \Delta_{\epsilon}}+\frac{1}{2} \frac{A_{\epsilon}^{\prime} B_{\epsilon}^{\prime}}{A_{\epsilon} B_{\epsilon}}+\frac{B_{\epsilon}^{\prime \prime}}{B_{\epsilon}}-\frac{1}{2}\left(\frac{B_{\epsilon}^{\prime}}{B_{\epsilon}}\right)^{2}\right.\right. \\
& \left.\left.-\frac{1}{2} \frac{B_{\epsilon}^{\prime} \Delta_{\epsilon}^{\prime}}{B_{\epsilon} \Delta_{\epsilon}}+\frac{1}{r}\left(\frac{A_{\epsilon}^{\prime}}{A_{\epsilon}}-\frac{\Delta_{\epsilon}^{\prime}}{\Delta_{\epsilon}}+2 \frac{B_{\epsilon}^{\prime}}{B_{\epsilon}}\right)\right]^{2}\right)_{\epsilon}, \\
& \left(\mathbf{R}^{\rho \sigma \mu \nu}(\epsilon) \mathbf{R}_{\rho \sigma \mu \nu}(\epsilon)\right)_{\epsilon}= \\
& \left(\frac{A_{\epsilon}^{2}}{\Delta_{\epsilon}^{2}}\left(\frac{A_{\epsilon}^{\prime \prime}}{A_{\epsilon}}-\frac{1}{2} \frac{A_{\epsilon}^{\prime} \Delta_{\epsilon}^{\prime}}{A_{\epsilon} \Delta_{\epsilon}}\right)^{2}+2 \frac{A_{\epsilon}^{2}}{\Delta_{\epsilon}^{2}}\left(\frac{1}{r} \frac{A_{\epsilon}^{\prime}}{A_{\epsilon}}+\frac{1}{2} \frac{A_{\epsilon}^{\prime} B_{\epsilon}^{\prime}}{A_{\epsilon} B_{\epsilon}}\right)^{2}\right. \\
& +4 \frac{A_{\epsilon}^{2}}{\Delta_{\epsilon}^{2}}\left[\frac{1}{r} \frac{B_{\epsilon}^{\prime}}{B_{\epsilon}}-\frac{1}{r^{2}} \frac{A_{\epsilon} C_{\epsilon}+D_{\epsilon}^{2}}{A_{\epsilon} B_{\epsilon}}+\frac{1}{4}\left(\frac{B_{\epsilon}^{\prime}}{B_{\epsilon}}\right)^{2}\right]^{2}+ \\
& +2 \frac{A^{2}}{\Delta_{\epsilon}^{2}}\left[\frac{1}{r}\left(\frac{A_{\epsilon}^{\prime}}{A_{\epsilon}}+2 \frac{B_{\epsilon}^{\prime}}{B_{\epsilon}}-\frac{\Delta_{\epsilon}^{\prime}}{\Delta_{\epsilon}}\right)+\frac{1}{2} \frac{A_{\epsilon}^{\prime} B_{\epsilon}^{\prime}}{A_{\epsilon} B_{\epsilon}}+\frac{B_{\epsilon}^{\prime \prime}}{B_{\epsilon}}\right. \\
& \left.\left.-\frac{1}{2}\left(\frac{B_{\epsilon}^{\prime}}{B_{\epsilon}}\right)^{2}-\frac{1}{2} \frac{B_{\epsilon}^{\prime} \Delta_{\epsilon}^{\prime}}{B_{\epsilon} \Delta_{\epsilon}}\right]^{2}\right)_{\epsilon} \text {. }
\end{aligned}
$$

Here

$$
\left(\Delta_{\epsilon}\right)_{\epsilon}=\left(A_{\epsilon}(r)\left(B_{\epsilon}(r)+C_{\epsilon}(r)\right)\right)_{\epsilon}+\left(D_{\epsilon}^{2}(r)\right)_{\epsilon} .
$$

Assume that

$$
\left(\Delta_{\epsilon}(r)\right)_{\epsilon}=1,\left(B_{\epsilon}(r)\right)_{\epsilon}=1,\left(D_{\epsilon}(r)\right)_{\epsilon}=0 .
$$

From Eq. (A.2)-Eq. (A.4) one obtains 


$$
\begin{gathered}
(\mathbf{R}(\epsilon))_{\epsilon}=\left(-\frac{4 A_{\epsilon}^{\prime}}{r}+\frac{2 A_{\epsilon} C_{\epsilon}}{r^{2}}-A_{\epsilon}^{\prime \prime}\right)_{\epsilon} \\
\left(\mathbf{R}^{\mu \nu}(\epsilon) \mathbf{R}_{\mu \nu}(\epsilon)\right)_{\epsilon}= \\
\left(\left(\frac{1}{2} A_{\epsilon}^{\prime \prime}+\frac{1}{r} A_{\epsilon}^{\prime}\right)^{2}\right)_{\epsilon}+2\left(\left[-\frac{A_{\epsilon}^{\prime}}{r}+\frac{A_{\epsilon} C_{\epsilon}}{r^{2}}\right]^{2}\right)_{\epsilon}+\left(\left[\frac{1}{2} A_{\epsilon}^{\prime \prime}+\frac{1}{r} A_{\epsilon}^{\prime}\right]^{2}\right)_{\epsilon} \\
\left(\left(\mathbf{R}_{\epsilon}^{\prime \prime \prime}\right)^{2}+2\left(\frac{A_{\epsilon}^{\prime}}{r}\right)^{2}+4 \frac{\left(A_{\epsilon} C_{\epsilon}\right)^{2}}{r^{4}}+2\left(\frac{A_{\epsilon}^{\prime}}{r}\right)^{2}\right)_{\epsilon}
\end{gathered}
$$

(C)2016 Foukzon et al.; This is an Open Access article distributed under the terms of the Creative Commons Attribution License (http://creativecommons.org/licenses/by/4.0), which permits unrestricted use, distribution, and reproduction in any medium, provided the original work is properly cited.

Peer-review history:

The peer review history for this paper can be accessed here (Please copy paste the total link in your browser address bar)

http://sciencedomain.org/review-history/12977 